Noname manuscript No.

(will be inserted by the editor)

\title{
An executable formal semantics for UML-RT
}

\author{
Ernesto Posse - Juergen Dingel
}

the date of receipt and acceptance should be inserted later

\begin{abstract}
We propose a formal semantics for UMLRT, a UML profile for real-time and embedded systems. The formal semantics is given by mapping UML-RT models into a language called kiltera, a real-time extension of the $\pi$-calculus. Previous attempts to formalize the semantics of UML-RT have fallen short by considering only a very small subset of the language and providing fundamentally incomplete semantics based on incorrect assumptions, such as a one-to-one correspondence between "capsules" and threads. Our semantics is novel in several ways: 1) it deals with both state machine diagrams and capsule diagrams, 2) it deals with aspects of UML-RT which have not been formalized before, such as thread allocation, service provision points and service access points, 3) it supports an action language, and 4) the translation has been implemented in the form of a transformation from UML-RT models created with IBM's RSA-RTE tool, into kiltera code. To our knowledge, this is the most comprehensive formal semantics for UML-RT to date.
\end{abstract}

CR Subject Classification D.2.2 Design Tools and Techniques, D.3.1 Formal Definitions and Theory, D.3.3 Language Constructs and Features

\section{Contents}

1 Introduction . . . . . . . . . . . . . . . 1

2 Background ................... 5

3 The significance of thread allocation . . . . . . . . 11

4 State Machines . . . . . . . . . . . . . . . . 13

5 Capsules . . . . . . . . . . . . . . . 23 23

6 Related work . . . . . . . . . . . . . . 35

7 Concluding remarks $\ldots \ldots \ldots \ldots$

E. Posse $\cdot$ J. Dingel

Modelling and Analysis in Software Engineering, School of Computing, Queen's University, Kingston, Ontario, Canada.

\section{Introduction}

Real-Time Embedded systems (RTE) have become pervasive in avionics, telecommunications, manufacturing, traffic control, medical devices and automotive systems. Examples include anti-lock breaking systems in modern cars, telecom switches, aircraft flight-control systems and MRI machines.

RTE systems are typically characterized by being task-oriented, being part of a larger system, and often including safety-critical components. Being a part of a larger system implies that interaction is an essential aspect of an RTE system's behaviour. Furthermore, some of the components with which an RTE system interacts are physical components. This entails real-time constraints on the system's behaviour.

The term "real-time systems" encompasses a wide range of systems, languages, formalisms and even definitions of "real-time":

"There is no general consensus about the definition of both real-time and embedded terms." [41]

"[...] the term "real-time" covers a surprisingly diverse spectrum of systems, ranging from purely time-driven to purely event-driven systems, and from soft real- time systems to hard real-time systems." [54]

In this paper we focus on the event-driven, soft realtime side of the spectrum, in which it is not critical to meet every deadline every time. While timeliness is the defining characteristic of real-time systems, it is not the only concern. System architecture and good behaviour (e.g., safety, liveness, fairness) are fundamental concerns as well. 


\subsection{Model-Driven Development of RTE systems}

The development of RTE systems consists of, broadly speaking, several major activities: design and modelling, reasoning and analysis, and implementation and deployment. In design and modelling, engineers rely on languages and formalisms that provide adequate facilities to describe the systems to build, at the desired level of abstraction. Reasoning and analysis reduce risk by providing assurance that the system will meet its requirements. Implementation and deployment realize the system on specific platforms. These activities may be supported by automated tools. For instance, implementation may be aided by automatic code generation, and analysis by automatic model (formal) verification.

Model-Driven Development (MDD) is an approach to software development where models of a system and its components are the main artifact of the development process. In the MDD approach, models provide the basis for the activities outlined above. The engineer designs models, from which an implementation may be automatically generated. The MDD approach also facilitates analysis, as models, by definition, are abstractions of a system, and therefore likely to be simpler and easier to analyze than the final product.

An influential modelling language for the design of RTE systems which targets event-driven, soft real-time systems is the Real-Time Object Oriented Modeling language (ROOM) [56]. This language was later made into a UML profile called "UML-RT", introduced in [57, 54]. UML-RT is an industrial-strength language which has enjoyed considerable success with hundreds of largescale industrial projects and with users in a variety of sectors such as automotive, avionics and telecommunications, for which ROOM was originally designed. It has been supported by a number of commercial tools, including ObjectTime, Rational Rose RT, IBM's Rational Rose Technical Developer toolkit [22], and IBM Rational Software Architect Real Time Edition (IBM RSA-RTE) [23].

In order to be able to analyze models in a language, the language must have a well-defined semantics, otherwise, the meaning of models would be ambiguous, and the analysis results would be ad hoc, applicable only to, for example, specific models or specific implementations. Unfortunately, the semantics of UML-RT has only been defined informally. There have been some attempts at formalizing the semantics of UML-RT (e.g., $[61,5,4,7,16,31])$, but all of these attempts consider only limited subsets of the language, thus limiting the potential for analysis.

Our goal is to provide a comprehensive formal semantics for UML-RT, which can not only serve as a reference semantics but also supports both the execution and the analysis of models.

\subsection{UML-based modelling of RTE systems}

UML-RT, along with SDL [28] and Acme [17], heavily influenced the development of UML $2[42,44]$.

In addition to UML-RT, other UML profiles have been developed to account for timeliness and platform dependent issues, including the UML Schedulability, Performance and Time profile or UML SPTP [40] and its successor, the UML profile for Modeling and Analysis of Real-Time and Embedded Systems (MARTE) [41], both of which are OMG standards. Other unrelated UML profiles which are not OMG standards have been proposed as well, such as "Real-time UML", a.k.a. RTUML [11], and variants such as those in [3] and [38].

While there is some overlapping between UML-RT and UML SPTP and MARTE, there are significant differences. At the top-level, MARTE consists of three packages: the core package, the design modelling package and the analysis modelling package. The core package provides constructs to describe non-functional properties, time, generic resource modelling and allocation modelling. The design modelling package provides facilities for generic components, high-level application modelling and detailed resource modelling. The analysis package provides facilities for generic quantitative analysis modelling, schedulability analysis, and performance analysis. By contrast, UML-RT focuses on system architecture based on the notion of capsules (called actors in ROOM), ports, connectors, services and capsule structure diagrams, and event-driven behaviour described by state machines. MARTE's constructs and models are much more detailed than UML-RT's constructs and models. For instance, in MARTE, the basic unit of concurrent execution is called the RtUnit, defined in the HLAM package. This corresponds roughly to a capsule in UML-RT, but an RtUnit specifies many details such as memory size, message pool policies and waiting times, whereas a UML-RT capsule abstracts such details.

UML SPTP, MARTE and other similar profiles target the time-driven and hard real-time side of the spectrum of real-time systems, where the primary concern is timeliness, scheduling and platform dependent matters. According to Bran Selic, one of the main authors of ROOM, UML-RT and UML SPTP,

"MARTE deals with completely different aspects than UML-RT. MARTE addresses general issues related to real-time systems as they are usually implemented. Thus, it provides facilities 
for modeling time, resources, real-time operating systems (with their complex schedulers, lightweight threading systems, mutual exclusion facilities, etc.). It also provides support for modeling platforms of all kinds (including hardware), as well as facilities for doing real-time analyses, such as schedulability and queuing network analysis. ROOM/UML-RT, on the other hand, is a level of abstraction above that and does not deal with any of that." [55]

Hence, while MARTE is an appropriate formalism for the design and analysis of time-driven, resource intensive, and platform dependent, hard real-time systems, UML-RT may be better suited for soft-real time systems where such level of detail is a secondary concern.

Why should we be concerned with defining a semantics for UML-RT considering that the MARTE profile is available? We believe there are several important reasons: 1) UML-RT is in active industrial use, but there are no UML-RT development tools which provide formal analysis; a formal semantics would provide the basis for such analysis capabilities. 2) While the MARTE profile targets RTE systems, as argued above, UML-RT deals with related but different concerns and level of abstraction. 3) An executable formal semantics can also provide an analysis tool for simulation and validation of implementations. 4) Given that UML-RT played a central role in the definition of UML 2 and is fully aligned with it, a UML-RT formal semantics can help clarifying issues regarding the formal semantics of UML 2. 5) UML-RT has much in common with several architecture description languages such as AADL [53] and SysML [43], as well as a number of hardware description languages such as VHDL [25], Verilog [24], SystemVerilog [27], SystemC [26] and GDL [21], and a formal semantics for UML-RT can also suggest formal semantics for these languages, or can help elucidating their differences. 6) Formal semantics for foundational languages and calculi abound, but formal semantics for large, complex, industrial-strength languages are few. Our proposal can serve as a showcase for what such semantics can look like.

\subsection{UML-RT semantics by translation}

There are many approaches to formal semantics, such as denotational, operational, axiomatic, etc. Many such approaches do not yield an executable semantics. Even with operational semantics, which often takes the form of defining some form of transition system, considerable effort is required to obtain an executable artifact. The alternative is to define semantics by translating models to a language that already has a well-defined executable formal semantics.

In this paper we follow this approach. There are many possible choices for the target language such as CSP or the $\pi$-calculus, and we find such examples in the literature (see Section 6). However, these alternatives face many difficulties when formalizing a large, complex, high-level language such as UML-RT. Foundational calculi provide a solid basis for a formal semantics, but are limited in that the abstraction gap is often too wide. For example, these low-level calculi often lack higher level constructs to define complex data-types. Without such facilities in place, the task of defining a comprehensive formal semantics of a language such as UML-RT is almost unsurmountable. Hence we need a target language which is both executable and formal, as well as having higher level constructs to make the translation practical.

The target language we have chosen is called kiltera $[50,49,46,51]$. The main reasons for this choice are:

1. kiltera's semantic concepts have many similarities with those of UML-RT, providing a natural representation of UML-RT concepts,

2. kiltera has a well-defined formal semantics based on a real-time extension of the $\pi$-calculus [35], a process algebra for modelling and reasoning about concurrent, mobile systems, thus resting on a rich theory which provides a solid foundation for analysis, and

3. kiltera is a real high-level language with features to ease development and with a working implementation thus, providing the capability of executing models.

The goal of this article is to formally specify a translation from UML-RT models into kiltera. More concretely, we define a map $\mathcal{M} \llbracket \rrbracket:$ UMLRTC $\rightarrow$ KLT from UML-RT models to kiltera process terms, where UMLRTC is the set of valid UML-RT models and KLT is the set of valid kiltera terms.

UML-RT models describe both structural and behavioural aspects of a system. In the structural view, a model consists of a collection of interconnected components called capsules, which may have a behaviour and may themselves contain sub-capsules. The behaviour of capsules is specified by state machines. In this article we break down the translation into the behavioural and the structural parts. This is, we define a mapping for state machines and a mapping for capsule diagrams. Due to the modular nature of both UML-RT and kiltera, the two mappings are largely independent, therefore we only need to invoke the state machine translation without reference to its internals, in the capsule diagram mapping. This, in turn, allows for experimentation with alternative semantics, as it opens up the 
possibility of replacing state machines with some other formalism to specify behaviour.

\subsection{Shortcomings of existing UML-RT semantics}

As we mentioned above, there have been some attempts at formalizing the semantics of UML-RT (e.g., [61,5,4, $7,16,31]$, and also see Section 6).

The existing approaches to formalizing UML-RT fall short not only because of their limited scope and lack of support for syntactic features, but also because they provide fundamentally incomplete semantics. Those approaches will not include behaviours that are possible, and in some cases necessary, of UML-RT models. As a consequence, analysis of system behaviour may be incomplete or even erroneous. For example, as we will illustrate later, existing semantics are unable to distinguish between certain fair and unfair behaviours. The reason for this situation is that the existing approaches rely on incorrect assumptions.

Two of these fundamental aspects of the semantics of UML-RT which, with the exception of [31], have been ignored by every other attempt to formalize the language are the relation between capsules and threads, and the mechanism for communication between capsules. Apart from [31], all previous approaches make the incorrect assumptions that each capsule is executed as an independent (concurrent) thread, and that communication between them is direct, thus relying on the communication mechanism of the formalism or language used to describe the semantics (e.g., CSP or LOTOS), or assuming specific message-passing policies (e.g., synchronous communication). But this is not the case: capsules can be assigned to the same thread, sharing the same event queue, and the basic mechanism for message delivery is asynchronous and handled by a controller process. This however, is not a mere implementation issue or optimization issue, for it is semantically meaningful: different thread assignments, and different delivery mechanisms can yield different behaviours, for the same UML-RT model. Hence, by ignoring these aspects, other approaches provide an incorrect semantics which can result in incorrect analysis of system behaviour. In this paper we address this specific issue by providing explicitly in our proposed semantics, the controller's role, and the assignment of capsules to threads.

As stated above, our goal is to obtain a comprehensive account of the UML-RT semantics. While this article fails to cover all elements of UML-RT in detail, we believe it goes well beyond previous attempts to do so, and given its extensible nature, we are increasing the coverage of UML-RT's many features.

\subsection{Correctness and validation}

One of the main questions regarding the definition of a formal semantics for a language is validation. How do we know that our semantics is correct? Since the language we are formalizing lacks a formal semantics, we cannot prove mathematically the correctness of our semantics. How, then, can we be assured of our semantics' validity? We have addressed this problem by: 1) careful study of existing documentation on UML-RT and ROOM, 2) experimentation with the de facto reference implementations of UML-RT, specifically with Rational RoseRT and IBM's RSA-RTE, 3) inspection of code generated by these tools and their run-time systems, 4) consulting with Bran Selic, one of the lead designers of ROOM and UML-RT, and 5) developing a full implementation of the mapping using IBM's RSA transformation tool. The implementation produces code which can be executed with kiltera's simulator, allowing for validation against the output produced by RoseRT and RSA RTE. Having an actual implementation of the semantics also differentiates our work from previous attempts.

Just like UML, UML-RT has several semantic variation points, where, intentionally or unintentionally, the precise semantics is unspecified. Our definition is intended to be as close as possible to UML-RT as implemented by IBM's RSA-RTE. Nevertheless, some aspects can be considered to be implementation-specific and not mandatory for UML-RT models. In this paper we will mark such semantic variation points as SVP \# and the alternatives are proposed in the appendix.

Paper organization This article is organized as follows: In Section 2 we present background on UML-RT and kiltera. In Section 3 we present a motivating example that shows how thread allocation is essential to the semantics of UML-RT. Section 4 deals with state machines. In Subsection 4.1 we present a formal syntax for state machines, and the translation into kiltera is presented in Subsection 4.2. Section 5 addresses capsule diagrams. In Subsection 5.1 we present a formal syntax for capsule diagrams, and their translation is presented in Subsection 5.2. In Section 6 we discuss some related work and finally Section 7 concludes.

In the presentation of the formal translation of both state machines (Subsection 4.2) and capsule diagrams (Subsection 5.2), for each formal definition we proceed by first informally providing an overview of the concept being defined, and then we present the actual formal definition followed by a detailed explanation of the definition, and in the most important cases, an illustrative example. 


\section{Background}

\subsection{UML-RT}

In this section we describe informally the main concepts of UML-RT, in particular we describe the notions of capsules and structure diagrams in Subsection 2.1.1 and State Machines in Subsection 2.1.2. While UMLRT covers other types of UML diagrams, we focus only on these two, as they are the most important for UMLRT modelling. For more information on UML-RT we refer the reader to $[54,57,56]$. The official account of the UML can be found in $[42,44]$.

\subsubsection{Structure diagrams: capsules}

UML-RT allows modelling a system's structure through structure diagrams, also called capsule diagrams. Figure 1 shows a typical UML-RT capsule diagram.

A capsule, as its name suggests, is a highly encapsulated active entity, which may have some behaviour specified via a state machine (see Subsection 2.1.2). Capsules may execute concurrently with other capsules and communicate with them only by sending and receiving signals through ports $\left(p_{1}, p_{2}, \ldots, p_{13}\right.$ in Figure 1$)$. Ports in different capsules are linked by connectors (labelled $l_{1}, l_{2}, \ldots, l_{5}$ in Figure 1$)$. A connector links only two ports. Each port has a type specified with a protocol, which identifies signals that can be sent or received via the port. Communication may be asynchronous or synchronous. Capsules are organized hierarchically and each capsule may contain a number of instances of other capsules, called parts. External ports of these parts are connected (wired) statically or can be connected at runtime. Connected ports must implement the same protocol and be "compatible", i.e., the output (send) signals of one port must be the input (receive) signals of the other port and vice versa. In this case, one of the ports is said to be the base port and the other the conjugate port, e.g., $p_{6}$ and $p_{9}$ in Figure 1. A port marked with implements the conjugated version of a protocol, with the input and output signals inverted.

The set of ports of a capsule defines its interface. There are three kinds of ports: external end ports, external relay ports and internal ports. External end ports are ports linked to external capsules, and used directly by the capsule's state machine (if it has one) to either send or receive messages (e.g., port $p_{2}$ in Figure 1). External relay ports are ports directly connected to some sub-capsule (thus relay messages between some external capsule and some sub-capsule, e.g., ports $p_{1}$ and $p_{3}$ in Figure 1). Internal or protected ports, are used to communicate between the capsule's state machine and some sub-capsule (e.g., port $p_{4}$ in Figure 1).

Some ports such as $p_{12}$ and $p_{13}$ may be declared as unwired, but they may become connected or wired at run-time by explicit actions on the part of the capsules that own these ports. This is achieved when one of the ports is registered at runtime by its capsule as a service provision point or SPP for short, under a unique service name, and the other port is registered by its capsule as a service access point or SAP for short, under the same unique service name. When both ports are registered (which may be done asynchronously), a new connector links them. It is also possible to deregister ports and reregistering them, thus allowing a dynamic reconfiguration of the connections among capsules. SPPs and SAPs were not originally intended by the designers of UML-RT to be used for dynamic wiring between peer capsules [55] such as $C$ and $D$ in Figure 1. SPPs and SAPs were intended to be used as a mechanism for capsules to access services in the underlying layer or platform, in a multi-layer architecture. Nevertheless the language does not prohibit the dynamic wiring within the same layer, among peer capsules. Furthermore it is useful for modelling certain kinds of structural changes. For this reason, our mapping considers this operation as any other in the language.

A capsule is a class (in the OO sense) of components with ports. A capsule may have parts, which are instances of sub-capsules (and are attributes of the capsule's instance). A sub-capsule part may have one of three possible roles: fixed, optional or plug-in. A fixed sub-capsule is created (resp. destroyed) when its containing capsule is created (resp. destroyed) and is permanently attached to its containing capsule. An optional sub-capsule may be incarnated (i.e., created) or destroyed at a different time. Plug-in capsule roles are "placeholders" for capsules which can be filled and removed dynamically, and can be shared between different capsules. In Figure 1, $B$ is a fixed capsule, $C$ is an optional role, indicated by its light gray colour, and $D$ is a plug-in role, indicated by its blue colour.

Each capsule is assigned to a logical thread of control which in turn is assigned to some physical thread. A logical thread represents a conceptual concurrent thread of execution, while the physical thread is the actual runtime processing thread used by the underlying platform. That is, several capsules/logical threads can share the same real system thread. Each physical thread has a controller. A controller drives the execution of all capsules (logical threads) within a single physical thread. It contains the event pool for all events whose intended receiver is a capsule associated to the physical thread. It enforces run-to-completion semantics, this is, that 


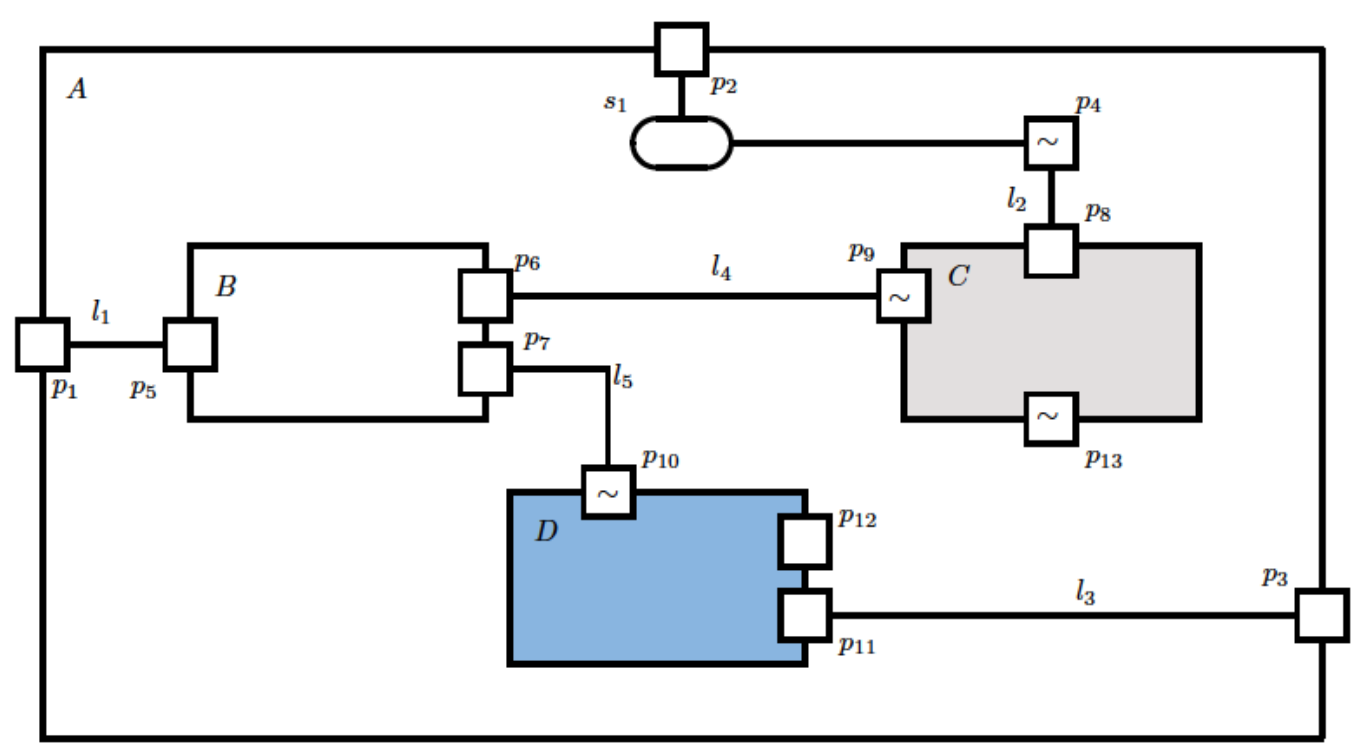

Fig. 1 A UML-RT structure (capsule) diagram

each state machine of each capsule it controls, processes each event fully, one at a time, before processing the next. This ensures that a capsule's state machine cannot be interrupted while processing an event. Subcapsules need not belong to the same logical or physical thread as their enclosing capsule.

Viewed as a UML profile, the structural elements of UML-RT correspond to UML 2 elements as follows: a capsule is a component, which is a structured classifier, and therefore an encapsulated classifier. Ports and connectors have the same name in UML 2, but in UML$\mathrm{RT}$ it is useful to distinguish between the internal, relay and end ports. SAPs and SPPs are particular kinds of ports.

\subsubsection{Behaviour diagrams: state machines}

The behaviour of a capsule is specified using UML-RT state machines $[54,57,56]$ which are similar to UML state machines $[42,44]$ but with some key differences. Figure 2 shows a typical UML-RT state machine diagram.

A UML-RT state machine has hierarchical states and guarded transitions, which are triggered by events received on ports. Each state declares its entry and exit actions and transitions have effects, so they can contain actions that are to be executed when the transition is fired. Just like in standard UML state machines, event handling in UML-RT state machines will follow a "runto-completion" semantics: a state machine will handle one and only one event at a time, and any transition chain enabled will be fully followed and its actions fully executed before the next event is handled.

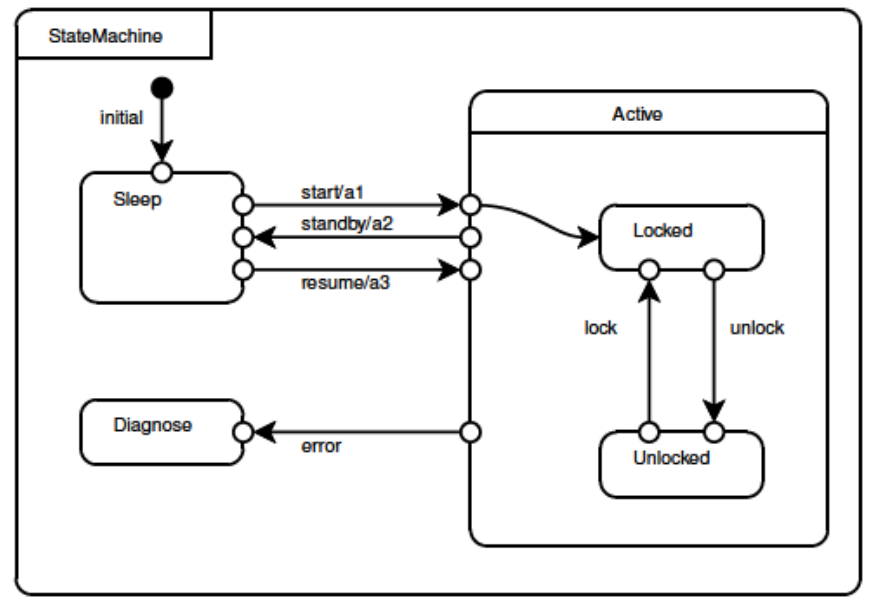

Fig. 2 A UML-RT state machine diagram.

However, there also are some important syntactic and semantic differences between UML-RT state machines and UML state machines:

1. UML-RT state machines cannot contain "and-states" (orthogonal regions). All states are "or-states". So, during execution a given UML-RT state machine can be only in at most one simple state.

2. Transitions in UML-RT state machines are not allowed to cross state boundaries and they may have explicit entry and exit points, here collectively called connection points (in UML terminology, entry and exit pseudo-states). Hence, to represent a boundarycrossing transition, it must be broken up into segments, where each segment links connection points, either at the same level of nesting, or between a state and an immediate sub-state. During execution, con- 
nected segments form a transition chain, which is executed as one step.

3. In UML-RT entry points are by default connected to deep history pseudo-states. Suppose a composite state $n$ is the target of a transition and that the associated entry point is not linked to a sub-state of $n$. If $n$ has not been previously visited and there is an initial transition pointing to the default state, then the initial transition is followed and the default state entered. If, however, $n$ has been visited previously, then the last sub-state visited in $n$ is entered. If it has not been visited and there is no initial transition, no sub-state is entered and the state machine remain "at the border" of $n$. This policy is applied recursively. Hence, entering a state can be interpreted as "resuming computation where it previously left off". In standard UML state machines, on the other hand, it is possible not to connect entry points to deep history pseudo-states, but to "shallow" history pseudo states, or to the boundary of the state, in which case an initial state is always entered, if an entry point is not explicitly connected to a sub-state. Since all states have deep-history semantics, we avoid the common notation of depicting deep history pseudo-states explicitly, to avoid clutter in the diagrams.

4. Actions may be related to concepts specific to UMLRT such as capsule operations. In particular an action may send an event through a port, create or destroy an optional capsule, import or deport a plug-in capsule, connect or disconnect unbound ports, and perform normal operations on objects.

5. UML-RT supports timing requirements using a special timing protocol and internal ports which implement this protocol. A capsule, which contains a port that implements the timing protocol, can schedule an event by sending a signal through this port. Scheduling can be a part of the entry or exit behavior of a state or as an action on a transition. After a specified amount of time, the capsule will receive a timeout event from the port which it can process as any other signal.

\subsubsection{Time}

In UML-RT, time is assumed to progress according to an external timing service (usually provided by the underlying platform). The timing service adheres to a timing protocol with a distinguished timeout signal and a period or a deadline. The timing service is accessible by UML-RT models through a standard port with the corresponding timing protocol, so time signals can be treated as any other signal. Since the timing service is external, it can proceed in any way that maintains time consistency, i.e., if two timers with timeout signals $t m o_{1}$ and $t m o_{2}$ are set up at the same time $t_{0}$ with timeouts $t_{1}>t_{0}$ and $t_{2}>t_{0}$ respectively, and such that $t_{1}<t_{2}$, then the timing service must guarantee that signal $t m o_{1}$ will be triggered before $t m o_{2}$. Besides this requirement, the semantics of UML-RT does not make any assumptions about the rate of progress of these clocks. Furthermore, since UML-RT is not concerned with performance or scheduling, it makes no assumptions about the duration of specific actions. We assume that individual actions in the underlying action language take a negligible amount of time with respect to the minimum time unit of the time services used. Furthermore, other activities such as entering or exiting a state, or relaying a message on a relay port, also take a negligible amount of time. In the case of asynchronous communication between capsules, the amount of time between the sending of a message and its reception and consumption is undetermined. If a capsule is in a state listening to a normal port and a timeout port, and the environment sends the message before the timeout, the language does not guarantee that the message will be consumed before the timeout signal arrives.

\section{2 kiltera}

Our approach to formalize the semantics of UML-RT is to use a process calculus or process algebra to describe the behaviour of a model. Process calculi or process algebras are mathematical formalisms for modelling and reasoning about concurrent systems in which a broad set of algebraic, logic and set theoretic techniques can be used to analyze system behaviour. Some of the best known process calculi are CCS [34], CSP [19], ACP [2] and the $\pi$-calculus [35].

kiltera $[50,49,46,51]$. is a language for modelling and simulating concurrent, interacting, real-time processes with support for mobility and distributed systems. It is directly based on the $\pi_{k l t}$ calculus [48] which is a realtime extension of the asynchronous $\pi$ calculus [35,20, $6]$, one of the best known variants of the $\pi$-calculus.

Just as in the $\pi$-calculus, the central notions are those of process and channel. A $\pi_{k l t}$ term represents a process or set of processes running concurrently. Processes interact by asynchronous message passing over channels. In kiltera we identify events and channels: triggering an event start is the same as sending a message over a channel named start, and listening to an event is the same as waiting for input on a channel. This event-oriented terminology is due to the fact that kiltera was originally designed in the context of modelling and simulation of discrete-event systems as treated in 
$[64,63]$. Just like the $\pi$ calculus, kiltera supports channel mobility: the ability to send channels (i.e., events) as part of messages. This allows the topology of the network to change dynamically.

In addition to communication primitives, $\pi_{k l t}$ extends the asynchronous $\pi$-calculus by introducing timing constructs (e.g., delaying the execution of a process, recording waiting times, and timeouts), primitive data values and data structures, pattern matching on input, nested process and function definitions with lexical scoping. These characteristics make it a high-level language, which facilitates our description of the semantics of UML-RT, while still having a formal semantics.

The formal semantics of $\pi_{k l t}$ is given in terms of a Plotkin-style structural operational semantics over timed-labelled transition systems. The meta theory of $\pi_{k l t}$ extends that of the $\pi$ calculus by a notion of timebounded equivalence and a notion of timed compositionality and an associated timed congruence which allow reasoning about timed processes.

We have developed an implementation of the language based on an abstract machine which has been proven sound with respect to $\pi_{k l t}$ 's operational semantics. The core simulation algorithm consists of event scheduling as known in discrete-event simulation [64]. The interpreter supports two modes: real-time and simulated time. In real-time mode, the wall-clock timing of events reflects delays and timeouts specified in the model, and thus the interpreter actually pauses during idle periods. In simulated time, execution proceeds according to a logical clock, and events are processed as soon as they are available, thus avoiding idling when the model specifies events far apart in time. Consequently, execution in simulated time mode is more efficient, while execution in real-time mode is more reflective of the timing constraints. Our interpreter is a prototype implemented in Python and does not use a realtime operating system; thus, in real-time mode, timing constraints are only approximated.

The full language also includes some constructs for distributed computing, allowing the execution of processes in logical sites. The simulator allows assigning kiltera sites to different physical machines, and distributed simulation is performed using a variation of the TimeWarp algorithm [29]. We have used kiltera in the modelling of complex systems such as automobile traffic simulation and cloud computing environments. kiltera has been used for teaching in graduate courses at McGill and Queen's universities. Our kiltera simulator is available for download at http://www.kiltera.org.

\subsubsection{Syntax}

To formally define the mapping we use the core of kiltera, the $\pi_{k l t}$ calculus, which has a mathematical notation suitable to describe the mapping.

Definition 1 (Syntax) The set of all $\pi_{k l t}$ process terms, denoted KLT is defined by the BNF in Figure 3. The same BNF defines the set Expr of expressions, ranged over by $E, E^{\prime}, \ldots$, the set Patts of patterns, ranged over by $R, R^{\prime}, \ldots$, and the set Defs of definitions, ranged over by $D, D^{\prime}, \ldots$ We usually write $a, b, c \ldots$ for channel/event names, $A, B, C, \ldots$ for process names, $x, y, z, \ldots$ for variables.

\subsubsection{Informal semantics}

We now describe informally the language's semantics. For a formal semantics of the language see [48]. For earlier versions of the semantics see $[50,47,46]$.

- Expressions $E$ are either constants (null represents the null constant), variables $(x)$, tuples of the form $\left\langle E_{1}, \ldots, E_{m}\right\rangle$ or function applications $f\left(E_{1}, \ldots, E_{m}\right)$. Patterns $R$ have the same syntax as expressions, except that they do not include function applications.

- The term stop represents the stopped process: it has no actions.

- The process done represents successful termination.

- The process $a ! E$ is a trigger; it triggers an event $a$ with the value of $E$. Alternatively, we can say that it sends the value of $E$ over a channel $a$. This is an asynchronous message sending, with no specific buffering policy mandated by the semantics. The expression $E$ is optional: $a$ ! is shorthand for $a$ !null.

- A process when $\left\{G_{1} \rightarrow P_{1}|\cdots| G_{n} \rightarrow P_{n}\right\}$ is a listener. Each $G_{i}$ is a guard of the form $a_{i}$ ? $R_{i} @ y_{i}$ where $a_{i}$ is an event/channel name, $R_{i}$ is a pattern, and $y_{i}$ is an optional variable. This process listens to all channels (or events) $a_{i}$, and when $a_{i}$ is triggered with a value $V$ that matches the pattern $R_{i}$, the corresponding process $P_{i}$ is executed with $y_{i}$ bound to the amount of time the listener waited, and the alternatives are discarded. Note that to enable an input guard it is not enough for the channel to be triggered: the message must match the guard's pattern as well. Pattern-matching of inputs means that the input value must have the same "shape" as the pattern, and if successful, the free names in the pattern are bound to the corresponding values of the input. For example, the value $\langle 3$, true, 7$\rangle$ matches the pattern $\langle 3, x, y\rangle$ with the resulting binding $\{$ true $/ x, 7 / y\}$. The scope of these bindings is the corresponding $P_{i}$. . The suffixes $R_{i}$ and $@ y_{i}$ are optional: $a$ ? $\rightarrow P$ is 


\begin{tabular}{|c|c|c|c|}
\hline \multirow[t]{12}{*}{$P$} & $::=$ & stop & Stopped process \\
\hline & | & done & Successful termination \\
\hline & | & $a ! E$ & Trigger/Output \\
\hline & | & when $\left\{G_{1} \rightarrow P_{1}|\cdots| G_{n} \rightarrow P_{n}\right\}$ & Listener/Input \\
\hline & | & new $a_{1}, \ldots, a_{n}$ in $P$ & New/Hide \\
\hline & | & if $E$ then $P_{1}$ else $P_{2}$ & Conditional \\
\hline & | & wait $E \rightarrow P$ & Delay \\
\hline & | & $A\left(E_{1}, \ldots, E_{n}\right)$ & Instantiation/Call \\
\hline & | & $\operatorname{def}\left\{D_{1} ; \ldots ; D_{n}\right\}$ in $P$ & Local definitions \\
\hline & | & $P_{1} \| P_{2}$ & Parallel composition \\
\hline & | & $P_{1} ; P_{2}$ & Sequential composition \\
\hline & | & $x:=E$ & Assignment \\
\hline G & $::=$ & $a ? R @ y$ & Listener/input guard \\
\hline$D$ & $::=$ & $\operatorname{proc} A\left(x_{1}, \ldots, x_{n}\right)=P$ & Process definition \\
\hline & | & func $f\left(x_{1}, \ldots, x_{n}\right)=E$ & Function definition \\
\hline & | & $\operatorname{var} x=E$ & Variable definition \\
\hline$E$ & $::=$ & \begin{tabular}{c|c|c|c|} 
null $\mid r$ & true $\mid$ false \\
$\left\langle E_{1}, \ldots, E_{m}\right\rangle \mid$ & $f\left(E_{1}, \ldots, E_{m}\right)$
\end{tabular} & "s" | $x$ \\
\hline$R$ & $::=$ & $\begin{array}{l}\operatorname{null}|r| \text { true } \mid \text { false } \\
\left\langle R_{1}, \ldots, R_{m}\right\rangle\end{array}$ & "s" | $x$ \\
\hline
\end{tabular}

Fig. $3 \pi_{k l t}$ syntax

equivalent to $a ? x @ y \rightarrow P$ for some fresh names $x$ and $y$.

- The process new $a_{1}, \ldots, a_{n}$ in $P$ hides the names $a_{i}$ from the environment, so that they are private to $P$. Alternatively, new $a_{1}, \ldots, a_{n}$ in $P$ can be seen as the creation of new names, i.e., , new events or channels, whose scope is $P$.

- The process wait $E \rightarrow P$ is a delay: it delays the execution of process $P$ by an amount of time equal to the value of the expression $E$. The value of $E$ is expected to be a non-negative real number. If the value of $E$ is negative, wait $E \rightarrow P$ cannot perform any action. Similarly, terms with undefined values (e.g., , wait $1 / 0 \rightarrow P$ ) or with incorrectly typed expressions $(e . g .$, , wait true $\rightarrow P$ ) cause the process to stop. Since the language is untyped we do not enforce these constraints statically.

- The process if $E$ then $P_{1}$ else $P_{2}$ is a conditional with the standard meaning. if $E$ then $P$ is shorthand for if $E$ then $P$ else done.

- The process $P_{1} \| P_{2}$ is the parallel composition of $P_{1}$ and $P_{2}$. We also allow an indexed parallel composition, written $\prod_{i \in I} P_{i}$ to stand for $P_{1}\left\|P_{2}\right\| \cdots \| P_{n}$ for some index set $I=\{1,2, \ldots, n\}$.

- The term $P_{1} ; P_{2}$ is the sequential composition of $P_{1}$ and $P_{2}$.

- The term def $\left\{D_{1} ; \ldots ; D_{n}\right\}$ in $P$ declares definitions $D_{i}$ and executes $P$. The scope of these definitions is the entire term (so they can be invoked in $P$ and in other definitions). Each $D_{i}$ can be either a process definition proc $A\left(x_{1}, \ldots, x_{n}\right)=P$, a function definition func $f\left(x_{1}, \ldots, x_{n}\right)=E$ or a local variable definition $\operatorname{var} x=E$.

- The term $x:=E$ assigns the value of $E$ to the local variable $x$.

- The process $A\left(E_{1}, \ldots, E_{n}\right)$ creates a new instance of a process defined by proc $A\left(x_{1}, \ldots, x_{n}\right)=P$, defined in some enclosing scope, where the ports or parameters $x_{1}, \ldots, x_{n}$ are substituted in the body $P$ by the values of expressions $E_{1}, \ldots, E_{n}$, which may be channel names.

\subsubsection{Some examples and usage patterns}

In order to give the reader some intuition about the semantics of $\pi_{k l t}$ we present some representative examples and common patterns.

Interaction The process $a ! \|$ when $\{a$ ? $\rightarrow P\}$ results in one interaction between the processes and then continues as done $\| P$ which is the same as just $P$.

Choice The term $a$ ! $\|$ when $\{a ? \rightarrow P \mid b ? \rightarrow Q\}$ reduces to $P$, while $b$ ! $\|$ when $\{a$ ? $\rightarrow P \mid b$ ? $\rightarrow Q\}$ reduces to $Q$. If the environment of a listener triggers more than one of the listener's guards, the choice is non-deterministic: 
$a !\|b !\|$ when $\{a ? \rightarrow P \mid b ? \rightarrow Q\}$ can reduce to either $b ! \| P$ or $a ! \| Q$.

Pattern matching For interaction to happen, data received must match the expected pattern: the process $a$ !"hi" \| when $\{a$ ?"hi" $\rightarrow P\}$ reduces to $P$. On the other hand, a! "hi" \| when $\{a$ ?"hey" $\rightarrow P\}$ does not result in an interaction because the data sent over $a$ ("hi") does not match the expected pattern ("hey"). Hence the two processes remain the same. If the pattern has variables, a successful communication results in substituting the corresponding variables by the received values: $a$ ! "hi" $\|$ when $\{a ? x \rightarrow P\}$ results in $P\{$ "hi" $/ x\}$, this is, substituting every free occurrence of $x$ in $P$ by "hi". The same holds for more complicated patterns: the term $a$ ! 〈"hi", 6$\rangle \|$ when $\{a$ ? $\langle$ "hi", $x\rangle \rightarrow P\}$ results in $P\{6 / x\}$.

Local channels The new construct introduces new names and restricts their scope. For example, in the term $a ! 1 \|$ new $a$ in $(a ! 2 \|$ when $\{a ? x \rightarrow P\})$ the $a$ in $a ! 1$ is different from the one in $a ! 2$. The whole term reduces to $a ! 1 \| P\{2 / x\}$.

Barriers and joining It is common for a process to wait for several other processes before continuing. This can be achieved with nested listeners: in (wait $3 \rightarrow a$ !) $\|$ $b$ ! $\|$ when $\{a$ ? $\rightarrow$ when $\{b$ ? $\rightarrow P\}\}$, process $P$ will begin only when both $a$ and $b$ have been triggered. This example also shows that the triggers are persistent, this is, the trigger $b$ ! is not lost if no other process is listening to $b$, and remains available until some process is ready to accept it. So the whole process waits 3 time units and becomes $a ! \| b$ ! $\|$ when $\{a$ ? $\rightarrow$ when $\{b ? \rightarrow P\}\}$ which then becomes $b$ ! $\|$ when $\{b$ ? $\rightarrow P\}$ which finally becomes $P$. This notion of nested listeners as barriers is so useful that we will write when $\{\langle a, b\rangle ? \rightarrow P\}$ as syntactic sugar for when $\{a$ ? $\rightarrow$ when $\{b ? \rightarrow P\}\}$. The sequential composition operator is also useful for joining processes: in $(P \| Q) ; R$ process $R$ will start only after both $P$ and $Q$ are done.

Process definitions Process definitions allow us to encapsulate processes, giving them a specific interface and be reused in the scope of their definition. For example, $\operatorname{def}\{\operatorname{proc} P(x)=x ! ; \operatorname{proc} C(y)=$ when $\{y$ ? $\rightarrow Q\}\}$ in new $a$ in $(P(a) \| C(a))$ results in the same process as the term new $a$ in $(a$ ! \| when $\{a$ ? $\rightarrow Q\})$. The parameters of a process definition can be thought of as its interface, its ports, so when we invoke the process definition we can visualize it as creating an instance of the process and "hooking up" channels to its ports; e.g., in $P(a)$ we are instantiating $P$ and hooking-up the local channel $a$ to the new instance's port $x$. Nevertheless, parameters are not required to be only channels or events, but they can be any value. This fact is used for example to keep track of additional state variables.

Recursion The body of a process can refer to itself, or even to other processes in the same definition group (or any enclosing process definitions). Recursion is used by a process to keep itself alive, and possibly change its connections by invoking itself with different parameters. For example consider the definition proc $A(x, y)=$ when $\{x ? z \rightarrow(y ! \| A(z, y))\}$. Then, executing $A(a, b) \|$ $a ! c$ will result in when $\{a ? z \rightarrow(b ! \| A(z, b))\} \| a ! c$ which will then reduce to $b$ ! $\| A(c, b)$.

Lexical scoping This applies to names introduced with new, names introduced with def and pattern variables. This is, the occurrence of a name $x$ always refers to the closest enclosing construct that declares it, e.g., in $\operatorname{proc} A(x, y, z)=$ when $\{x ? y \rightarrow$ new $z$ in $y !\langle x, z\rangle\}$, in the innermost term $y !\langle x, z\rangle, x$ refers to the first parameter of $A, y$ refers to the pattern in the listener's guard $x ? y$ (not $A$ 's second parameter) and $z$ refers to the one introduced by new $z$ (and not to $A$ 's third parameter).

Channel mobility Channels or events are first-class objects, so they can be included in messages: reducing $a ! b \|$ when $\{a ? x \rightarrow x ! c\}$ results in $b ! c$. This is allowed even for private or local names. For example the term when $\{a ? x \rightarrow x ! c\} \quad \|$ new $b$ in $(a ! b \| P)$ reduces to the term new $b$ in $(b ! c \| P)$. In this case, the right-hand subprocess sent a private channel $b$ to the left-hand subprocess via $a$. Hence the left-hand process evolves into $b ! c$ becoming aware of the private $b .{ }^{1}$

Asynchronous message passing As in the asynchronous $\pi$-calculus, asynchronous communication is modelled by syntactically restricting the output operator by not allowing it to have a continuation. In practice, however it is often desired to allow writing, e.g., $a ! 1 \rightarrow P$. This however is only syntactic sugar for $a ! 1 \| P$, as the process $P$ is free to continue without having to wait for the output $a ! 1$ to be consummated.

Message acknowledgment and response Since communication is asynchronous, when sending a message, the sender does not wait for the receiver to get and acknowledge the message, e.g., in $a$ ! "hi"; $Q$ process $Q$ can begin before any process receives the message sent over

\footnotetext{
${ }^{1}$ In the $\pi$-calculus literature this is known as scope extrusion as the lexical scope of the private name is effectively extended beyond its original scope.
} 
$a$. Nevertheless, we often wish to receive an acknowledgment or response from a receiver. A common way to do this in the $\pi$-calculus is to use channel mobility: create a local channel, say $r$ where the sender will expect the acknowledgment or response, send $r$ as part of the query and listen to $r$ before proceeding. The response message on $r$ may be empty to signal acknowledgement, or may include data, such as the answer to the query. This can also be seen as a simple way to encode synchronous message passing or remote procedure calls. The response channel needs to be local to remain private, avoiding interference from other processes. For example, the sender could be proc $S(q)=$ new $r$ in $(q ! r \|$ when $\{r ? x \rightarrow P\})$ and the receiver could be $\operatorname{proc} R(q)=$ when $\{q ? r \rightarrow(Q ; r$ ! "result" $)\}$. Thus, the sender sends a query on channel $q$ including its private channel $r$ where it will expect the response, and then listens to $r$. Once the response arrives, it proceeds as $P$. The receiver waits for a query on $q$ and when the query arrives it is expected to come with a response channel $r$. Then it proceeds to do some task $Q$ and when it is done, it sends the result on channel $r$. We use this pattern repeatedly throughout our translation.

Process names as parameters In process definitions, process invocations, expressions and patterns, we allow the names $x$ to be process and function names as well. This is an essential feature that allows us to write generic processes, for example: def $\{\operatorname{proc} A(x)=$ $x ! 1 ; \operatorname{proc} B(y, Z)=Z(y)\}$ in new $u$ in $B(u, A)$. In this example, the second parameter passed to $B$ is $A$, so executing $B(u, A)$ results in $A(u)$.

\section{Auxiliary functions}

While data structures such as lists and dictionaries (associative tables) are not primitive, they can be encoded in this language. It is outside the scope of this paper to provide such encodings, but for convenience we will assume the following functions as primitive:

- empty_list: the empty list constant,

- list_add(item, list): returns the list that has item as the first element and list as the remainder,

- list_pop(list): returns a pair 〈item, rem〉 where item was the first element of list and rem was the rest,

- list_del(item, list): returns the list without item,

- list_isempty $($ list $)$ : returns true if the list is empty, and false otherwise,

- empty_dict: the empty dictionary constant,

- dict_put (key, value, dict): returns a dictionary that adds the association $\langle k e y$, value $\rangle$ to the dictionary dict, if there was no pair with the given key, oth- erwise, it replaces the existing association $\langle k e y, v\rangle$ with the association $\langle k e y$, value $\rangle$,

- dict_get (key, dict) returns the value associated with the key in the dictionary dict, or null if the key has no associated value,

- dict_del(key, dict) returns the dictionary dict with any association $\langle k e y, v\rangle$ removed.

\subsection{Additional preliminaries}

Here we define some additional notation used throughout the paper.

We write $1 . . k$ for the set $\{1,2, \ldots, k\}$. Sequences will be enclosed in $\langle$ and $\rangle$. A sequence name will be denoted with an arrow on top, and its elements subscripted with their index, beginning from 1: $\tilde{x}=\left\langle x_{1}, x_{2}, x_{3}, \ldots\right\rangle$. A finite sequence $\left\langle a_{1}, \ldots, a_{k}\right\rangle$ will be abbreviated as $a_{1 . . k}$. The empty sequence is denoted \langle\rangle , or $\epsilon$. We will also use standard set operators for sequences, in particular we write $x \in \tilde{x}$ for membership of $x$ in the sequence $\tilde{x}$.

\section{The significance of thread allocation}

As suggested in the introduction, thread allocation is a fundamental aspect of UML-RT which is overlooked by the existing attempts to formalize its semantics. To illustrate the semantic importance of this issue we now present an example that highlights how thread allocation affects the semantics. This example also illustrates several of the UML-RT features that our proposed formal semantics addresses.

Example 1 Suppose that some system $A$ uses some subcomponent $B$ to perform a task, but $B$ may fail to answer requests timely. For such situations, $A$ includes an optional sub-component $C$ as a fall-back. At first, $A$ will attempt to make a request to $B$, and if $B$ responds, then it will continue to behave in some specified way. But if $B$ has not responded within a certain amount of time, $A$ will send the request to $C$, while still listening to a possible response from $B$. If a response from $C$ arrives, the behaviour of $A$ will continue in a different way than if the response came from $B$.

The model is shown in Figure 4. In this model we have a top-level capsule $A$ with a fixed sub-capsule $B$ and an optional sub-capsule $C$. $A$ is connected to $B$ via the $l_{1}$ connector, so ports $p_{1}$ (internal) of $A$ and $p_{3}$ of $B$ are wired. However ports $p_{2}$ of $A$ and $p_{4}$ of $C$ are unwired. Their behaviour is as follows: capsule $A$ registers $p_{2}$ as an SAP under some service name "s" and incarnates a capsule in $C$ in some logical thread $L_{1}$. Then it sets up a timer to trigger in 1.0 time units, and 


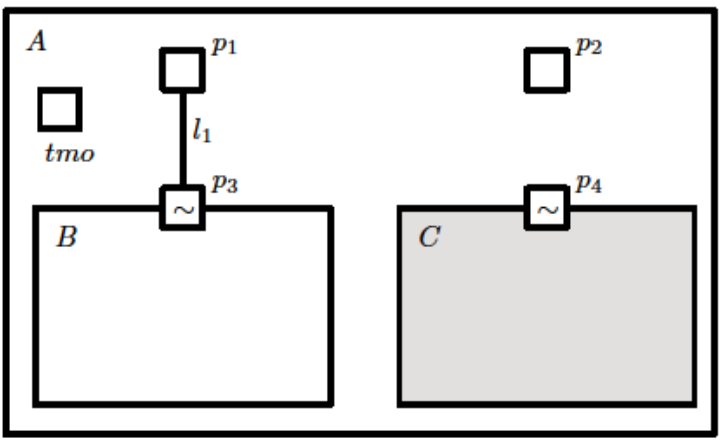

(a) Structure diagram

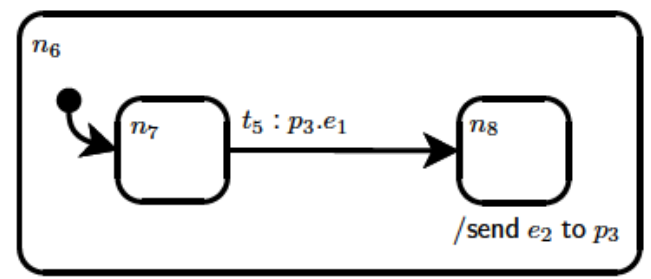

(c) State machine diagram for capsule $B$

Fig. 4 Model sensitive to thread assignment.

sends an event $e_{1}$ to $B$ via $p_{1}$. If it receives a response from $B$ on port $p_{1}$, it goes to state $n_{4}$. After the timeout event is received on port tmo, it sends event $e_{1}$ to $C$ via port $p_{2}$ and waits for a reply from either $B$ on $p_{1}$ or $C$ on $p_{2}$, leading to states $n_{4}$ or $n_{5}$ respectively. Capsule $B$ simply waits for input from $A$ on port $p_{3}$ and then responds on the same port with an event $e_{2}$. Capsule $C$ is very similar to $B$, except that in its initial state it registers its port $p_{4}$ as an SPP under the service name "s", thus connecting it to $A$ 's internal port $p_{2}$. Then it waits for input on $p_{4}$ and responds on the same port with an event $e_{2}$. All actions in Figure 4 are assumed to be entry actions.

The standard assumption in the literature is maximum concurrency: each capsule is executed by its own thread. In particular, the logical thread $L_{1}$ in which $C$ is incarnated is executed in a separate physical thread. Under such assumption, there are many possible behaviours. Each capsule will have its own event pool, and therefore transitions and actions can be interleaved in any order, so it is possible that $B$ performs transition $t_{5}$ before $C$ performs transition $t_{6}$, leading to $A$ ending up in state $n_{4}$, but it is also possible that $C$ performs transition $t_{6}$ before $B$ performs transition $t_{5}$, causing $A$ to end up in state $n_{5}$.

However, if $C$ is incarnated in the same physical thread as $B$ (the logical thread $L_{1}$ is associated to the same physical thread of $B$ ), then the behaviour becomes deterministic, as both $B$ and $C$ will share the same controller and therefore the same event pool. Since $A$ always sends a message to $B$ before sending a message

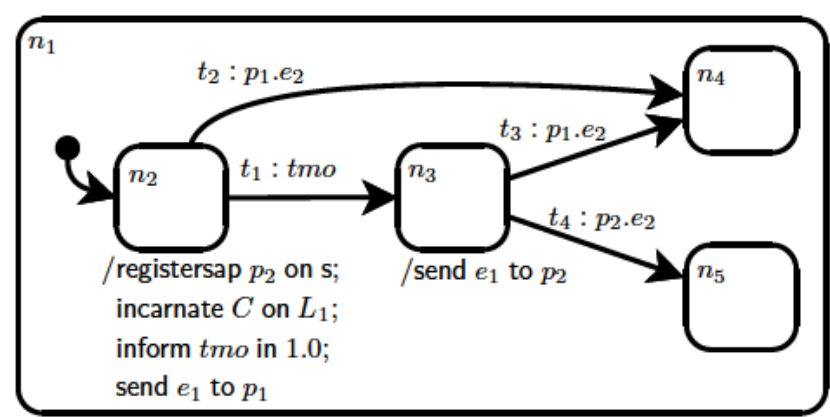

(b) State machine diagram for capsule $A$

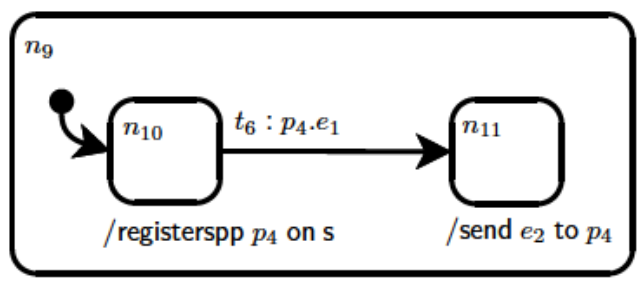

(d) State machine diagram for capsule $C$

to $C$, it is guaranteed that the message addressed to $B$ will be in the queue before the message addressed to $C$ (assuming the same priorities). Therefore, transition $t_{5}$ of $B$ is always triggered first, and the entry action of state $n_{8}$ of $B$ is executed as part of the same runto-completion step. Hence $B$ sends event $e_{2}$ back to $A$ before $C$ does, which implies that transition $t_{2}$ or $t_{3}$ of $A$ is triggered and $A$ always ends up in state $n_{4}$. This implies that under this thread assignment, no execution of the system will lead to $A$ being in state $n_{5}$, with $B$ always winning the race over $C$. In other words, we get only unfair executions.

It is essential to point out that when $B$ and $C$ are assigned to the same thread, the system's behaviour is deterministic regardless of the platform. This model can be easily extended to show that if $B$ and $C$ are associated to the same thread, then the system will deadlock, whereas if they are associated to different threads, they may not deadlock. For example, we can add another transition from $n_{8}$ in $B$ which listens to a message $e_{3}$ from $A$ but this message is sent from state $n_{5}$ in $A$. In such a model, if $B$ and $C$ are associated to the same thread, then $A$ will never end up in state $n_{5}$ and therefore $B$ will never get to receive $e_{3}$. Similarly we can modify the example to show that under certain thread assignments the system is unfair while under others it may be fair. For example, if we add a loop in the form of a transition from $n_{4}$ to $n_{2}$ in $A$, and similar loops with transitions from $n_{8}$ to $n_{7}$ in $B$ and from $n_{11}$ to $n_{10}$ in $C$ with trigger $e_{4}$ so that these systems could run forever, and we add an action to $n_{5}$ in $A$ that sends $e_{3}$ to $C$, 
then it becomes evident that under the assignment of $B$ and $C$ to the same thread, $C$ gets stuck in $n_{11}$ so it never gets a chance to continue executing. In other words, the behaviour becomes unfair.

It is not hard to make a simple model which, by using synchronous communication, exhibits deadlocks when the sender and receiver are on the same thread, but is deadlock-free when they are assigned to different threads.

By ignoring thread assignment, existing proposals of formal semantics for UML-RT fail to discriminate between a system that will deadlock from a system that may not deadlock and it will fail to discriminate from a fair system and an unfair system.

Thread allocation is important in practice, as it is related to optimization an deployment. The engineer may choose between different allocations depending on available resources or platform constraints. But, as this example shows, naively assuming that thread allocation is only a matter of optimization, or that it is only a platform dependent issue is misleading at best, and may result in incorrect runtime-behaviours or incorrect analysis results at worst. Therefore, a truly useful formal semantics of UML-RT must take it into account. The semantics proposed in this paper accounts for thread assignment and therefore can distinguish between certain systems with respect to some safety, liveness and fairness properties that other semantics for UML-RT fail to distinguish.

\section{State Machines}

Now we begin the presentation of our semantics. In this section we show how to map UML-RT state machines into kiltera. We first introduce a syntax to describe these state machines in Subsection 4.1, and then we describe how to map them into $\pi_{k l t}$ processes in Subsection 4.2 .

\subsection{A syntax for UML-RT state machines}

We use a mathematical notation for state machines, adapted from [60], which allows us to define the mapping compositionally.

In the sequel we will use the following sets:

$-\mathcal{N}_{\text {states }}$ : the set of all possible state names; we use $n, n_{1}, n_{2}, \ldots, m, \ldots$ for elements in $\mathcal{N}_{\text {states }}$;

$-\mathcal{N}_{\text {enp }}$ : the set of all possible entry point names; we use $a, a_{1}, a_{2}, \ldots$ for elements in $\mathcal{N}_{\text {enp }}$;

$-\mathcal{N}_{\text {exp }}$ : the set of all possible exit point names; we use $b, b_{1}, b_{2}, \ldots$ for elements in $\mathcal{N}_{\text {exp }}$;

$-\mathcal{N}_{c p} \stackrel{\text { def }}{=} \mathcal{N}_{\text {enp }} \cup \mathcal{N}_{\text {exp }}$ : the set of all connection point names; we use $c, c_{1}, c_{2}, \ldots$ for connection points.
$-\mathcal{N}_{\text {ports }}$ : the set of all possible port names; we use $p, p_{1}, p_{2}, \ldots$ for elements in $\mathcal{N}_{\text {ports }}$;

$-\mathcal{N}_{\text {evt }}$ : the set of all possible event names including the "non-event" $\perp$, used to mark transitions without a trigger; we use $e, e_{1}, e_{2}, \ldots$ for elements in $\mathcal{N}_{\text {evt }}$;

- Trig: the set of all possible triggers: it is defined as $\mathcal{N}_{\text {ports }} \times \mathcal{N}_{\text {evt }}$. We write $p . e$ for $(p, e) \in$ Trig.

- Vals: is a set of possible data values.

- Guards: the set of possible transition guards (which are boolean expressions over port names, capsule attributes, and event data). We write $g, g_{1}, g_{2}, \ldots$ for guards.

- Acts: the set of all possible actions including the "non-action" $\perp$, i.e., the action that does nothing; we use $f, f_{1}, f_{2}, \ldots$ for transition actions, en for entry actions and $e x$ for exit actions in Acts;

$-\mathbb{B} \stackrel{\text { def }}{=}\{$ false, true $\}$ the set of boolean values;

$-\mathbb{N}$ : the set of natural numbers

Furthermore, we make the following assumptions about these sets:

- Every state and connection point is labelled with a unique name. If this is not the case, a simple traversal of the state machine can give unique names, for example by providing fully qualified names or attaching a unique id.

- For every state name $n \in \mathcal{N}_{\text {states }}$, there is an entry point name $\operatorname{den}_{n} \in \mathcal{N}_{\text {enp }}$ and an exit point name $\operatorname{dex}_{n} \in \mathcal{N}_{\text {exp }}$. These denote the default entry and exit points of a state respectively, this is, when state $n$ is the target of a transition, but the transition is not connected to any named entry point, it is assumed to be connected to the default entry point $\operatorname{den}_{n}$. Analogously, when $n$ is the source of a transition, and the transition doesn't leave the state from a named exit point, it is assumed to begin at the default exit point $\operatorname{dex}_{n}$.

Before we define state machine terms, we define the encoding of transitions, which link connection points. We distinguish between three kinds of transition: incoming, outgoing and sibling. Incoming transitions are transitions from an entry point to some sub-state. Outgoing transitions are transitions from a sub-state to an exit point. Sibling transitions are transitions between sub-states.

Definition 2 (Transitions) Let Kinds $=\{$ in, out, sib $\}$ represent the set of transition kinds, (respectively in for incoming, out for outgoing, and sib for sibling). The set of all possible transitions is Trans $\stackrel{\text { def }}{=}$ Kinds $\times \mathbb{B} \times$ $\mathcal{N}_{c p} \times \mathcal{N}_{c p} \times$ Trig $\times$ Guards $\times$ Acts. Given a transition $t=\left(k, l, c_{1}, c_{2}, e, g, f\right) \in$ Trans we define the following 
functions: ${ }^{2}$

$$
\begin{aligned}
& \operatorname{kind}(t) \stackrel{\text { def }}{=} k \quad \text { The kind of transition } \\
& \text { first }(t) \stackrel{\text { def }}{=} l \quad \text { Whether } t \text { is the first in a chain } \\
& \operatorname{src}(t) \stackrel{\text { def }}{=} c_{1} \quad \text { The source of the transition } \\
& \operatorname{targ}(t) \stackrel{\text { def }}{=} c_{2} \quad \text { The target of the transition } \\
& \operatorname{trig}(t) \stackrel{\text { def }}{=} e \quad \text { The trigger event of the transition } \\
& \operatorname{guard}(t) \stackrel{\text { def }}{=} \mathrm{g} \quad \text { The guard of the transition } \\
& \operatorname{act}(t) \stackrel{\text { def }}{=} f \quad \text { The action of the transition }
\end{aligned}
$$

Now we can define state machine terms.

Definition 3 (State machine terms) The set SM of state machine terms is defined according to the following BNF:

$$
\begin{aligned}
& s::=[n, A, B, e n, e x] \quad \text { Basic-state } \\
& \text { | }[n, A, B, S, d, T, e n, e x] \text { Composite state }
\end{aligned}
$$

Here $n \in \mathcal{N}_{\text {states }}$ is the name of a state, $A \subseteq \mathcal{N}_{\text {enp }}$ and $B \subseteq \mathcal{N}_{\text {exp }}$ are the sets of entry and exit points where $A \cap B=\emptyset$ and $\operatorname{den}_{n} \in A$ and $\operatorname{dex}_{n} \in B$, en, ex $\in$ Acts are the entry and exit actions, $S$ is a sequence $\left\langle s_{1}, \ldots, s_{k}\right\rangle$ of sub-states with each $s_{i} \in \mathbf{S M}, d$ is the index, in the sequence, of the default sub-state $s_{d}$, and $T \subseteq$ Trans is a set of transitions subject to the conditions stated below.

We first define the following useful functions for a given basic state $s=[n, A, B, e x, e n]$ :

$$
\begin{aligned}
& \text { name }(s) \stackrel{\text { def }}{=} n \quad \text { The name of the state } s \\
& \text { entries }(s) \stackrel{\text { def }}{=} A \quad \text { The set of entry points of } s \\
& \operatorname{exits}(s) \stackrel{\text { def }}{=} B \quad \text { The set of exit points of } s \\
& \text { enact }(s) \stackrel{\text { def }}{=} \text { en The set of entry actions of } s \\
& \operatorname{exact}(s) \stackrel{\text { def }}{=} e x \quad \text { The set of exit actions of } s
\end{aligned}
$$

\footnotetext{
2 Note that since we assume unique names for all connection points, the source and target of a transition are well-defined.
}

For a composite state $s=[n, A, B, S, d, T, e n, e x]$ with $S=s_{1 . . k}$, we define

$$
\begin{aligned}
& \text { name }(s) \stackrel{\text { def }}{=} n \quad \text { The name of the state } s \\
& \text { entries }(s) \stackrel{\text { def }}{=} A \quad \text { The set of entry points } s \\
& \text { exits }(s) \stackrel{\text { def }}{=} B \quad \text { The set of exit points of } s \\
& \text { substates }(s) \stackrel{\text { def }}{=} S \quad \text { The set of substates of } s \\
& \operatorname{trans}(s) \stackrel{\text { def }}{=} T \quad \text { The set of transitions of } s \\
& \text { default }(s) \stackrel{\text { def }}{=} s_{d} \quad \text { The default (initial) substate of } s \\
& \text { enact }(s) \stackrel{\text { def }}{=} \text { en The set of entry actions of } s \\
& \operatorname{exact}(s) \stackrel{\text { def }}{=} e x \quad \text { The set of exit actions of } s
\end{aligned}
$$

and all transitions $t \in T$ must satisfy the following conditions:

1. If first $(t)=$ false then $\operatorname{trig}(t)=\perp$

2. $\operatorname{kind}(t)=$ sib if and only if there are sub-states $s_{i}$ and $s_{j}$ in $S$ such that $\operatorname{src}(t) \in \operatorname{exits}\left(s_{i}\right)$ and $\operatorname{targ}(t) \in$ entries $\left(s_{j}\right)$.

3. $\operatorname{kind}(t)=$ in if and only if there is a sub-state $s_{i}$ in $S$ such that $\operatorname{src}(t) \in A$ and $\operatorname{targ}(t) \in \operatorname{entries}\left(s_{i}\right)$.

4. $\operatorname{kind}(t)=$ out if and only if there is a sub-state $s_{i}$ in $S$ such that $\operatorname{src}(t) \in \operatorname{exits}\left(s_{i}\right)$ and $\operatorname{targ}(t) \in B$.

In the remainder we will omit the entry and exit actions when $e n=\perp$ and $e x=\perp$, and if we omit a transition's guard, it is assumed to be true. Also, in our examples, the transition's labels have the general form $t: p . e[g] / a$ where $t$ is the transition's name (only used for readability, but not part of the formal definition), p.e $\in$ Trig is the transition's trigger with port $p \in \mathcal{N}_{\text {ports }}$ and event $e \in \mathcal{N}_{\text {evt }}, g \in$ Guards is the transition's guard, and $a \in$ Acts is the transition's action. All of these items are optional.

Example 2 Consider the state machine shown in Figure 5. This is encoded in our syntax as follows:

$$
\begin{aligned}
& s_{1} \stackrel{\text { def }}{=}\left[n_{1},\left\{\operatorname{den}_{n_{1}}\right\},\left\{\operatorname{dex}_{n_{1}}\right\},\left\langle s_{2}, s_{5}\right\rangle, 1,\left\{t_{1}, t_{2}\right\}\right] \\
& s_{2} \stackrel{\text { def }}{=}\left[n_{2},\left\{\operatorname{den}_{n_{2}}, a_{1}, a_{2}\right\},\left\{\operatorname{dex}_{n_{2}}, b_{1}, b_{2}\right\}\right. \text {, } \\
& \left.\left\langle s_{3}, s_{4}\right\rangle, 1,\left\{t_{3}, t_{4}, t_{5}, t_{6}, t_{7}\right\}\right] \\
& s_{3} \stackrel{\text { def }}{=}\left[n_{3},\left\{\operatorname{den}_{n_{3}}\right\},\left\{\operatorname{dex}_{n_{3}}\right\}\right] \\
& s_{4} \stackrel{\text { def }}{=}\left[n_{4},\left\{\operatorname{den}_{n_{4}}, a_{3}\right\},\left\{\operatorname{dex}_{n_{4}}\right\}\right] \\
& s_{5} \stackrel{\text { def }}{=}\left[n_{5},\left\{\operatorname{den}_{n_{5}}\right\},\left\{\operatorname{dex}_{n_{5}}\right\}\right]
\end{aligned}
$$




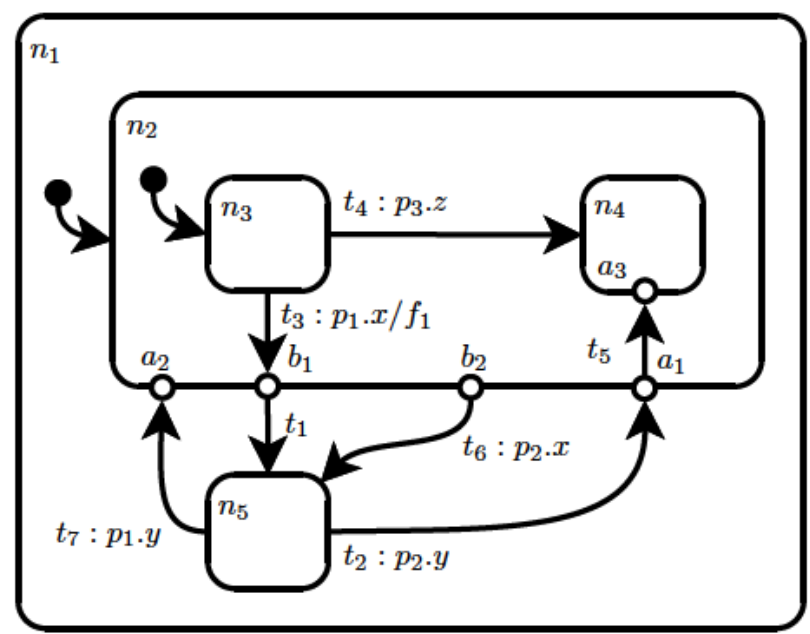

Fig. 5 A state machine with composite states.

with transitions

$t_{1} \stackrel{\text { def }}{=}\left(\right.$ sib, false, $\left.b_{1}, \operatorname{den}_{n_{5}}, \perp, \perp\right)$

$t_{2} \stackrel{\text { def }}{=}\left(\right.$ sib, true, $\left.\operatorname{dex}_{n_{5}}, a_{1}, p_{2} . y, \perp\right)$

$t_{3} \stackrel{\text { def }}{=}$ (out, true, $\operatorname{dex}_{n_{3}}, b_{1}, p_{1} . x, f_{1}$ )

$t_{4} \stackrel{\text { def }}{=}\left(\mathrm{sib}\right.$, true, $\left.\operatorname{dex}_{n_{3}}, \operatorname{den}_{n_{4}}, p_{3} . z, \perp\right)$

$t_{5} \stackrel{\text { def }}{=}$ (in, false, $a_{1}, a_{3}, \perp, \perp$ )

$t_{6} \stackrel{\text { def }}{=}$ (sib, true, $b_{2}$, den $_{n_{5}}, p_{2} . x, \perp$ )

$t_{7} \stackrel{\text { def }}{=}\left(\right.$ sib, true, $\left.\operatorname{dex}_{n_{5}}, a_{2}, p_{1} . y, \perp\right)$

\subsection{Translating state machine diagrams}

\subsubsection{An informal description}

\section{State hierarchy}

We begin by describing how the nesting order of a state machine is represented in $\pi_{k l t}$. The essence of the idea is to encode states as process definitions and we obtain the hierarchical structure via nested process definitions. Each state $n_{i}$ will be encoded as a process definition named $S n_{i}$. Recall that $\pi_{k l t}$ process definitions can be nested with the def $\{\ldots\}$ in $P$ construct. We make use of this feature, to place the definitions of sub-states inside the definition of a composite state. Nested definitions are only half of the story. The other half is instantiation: to enter a state we invoke its definition. If the state is composite, when we instantiate it, it will in turn, invoke (in parallel) the definitions of the sub-state it is entering. The scoping rules of $\pi_{k l t}$ guarantee encapsulation.

\section{Simple transitions}

Events are triples $\langle p, e, d\rangle$ where $p . e \in$ Trig is an event trigger, and $d \in$ Vals is some (optional) data associated with the event. The process for a state contains a subprocess called the Handler which receives these events on the state's inp port. The decision of which transition to take based on this event, is made by a process called Choice, which has a branch for each possible (sibling or outgoing) transition from the state. The result will (normally) be to execute the transition's action and then invoke the process $S n_{j}$ where $n_{j}$ is the target of the corresponding transition.

\section{Entry and exit points}

We represent entry points of a state via a parameter enp of the process definition for that state. When the state is entered, this parameter is used to invoke the sub-state connected to the corresponding entry point. The component of the state in charge of making this decision and choosing the sub-state to activate is called the Dispatcher.

Exit points are simpler to represent than entry points. For basic states we don't need to represent them at all. For composite states, we represent them if there is an outgoing transition from some sub-state to the exit point. The exit point acts as a pseudo-state, an intermediate point between the actual source of the transition chain and its actual target. Hence, in our representation we create a simple process that immediately jumps to the destination. We use the convention of naming the definition for an exit point $b$ as $B b$. Hence, if the target of a transition segment is an exit point $b_{j}$, the process will execute $B b_{j}$, which will itself continue with the next segment in the transition chain. In addition to jumping to the destination, the $B b_{j}$ process must also inform its containing state that it is exiting, so that the containing state becomes inactive (and more specifically, to stop the state's Handler).

\section{Group transitions}

A group transition is a transition whose source is (an exit point of) a composite state. When taking such a transition, the currently active sub-state of the composite state, becomes (recursively) inactive.

Traditionally, group transitions are usually interpreted by flattening the state machine and adding a corresponding transition to every sub-state of the group transition's source. We take a different approach to preserve modularity. First, we add, in every sub-state, a pair of events exit and exack, which are used, respectively, to tell the state to exit, and to acknowledge the exit from that state. Second, in the composite state that is the source of the group transition whenever one 
such event occurs, the Handler tells its currently active sub-state to exit and then waits for the sub-state to acknowledge the exit before jumping to the actual destination. Waiting for the sub-state to exit ensures that the sequence of exit actions will be executed in the correct order.

\section{Enabled-transition selection policy}

It is possible that two transitions are simultaneously enabled if their source is the currently active state and they share the same trigger event. In this case the transitions are said to be in conflict. If the source of one such transition is a sub-state of the source of the other transition, then the conflict is resolved by giving priority to the former, inner transition (SVP 1). In this section we implement such priority scheme. Note that this "priority" is different from the priority of events in the event pool. Such event priorities will be addressed later.

The main idea is as follows. For each composite state $n$, the Handler receives the incoming event and before it compares this event with the triggers of the transitions from $n$, it forwards the event "down" to its currently active sub-state $n^{\prime}$. If $n^{\prime}$ (or a sub-state) has a transition with this event as a trigger, then it handles the event and sends an "accepted" message back to n's Handler. On the other hand, if $n^{\prime}$ (or a sub-state) didn't have such a transition, then it sends a "rejected" message back to $n$ 's Handler. If $n$ 's Handler receives from $n^{\prime}$ an "accepted" message, it in turn sends an "accepted" message to its containing state. If it receives a "rejected" message, it compares the event with the triggers of $n$ 's transitions. If one trigger matches, an "accepted" message is sent to the containing state of $n$ and the transition is taken. Otherwise, a "rejected" message is sent.

In order to implement this, we add an acc and a rej port to inform the containing state of acceptance or rejection of events.

\section{History}

Whenever a composite state is entered for the first time, its initial sub-state is entered. If, however, the composite state was previously visited, and the composite state is entered through an entry point not explicitly connected to any sub-state, it enters the last visited sub-state, i.e., the sub-state which was active when the composite state exited. This behaviour is called history. The policy applies recursively for the sub-state, resulting in what is known as deep history. (SVP 2)

To implement history we define, for each state $n_{k}$ a history cell $h_{k}$, a process which stores $n_{k}$ 's last visited sub-state. In fact, whenever we take a transition inside a composite state $n_{k}$, we store the target state of the transition $n_{k}$ 's history cell, and hence, $h_{k}$ always contains $n_{k}$ 's currently active sub-state. Then, if we exit $n_{k}$ and reenter it later, the Dispatcher recalls the state stored in the history cell.

\section{Actions}

There are two main issues to be addressed in order to support actions: first, how are individual actions encoded in $\pi_{k l t}$ and second, when should they be executed?

To address the first question, we consider an existing set of actions Acts without specifying what are these actions exactly. Normally these actions would be given in some action language (SVP 3). However, the order of execution (the second issue) is independent of such action language, and therefore it is useful to keep this set abstract, and assume that we have a translation $\alpha$ : Acts $\rightarrow$ KLT which maps each action to the corresponding $\pi_{k l t}$ term. Later on we will provide a specific action language (Subsection 5.1.2) and a specific translation in the context of UML-RT capsules (Subsection 5.2.6).

Once we assume the action translation, we can focus on where to put the resulting translations. We have three kinds of action: entry actions, exit actions and transition actions. Entry actions must be executed whenever we enter a state. Similarly, exit actions must be executed whenever we exit a state. Transition actions are executed whenever the transition is taking place, after exiting the source state and before entering the target state. This means that the process $S n$ for a state $[n, \ldots, e n, e x]$ must begin by executing $\alpha(e n)$ and that $\alpha(e x)$ must be executed when leaving the state, in process $B b$ for each exit point $b$.

\subsubsection{Formal mapping}

\section{Actions}

As stated above, we need a translation for actions. The particular action language may vary, so we assume that an appropriate translation is provided.

Definition 4 (Action translation) An action translation is a map $\alpha:$ Acts $\rightarrow \mathcal{C} \rightarrow$ KLT from the set of possible actions Acts to the set of $\pi_{k l t}$ terms KLT, where $\mathcal{C}$ is some set of contextual information needed to do the translation. 


\section{History cells}

The history cell $h$ for a given state $m$ stores its last active sub-state $S n$, as well as a boolean flag ini, which is set to true if the state $m$ has been previously visited. History cells are instances of the following process:

Definition 5 (History Cells) History Cells are represented by the following process definition:

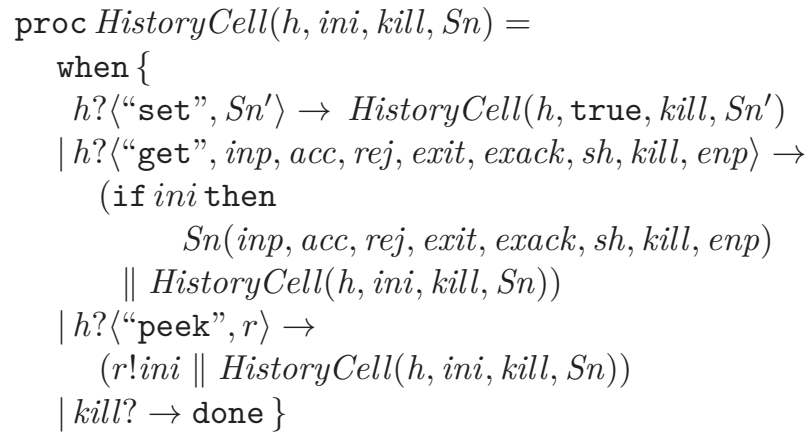

The way a history cell works is straight-forward. It accepts three kinds of messages: "set", to store a substate in the cell, "get" to execute the currently stored sub-state, and "peek" to determine whether the cell has been initialized. When a "set" message is received, it comes with the name $S n^{\prime}$ of the sub-state process to be stored in the cell. This is kept as the third parameter in the definition of HistoryCell. In this case, the ini flag is set to true, indicating that the cell has been initialized and the state has been visited at least once. When a "get" message arrives, if the state has been initialized, it executes the sub-state $S n$ currently stored, linking the ports and parameters passed along with the request. These parameters are explained below. Finally, when a "peek" message is received, it returns the value of the $i n i$ flag along a given channel $r$.

\section{The process definition for states}

Each state $n_{k}$ is translated into a process definition $S n_{k}$ which has the following ports and parameters:

- inp: this is the port where input events are received,

- acc: this port is used to signal that an input event has been accepted by the state,

- rej: this port is used to signal the rejection of an input event, i.e., , that the event cannot be handled by the state because no outgoing transition from this state is enabled by the event.

- exit: this port is used by the state's parent to request the state to exit,

- exack: this port is used to acknowledge an exit request, once the necessary (and possibly recursive) exit actions have been performed,
- sh: this port is used to signal that exiting this state also exits the enclosing state, and thus the Handler of the enclosing state must stop (hence $s h$ for "stop handler"),

- kill: this port is used to stop the state and all processes associated to it, including its Handler and sub-states.

- enp: is a parameter used only in composite states to pass the name of the entry point used to enter the state.

The difference between exit, kill and sh is as follows: exit is signaled when executing a group transition, so the composite state taking the transition asks its currently active sub-state to exit and waits for it to exit before executing the corresponding Exit action; kill is signaled when the entire state machine is being destroyed, when it's capsule is being destroyed so the composite state being killed asks its active sub-state to be killed as well, without executing exit actions or waiting for substates to finish; and $s h$ is signaled when a transition chain is being taken and going through an exit point so the handler of the composite state is to be stopped.

In the following, we assume that for each composite state $n_{k}$ there is a top-level channel $h_{k}$ for its history cell. We also assume a global event compl, used to indicate that we have reached a stable state, and thus signaling the end of a run-to-completion step. The following definition formalizes the translation of a basic state $s$ whose containing (parent) state is $s^{\prime}$, as

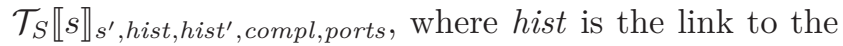
history cell for $s$, hist' is the history link for its parent $s^{\prime}$, compl is the completion event, and ports is the list of ports of the capsule containing the state machine.

Each transition is assumed to be annotated with a label $(p, e, g)$ where $p$ is a port name, $e$ is an event name and $g$ is a guard (a boolean expression). Incoming events are of the form $\langle p, e, d\rangle$ where $p$ is a port name, $e$ is an event name and $d$ is some data associated to the event $e$.

This translation assumes a translation for actions $\alpha:$ Acts $\rightarrow \mathcal{C}_{\text {ports }} \rightarrow \mathbf{K L T}$, with context set $\mathcal{C}_{\text {ports }}$ whose elements are pairs $\langle\langle p, e, d\rangle$, ports $\rangle$ of incoming events and lists of ports (so that the action can refer or use the event and/or the capsule's ports).

We now provide the definition of the translation $\mathcal{T}_{S} \llbracket s \rrbracket \ldots$ for basic states in Definition 6 and composite states in Definition 7.

Translation of basic states

Definition 6 (Translation of basic states) Given a basic state

$s=\left[n_{k}, A, B, e n, e x\right]$ 


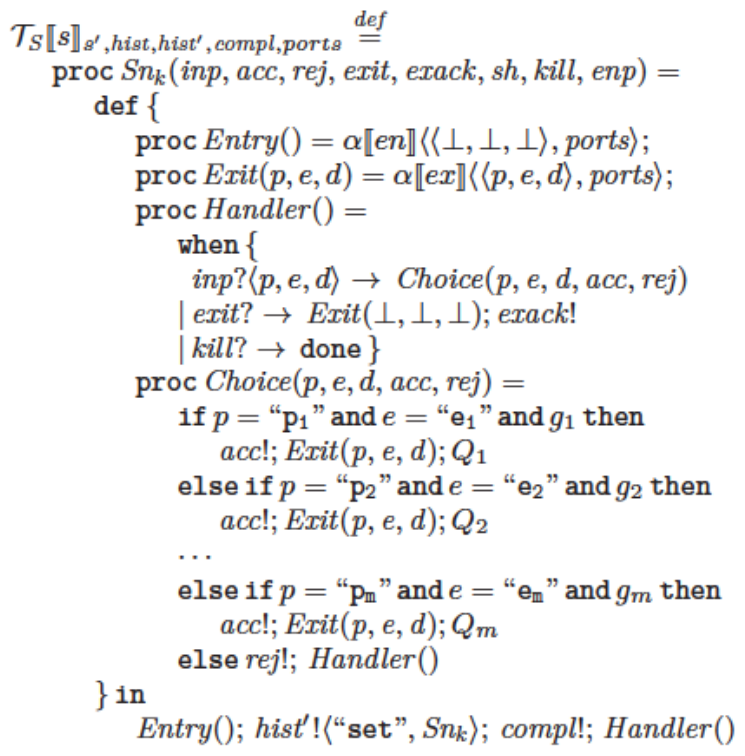

Fig. 6 Translation of basic states.

whose parent (enclosing) state is ${ }^{3}$

$s^{\prime}=\left[n_{k^{\prime}}^{\prime}, A^{\prime}, B^{\prime}, S^{\prime}, d^{\prime}, T^{\prime}, e n^{\prime}, e x^{\prime}\right]$

and given an action translation $\alpha:$ Acts $\rightarrow \mathcal{C}_{\text {ports }} \rightarrow$ KLT, the translation of $s$ is the $\pi_{k l t}$ term $\mathcal{T}_{S} \llbracket s \rrbracket \ldots$ shown in Figure 6, where for each outgoing transition $t_{i} \in$ $T^{\prime \prime}, \operatorname{trig}\left(t_{i}\right)=p_{i} \cdot e_{i}$, and $\operatorname{guard}\left(t_{i}\right)=g_{i}$ with $T^{\prime \prime} \stackrel{\text { def }}{=}$ $\left\{t \in T^{\prime} \mid \exists b \in B . b=\operatorname{src}(t)\right\}$ being the set of outgoing transitions, i.e., the transitions in the containing state $s^{\prime}$ whose source is an exit point of $s$, and where $Q_{i}$ is the process that executes the transition's action and goes to the target of the transition, defined as

$Q_{i} \stackrel{\text { def }}{=}\left\{\begin{array}{c}T_{i} ; S n_{j}(i n p, a c c, \text { rej, exit, exack, sh, kill, a }) \\ \quad \text { if } \operatorname{kind}\left(t_{i}\right)=\operatorname{sib}, a=\operatorname{targ}\left(t_{i}\right), \\ \quad \exists s_{j} \in S^{\prime} . a \in \operatorname{entries}\left(s_{j}\right), \text { and } n_{j}=\operatorname{name}\left(s_{j}\right) \\ T_{i} ; B b_{j}(s h) \\ \quad \text { if } \operatorname{kind}\left(t_{i}\right)=\text { out and } b_{j}=\operatorname{targ}\left(t_{i}\right) \in B^{\prime}\end{array}\right.$

and where each $T_{i}$ is the process which executes the action of transition $t_{i}$, namely $\alpha \llbracket \operatorname{act}\left(t_{i}\right) \rrbracket\langle(\perp, \perp, \perp)$, ports $\rangle$

\section{Explanation}

Figure 7 shows the control flow of the $\pi_{k l t}$ definition for basic states. In this definition, a basic state starts by executing its entry action Entry(), then it updates the history cell of its parent with the currently active state by doing $h i s t$ ! 〈"set", $\left.S n_{k}\right\rangle$. Hence the history cell for $s^{\prime}$

\footnotetext{
${ }^{3}$ If the state has no parent, i.e., , it is the top-most state on the state-machine, the role of the parent will be taken by a special process called Sink, described in Definition 8.
}

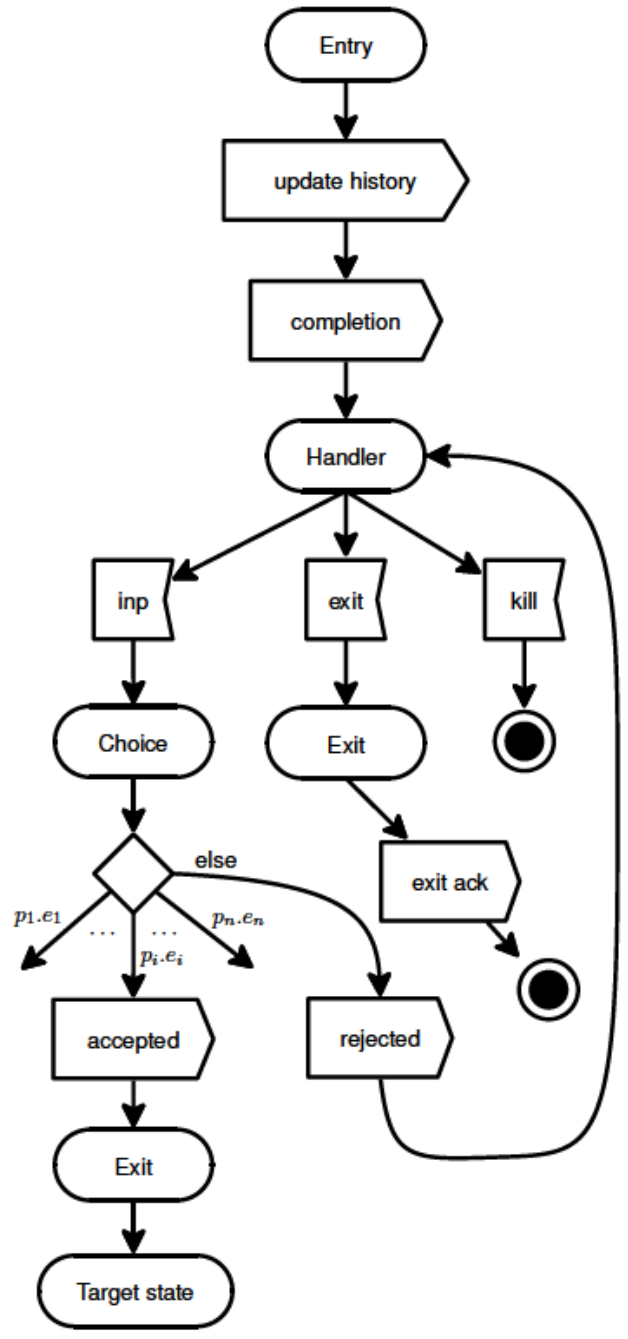

Fig. 7 Activity diagram for the control flow of basic states.

holds the currently active sub-state $s$. Note that hist' is the link to the parent's history cell., then we trigger the event compl, signaling that we have reached a stable state, thus ending a run-to-completion step, and then start the Handler process, which waits for input events.

The Handler waits for an input event on its inp port, or for an exit request. If an event arrives on the inp port, the decision of what to do with it is taken by the Choice process, which contains a conditional with a branch for each outgoing transition with trigger $p_{i} . e_{i}$ and guard $g_{i}$. If input comes from port $p_{i}$ with data event $e_{i}$ and its guard $g_{i}$ is true, the transition is taken: first, the acc event is triggered indicating that the event was accepted by the state; second, the exit action of the state (Exit) is executed, followed by the transition's action $\left(T_{i}\right)$; and finally control passes to the target of the transition, either some (sibling) state $S n_{j}$ (through entry point $a$ ) or some exit point $\left(b_{j}\right)$ of the containing state. 


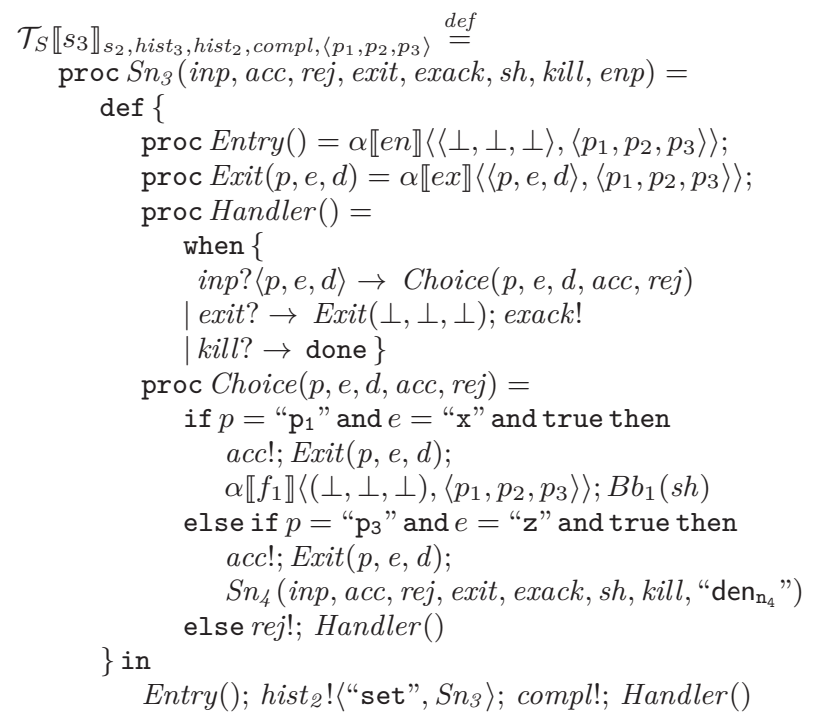

Fig. 8 Example: translation of state $n_{3}$ from Figure 5 .

Example 3 Consider the basic state $n_{3}$ from the state machine from Figure 5, inside state $s_{2}$ with outgoing transitions $t_{3}$ and $t_{4}$. Figure 8 shows the result of the translation $\mathcal{T}_{S} \llbracket s_{3} \rrbracket_{s_{2}}$, hist , $_{3}$, hist, , compl,ports where ports $=$ $\left\langle p_{1}, p_{2}, p_{3}\right\rangle$.

In this example one can see that the Choice process has three branches: one for transition $t_{3}$, one for transition $t_{4}$ and the default branch for the case when the incoming event does not match the trigger of these transitions and the event is rejected.

The first branch, corresponding to $t_{3}$, informs its containing state $\left(\mathrm{Sn}_{2}\right)$ that the event is accepted (acc!), executes the exit action, then executes the transition action $f_{1}$ and then executes the process corresponding to the exit point $b_{1}$ (process $B b_{1}$ ). The definition for $B b_{1}$ will be provided in the definition of the enclosing state $\mathrm{Sn}_{2}$.

The second branch is similar, but the transition does not have an action to execute, and the target state is $n_{4}$, thus it invokes the process $S n_{4}$ with entry point $\operatorname{den}_{n_{4}}$, the default entry point.

In the main body, the history cell for the parent, hist $_{2}$ is set to this state $S n_{3}$, after executing the entry action.

\section{Translation of composite states}

\section{Definition 7 (Translation of composite states)}

Given a composite state

$s=\left[n_{k}, A, B, S, d, T, e n, e x\right]$

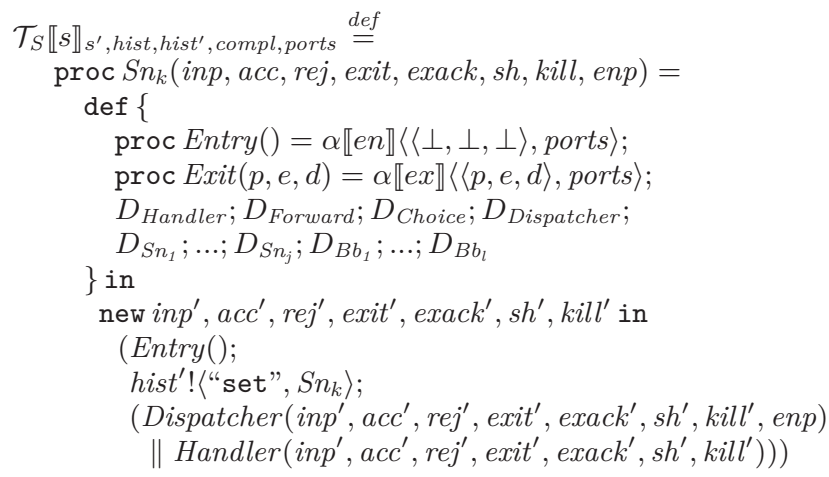

Fig. 9 Translation of composite states.

$$
\begin{aligned}
& \text { proc Dispatcher }\left(i n p^{\prime}, a c c^{\prime}, r e j^{\prime}, \text { exit }{ }^{\prime}, \text { exack }^{\prime}\right. \text {, } \\
& \left.s h^{\prime}, k i l l^{\prime}, e n p\right)= \\
& \text { if } \quad e n p=\text { " } \mathrm{a}_{1} \text { " then } Q_{1}^{\prime} \\
& \text { elseif enp }=\text { " } \mathrm{a}_{2} \text { " then } Q_{2}^{\prime} \\
& \text {... } \\
& \text { else if } e n p=\text { " } \mathrm{a}_{\mathrm{m}} \text { " then } Q_{m}^{\prime} \\
& \text { else hist! 〈"get", inp', acc', rej', exit', } \\
& \text { exack', sh', kill', enp }
\end{aligned}
$$

Fig. 10 Dispatcher: chooses a sub-state according to the entry point or history.

whose parent (enclosing) state is ${ }^{4}$

$s^{\prime}=\left[n_{k^{\prime}}^{\prime}, A^{\prime}, B^{\prime}, S^{\prime}, d^{\prime}, T^{\prime}, e n^{\prime}, e x^{\prime}\right]$

and given an action translation $\alpha: \operatorname{Acts} \rightarrow \mathcal{C}_{\text {ports }} \rightarrow$ KLT, the translation of $s$ is the $\pi_{k l t}$ term $\mathcal{T}_{S} \llbracket s \rrbracket \ldots$ shown in Figure 9 , where each $D_{S n_{i}}$ is the definition of substate $n_{i}$ :

$D_{S n_{i}} \stackrel{\text { def }}{=} \mathcal{T}_{S} \llbracket s_{i} \rrbracket_{s, h_{i}, h_{k}, \text { compl,ports }}$

with $h_{i}$ being the history cell for sub-state $n_{i}$ (state term $s_{i}$ ), $h_{k}$ being the history cell for $n_{k}$ (state term $s) ; D_{B b_{j}}$ is the definition of exit point $b_{j}$ given below; $D_{\text {Handler }}, D_{\text {Forward }}, D_{\text {Choice }}$ and $D_{\text {Dispatcher }}$ are the definitions of the Handler, Forward, Choice and Dispatcher given below:

- The Dispatcher process definition $D_{\text {Dispatcher }}$ is given in Figure 10, where as before, $Q_{i}^{\prime}$ is the target of the transition segment $i$, the process that executes the transition's action and goes to the target of the transition, defined as

$Q_{i}^{\prime} \stackrel{\text { def }}{=}\left\{\begin{aligned} T_{i} ; S n_{j}\left(i n p^{\prime}, a c c^{\prime}, r e j^{\prime}, \text { exit }^{\prime}, \text { exack }^{\prime}, \text { sh }^{\prime}, k i l l^{\prime}, a\right) \\ \quad \text { if } \operatorname{kind}\left(t_{i}\right)=\operatorname{in}, a=\operatorname{targ}\left(t_{i}\right), \\ \exists s_{j} \in S . a \in \operatorname{entries}\left(s_{j}\right), \text { and } n_{j}=\operatorname{name}\left(s_{j}\right) \\ T_{i} ; B b_{j}\left(s h^{\prime}\right) \\ \quad \text { if } \operatorname{kind}\left(t_{i}\right)=\text { out and } b_{j}=\operatorname{targ}\left(t_{i}\right) \in B\end{aligned}\right.$

\footnotetext{
${ }^{4}$ If the state has no parent, i.e.,, it is the top-most state on the state-machine, the role of the parent will be taken by a special process called Sink, described in Definition 8.
} 


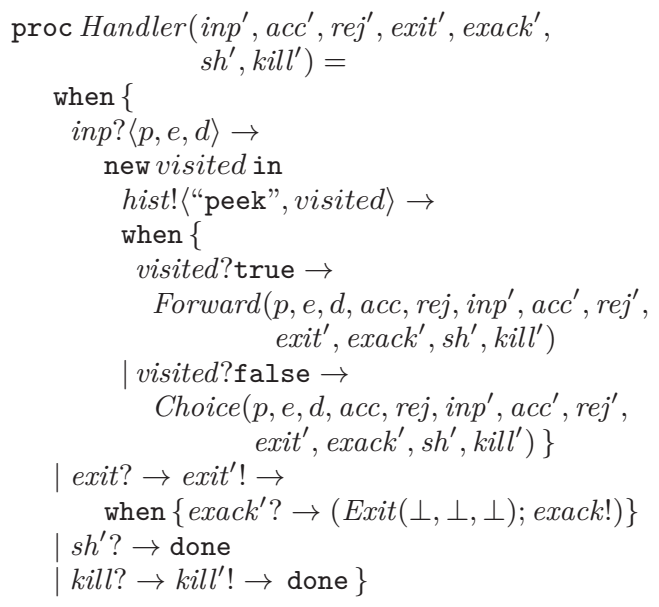

Fig. 11 Composite state handler.

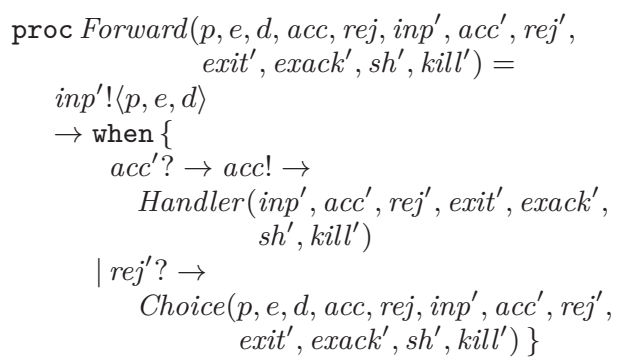

Fig. 12 Composite state event-forwarder.

and where each $T_{i}$ is the process that executes the action of transition $t_{i}, \alpha \llbracket \operatorname{act}\left(t_{i}\right) \rrbracket\langle(\perp, \perp, \perp)$, ports $\rangle$.

- $D_{B b_{j}}$ is a process definition for exit point $b_{j} \in B$, given by

$$
D_{B b_{j}} \stackrel{\text { def }}{=} \operatorname{proc} B b_{j}(s h)=s h ! \| Q_{j}
$$

where $s h$ is the parent's stop-handler signal, and $Q_{j}$ is the target of the exit point, defined as follows:

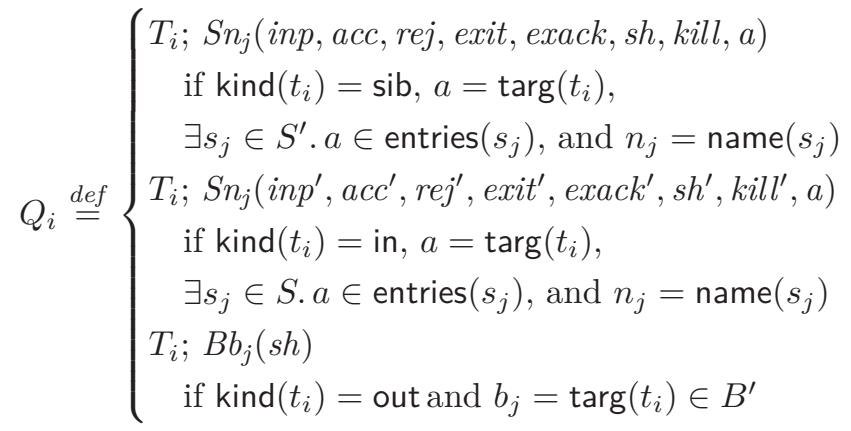

- $D_{\text {Handler }}$ is the process definition shown in Figure 11.

- $D_{\text {Forward }}$ is the process definition shown in Figure 12.

- Finally, $D_{\text {Choice }}$ is the process definition shown in Figure 13, where $Q_{i}$ is the process that executes the

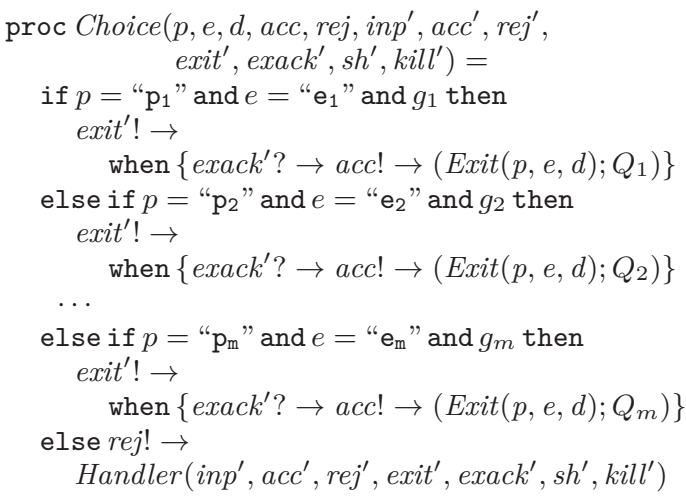

Fig. 13 Composite state choice taker.

transition's action and goes to the target of the transition, defined (in the same way as for $B b_{j}$ above) as

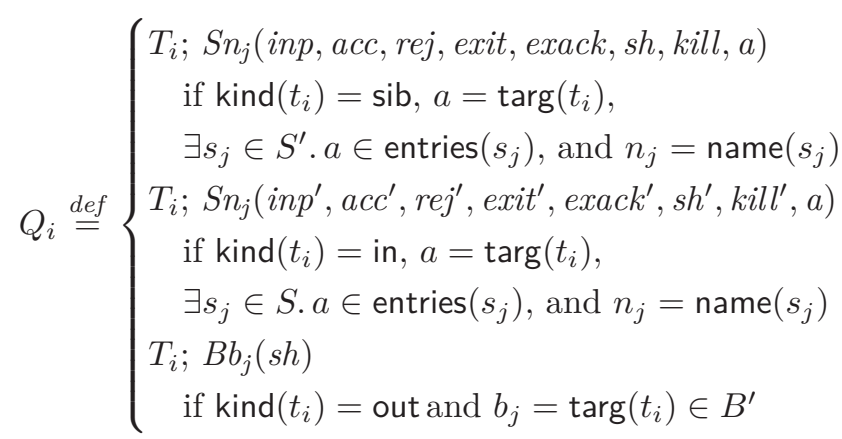

\section{Explanation}

Figure 14 shows the control flow of the $\pi_{k l t}$ definition for composite states. As with basic states, the definition of a composite state contains definitions for entry and exit actions (Entry and Exit) respectively, an event handler (Handler) and a process to make the choice of what to do with the event (Choice). In addition to these, it also contains:

- a dispatcher to either follow an incoming transition into some sub-state or recall history (Dispatcher),

- a definition $D_{S n_{i}}$ for each sub-state $n_{i}$,

- a definition $D_{B b_{j}}$ for each exit point $b_{j}$

- and a forwarder process which forwards incoming events down to the currently active sub-state, in order to implement the "deepest first" enabled-transition selection policy.

In the main body, the state begins by executing its entry action $(\operatorname{Entry}())$, then it updates the history cell of its parent with the currently active state by doing hist'! "set", $\left.S n_{k}\right\rangle$, and then starts the Dispatcher and the event Handler. Both Dispatcher and Handler are invoked with primed channels $i n p^{\prime}, a c c^{\prime}, r e j^{\prime}$, exit', exack', sh', and $k i l l^{\prime}$, which are used to interact with 


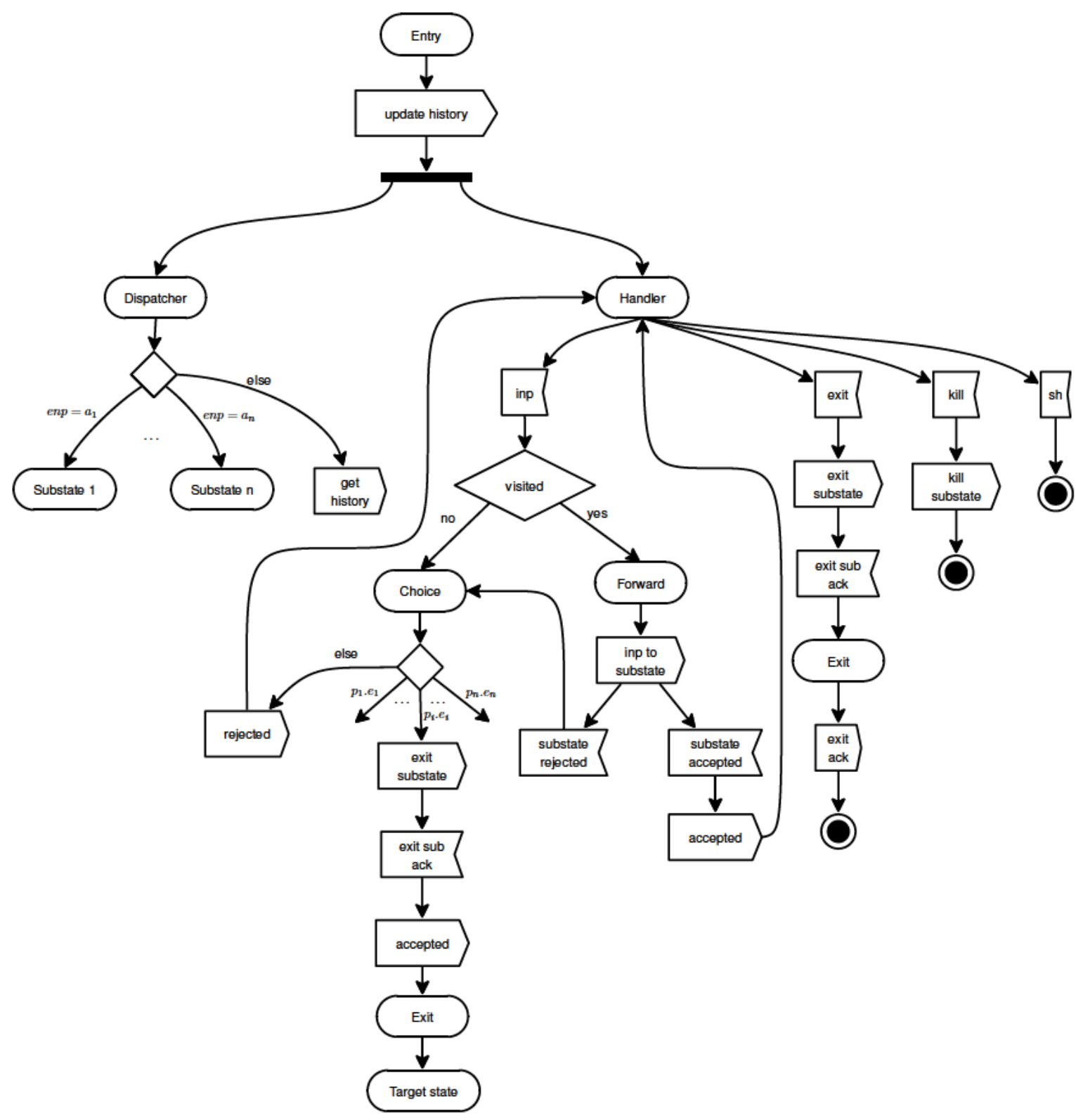

Fig. 14 Activity diagram for the control flow of composite states.

the currently active sub-state. For example, inp is the channel where the composite state $n_{k}$ receives its input events and $i n p^{\prime}$ is the channel where $n_{k}$ forwards input events to its currently active sub-state (which may change).

The Dispatcher chooses which sub-state to activate (invoke) based on the value of the enp parameter which must contain the name of an entry point. If it is a valid entry point name, it represents an incoming transition $i$, thus $Q_{i}^{\prime}$ is performed, which executes the transition's action and invokes the process corresponding to the target of the transition, passing as parameters the current primed channels $i n p^{\prime}, a c c^{\prime}, r e j^{\prime}, e x i t^{\prime}, e x a c k^{\prime}, s h^{\prime}$, and kill' so that the newly activated sub-state can interact with the Handler ${ }^{5}$. If it is not a named entry point, the history cell for the state is recalled, also passing as parameters the current primed channels $i n p^{\prime}, a c c^{\prime}, r e j^{\prime}$, exit', exack', sh', and $k i l l^{\prime}$.

${ }^{5}$ Note that the processes $Q_{i}$ and $Q_{i}^{\prime}$ are very similar but differ in the parameters passed to the target state: $Q_{i}^{\prime}$ deals with incoming transitions, so the primed channels $i n p^{\prime}, a c c^{\prime}, r e j^{\prime}$, exit', exack', sh', and kill' are passed to the target sub-state so that it may interact with state $n_{k}$ 's Handler. On the other hand, $Q_{i}$ deals with both incoming and sibling transitions, and therefore, in the sibling transition case, it passes on the nonprimed channels inp, acc, rej, exit, exack, sh, and kill so that the target state becomes the currently active sub-state of its parent and thus can communicate with the parent's Handler. 
The event Handler is somewhat more complex than that for basic states. It accepts three kinds of events: inp (input events), exit, and sh (stop Handler). On an input event, the Handler inquires its history cell if it has been previously visited. (SVP 4)

If the state has been previously visited, (the history cell has been initialized with some sub-state) it proceeds to the normal case handled by the Forward process, which forwards the event down to the currently active sub-state (via $i n p^{\prime}$ ) and waits to see if it was accepted $\left(a c c^{\prime}\right)$ or rejected $\left(r e j^{\prime}\right)$. If accepted by the sub-state, the acceptance is forwarded "up" to the parent $(a c c)$, and we wait for the next event. If rejected by the substate, we attempt to handle the event at this level in the Choice process. (SVP 5)

If the state has not been previously visited, no substate has been activated, which is the case if there is no initial transition to some sub-state. In this case, the composite state remains "on the border", and thus, events are not forwarded to any sub-state and are handled by the Choice process.

The exit event (exit) might be received from the parent state, in which case the request is forwarded to the currently active sub-state via exit' and when acknowledgement from the sub-state arrives ( exack $^{\prime}$ ), the exit action is executed (Exit) and an acknowledgement is forwarded to the parent (exack).

Finally, the stop Handler signal (sh) may be received when leaving the state via an exit point $b_{j}$. In this case it is not necessary to ask the currently active sub-state to stop, because it was precisely that substate who has executed the outgoing transition and has performed its own exit sequence. Note that if a transition chain is executed, going through several exit points, each exit point triggers an sh event to stop the Handler of the composite state containing the exit point, thus, all Handlers in the chain are stopped as expected. The kill event is much like the stop-Handler event, but is used to stop the states in a "top-down" fashion: whenever the Handler receives a kill request, it kills its currently active sub-state by triggering $k_{i l l}^{\prime}$ and then stops. This will be used later, in the mapping for capsules, to kill a state machine when we destroy its capsule.

Example 4 Let us revisit the example from Figure 5. Consider the composite state $n_{2}$ in that machine. The definitions for the Handler and Forward processes are fixed, only the definitions for Dispatcher, Choice and the sub-states, as well as the exit points are specific.

First we have the Dispatcher. There are three possible ways to enter the state: through entry points $a_{1}$ or $a_{2}$ or through the default entry point, in which case his- tory will be recalled, or if the state has not been visited before, the initial state.

$$
\begin{aligned}
& \text { proc Dispatcher }\left(i n p^{\prime}, a c c^{\prime}, r e j^{\prime},\right. \text { exit', exack', } \\
& \left.s h^{\prime}, k i l l^{\prime}, \text { enp }\right)= \\
& \text { if enp }=\text { " } \mathrm{a}_{1} \text { " then }
\end{aligned}
$$

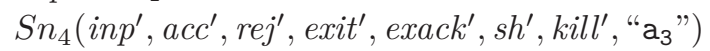

$$
\begin{aligned}
& \text { else hist! 〈"get", inp', acc', rej', exit', exack', } \\
& \left.s h^{\prime}, k i l l^{\prime}, \text { enp }\right\rangle
\end{aligned}
$$

In the case where we enter the state through $a_{1}$, we have to take transition $t_{5}$, which has no action, and then go to state $n_{4}$, invoking $S n_{4}$, and enter $n_{4}$ through entry point $a_{3}$. In the case where we enter through $a_{2}$, we have to recall history because there is no incoming transition connecting $a_{2}$ to any sub-state. Similarly, for the default entry point we recall history. Note that when we recall history, if it is the first time, the resulting state will be the initial state.

Second we have the choice process. There is only one (group) transition coming out of $n_{2}$, namely $t_{6}$. Thus the choice process is as follows:

$$
\begin{aligned}
& \text { proc Choice (p,e,d,acc, rej, inp' }{ }^{\prime}, a c c^{\prime}, r e j^{\prime} \text {, } \\
& \text { exit } \left.{ }^{\prime}, \text { exack }{ }^{\prime}, \text { sh }^{\prime}, k i l l^{\prime}\right)= \\
& \text { if } p=\text { " } \mathrm{p}_{2} \text { " and } e=\text { " } \mathrm{x} \text { " and true then } \\
& \text { exit' } ! \rightarrow \text { when }\left\{\text { exack }^{\prime} ? \rightarrow \text { acc! } \rightarrow(\operatorname{Exit}(p, e, d) \text {; }\right. \\
& \left.\left.S n_{5}\left(\text { inp, acc, rej, exit, exack, sh, kill, " } \operatorname{den}_{\mathrm{n}_{5}}{ }^{\prime}\right)\right)\right\} \\
& \text { else rej! } \rightarrow \text { Handler }\left(i n p^{\prime}, a c c^{\prime}, r e j^{\prime},\right. \\
& \text { exit', exack', sh', kill') }
\end{aligned}
$$

Note that the parameters passed to $S n_{5}$ are the unprimed ports, as this is a sibling transition, so we pass the channels used by $n_{2}$ and $n_{5}$ to communicate with their common parent $\left(n_{1}\right)$, so that $S n_{1}$ can now interact with the new currently active sub-state $S n_{5}$.

Definitions for the exit points are also generated. The only one actually invoked by a sub-state is for $b_{1}$ :

$$
\begin{aligned}
& \operatorname{proc} B b_{1}(s h)= \\
& \quad s h ! \| S n_{5}\left(\text { inp, acc, rej, exit, exack, sh, kill, "den } \mathrm{n}_{5}\right. \text { ") }
\end{aligned}
$$

This is executed when taking transition $t_{1}$ (after $t_{3}$ ). It sends a signal sh to the Handler process of $S n_{2}$ to stop it (the parameter $s h$ is given when exiting $n_{3}$; see the Choice process in Example 3, Figure 8). Then the process jumps to the target state $S n_{5}$.

The rest of the definition of $S n_{2}$ consists of the definitions of $S n_{3}$ (Example 3, Figure 8) and $S n_{4}$.

The process definition of a full state machine

Having defined processes for basic and composite states, we are now in a position to define the process for a whole 
state machine, which acts essentially as a wrapper, providing history cells, a top level state, links to the capsule containing the state machine, a Sink process to catch the acceptance and acknowledgment events from the top-level state, and a process to handle kill requests, so when a kill event comes in, the states and history cells are stopped.

A full state machine is represented as a process StateMachine with the following ports:

- inp: where input events of the form $\langle p, e, d\rangle$ are received (where $p$ is the port, $e$ is the event, and $d$ is some data value),

- compl: where the state machine signals the end of a run-to-completion step, when a stable state has been reached,

- kill: where requests to end the processes of the state machine are received,

$-e_{1}^{\prime}, \ldots, e_{n_{E}}^{\prime}, i_{1}^{\prime}, \ldots, i_{n_{I}}^{\prime}$ : the containing capsule's (output) ports, where the actions of the state machine can send events.

The formal specification is as follows:

Definition 8 (Translation of a full state machine) Let $s \in \mathbf{S M}$ be a state machine term. Its translation as a full state machine is $\mathcal{T}_{S M} \llbracket s \rrbracket$, given by

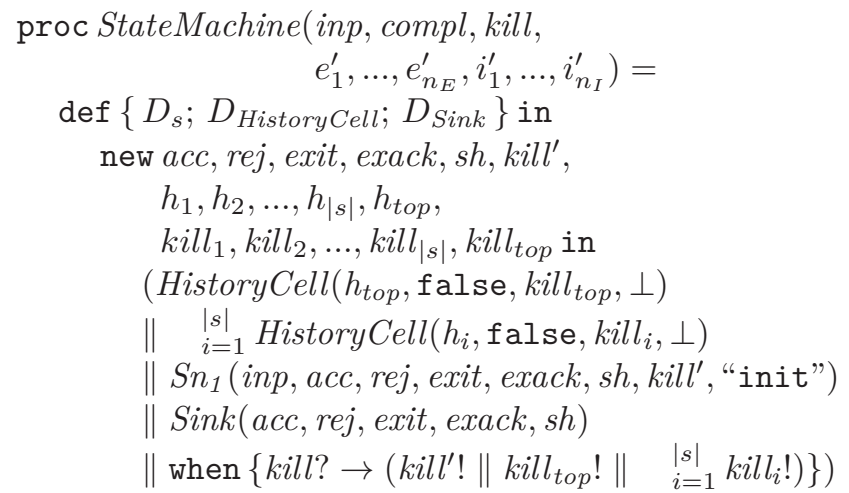

where

$-\operatorname{name}(s)=n_{1}$

- $|s|$ is the number of states in $s$, including all substates,

- $h_{i}$ is a link to the history cell for state $n_{i}$

- D History Cell is the definition of HistoryCell from Definition 5,

- $D_{s}$ is the translation of $s$ according to Definition 6 or Definition 7:

$D_{s} \stackrel{\text { def }}{=} \mathcal{T}_{S} \llbracket s \rrbracket_{\text {top }, h_{1}, h_{\text {top }}, \text { compl,ports }}$

with ports $\stackrel{\text { def }}{=}\left\langle e_{1}^{\prime}, \ldots, e_{n_{E}}^{\prime}, i_{1}^{\prime}, \ldots, i_{n_{I}}^{\prime}\right\rangle$, and where top is a dummy container state term defined as top $\stackrel{\text { def }}{=}$ $\left[n_{\text {top }}, \emptyset, \emptyset,\langle s\rangle, 1, \emptyset\right]$.
- and $D_{\text {Sink }}$ is the following definition:

$$
\begin{aligned}
& \operatorname{proc} \operatorname{Sink}(a c c, r e j, \text { exit, exack, sh })= \\
& \text { when }\{ \\
& a c c ? \rightarrow \operatorname{Sink}(a c c, r e j, \text { exit, exack, sh }) \\
& \mid r e j ? \rightarrow \operatorname{Sink}(\text { acc, rej, exit, exack, sh) } \\
& \mid \text { exack? } \rightarrow \operatorname{Sink}(a c c, \text { rej, exit, exack, sh) } \\
& \mid s h ? \rightarrow \operatorname{Sink}(\text { acc, rej, exit, exack, sh })\}
\end{aligned}
$$

\section{Capsules}

We now show how capsule diagrams are encoded as kiltera processes. In this section we begin by defining a syntax for UML-RT capsule diagrams and models (Subsection 5.1) including an action language. Then we define the translation for this syntax (Subsection 5.2).

The translation describes how to:

- associate capsules to threads (Subsection 5.2.1)

- represent (thread) controllers (Subsection 5.2.2)

- represent capsules themselves (Subsection 5.2.3)

- represent ports and services (Subsection 5.2.4)

- represent optional and plug-in parts (Subsection 5.2.5)

- represent actions (Subsection 5.2.6)

- represent the timer (Subsection 5.2.7)

- put all these together (Subsection 5.2.8)

\subsection{A syntax for UML-RT capsule diagrams}

We use a mathematical notation for capsule diagrams, which allows us to define the mapping compositionally.

In the sequel we will use the following sets:

$-\mathcal{N}_{\text {cap }}$ : the set of all possible capsule names; we use $m, m_{1}, m_{2}, \ldots$ for elements in $\mathcal{N}_{\text {cap }}$;

$-\mathcal{N}_{\text {parts }}$ : the set of all possible part names; we use $b, b_{1}, b_{2}, \ldots$ for elements in $\mathcal{N}_{\text {parts }}$

$-\mathcal{N}_{\text {ports }}$ : the set of all possible port names; we use $p, p_{1}, p_{2}, \ldots$ for elements in $\mathcal{N}_{\text {ports }}$;

- $\mathcal{N}_{\text {conn }}$ : the set of all possible connector names; we use $l, l_{1}, l_{2}, \ldots$ for elements in $\mathcal{N}_{\text {conn }}$;

$-\mathcal{N}_{s m}$ : the set of all possible state machine names; we use $n, n_{1}, n_{2}, \ldots$ for elements in $\mathcal{N}_{s m}$;

- $\mathcal{N}_{\text {lthr }}$ : the set of all possible logical threads; we use $L, L_{1}, L_{2}, \ldots$ for elements in $\mathcal{N}_{\text {lthr }}$;

- SM: the set of all state machine terms (defined in Subsection 4.1); $\mathbf{S M}_{\perp} \stackrel{\text { def }}{=} \mathbf{S M} \cup\{\perp\}$ is the set of state machine terms extended with the "none" value $\perp$, representing the absence of a state machine.

- Vals: the set of possible values (data transmitted with events between capsules).

Furthermore, we make the following assumptions about these sets: 
- Every capsule is labelled with a unique name. If this is not the case, a simple traversal of the capsule diagram can give unique names, for example by providing fully qualified names or attaching a unique id.

- Within a capsule, port names and connector names are unique.

Before we define capsule diagram terms, we define the the syntax for port references and connectors. We distinguish between qualified and unqualified port references. The former are used to refer to a port of a subcapsule within the capsule of interest, while the latter is used to refer to a port of the capsule itself.

Definition 9 (Port references and connectors) We define the set Portref of port references according to the following BNF, with $F \in$ Portref:

\section{$F::=p \quad$ Unqualified port reference \\ | m.p Qualified port reference}

where $p \in \mathcal{N}_{\text {ports }}$ and $m \in \mathcal{N}_{\text {cap }} \cup \mathcal{N}_{\text {parts }}$.

We also define the set Conn of possible connectors according to the following BNF, with $k \in$ Conn:

$k::=l: F \rightarrow F$ Relay or internal connector

where $l \in \mathcal{N}_{\text {conn }}$ is the name of the connector, and $F \in$ Portref is a port reference. For a connector $k$ we define the following useful functions:

name $\left(l: F_{1} \rightarrow F_{2}\right) \stackrel{\text { def }}{=} l \quad$ The name of the connector $\operatorname{src}\left(l: F_{1} \rightarrow F_{2}\right) \stackrel{\text { def }}{=} F_{1} \quad$ The source of the connector $\operatorname{targ}\left(l: F_{1} \rightarrow F_{2}\right) \stackrel{\text { def }}{=} F_{2} \quad$ The target of the connector

\subsubsection{Capsules}

Now we can define capsule diagram terms. A capsule is fully defined by providing:

- A name,

- Its ports (end, relay and internal)

- An optional state machine

- A set of (sub-capsule) parts

- A set of connectors between ports

The following definition formalizes this by providing syntax for capsule terms.

Definition 10 (Capsule diagram terms) The set CAP of capsule diagram terms is defined according to the BNF shown in Figure 15, where:

- $m \in \mathcal{N}_{\text {cap }}$ is the name of a capsule,

- $G$ is a set of pairs $p_{i}: w_{i} g_{i}$ where $p_{i} \in \mathcal{N}_{\text {ports }}$ is a port name, $w_{i} \in\{\mathrm{w}, \mathrm{u}\}$ and $g_{i} \in\{\mathrm{end}$, int, rel $\}$ is its type,
$c::=[m, G, s, P, K, A]$
Capsule
$G::=\left\{p_{1}: w_{1} g_{1}, \ldots, p_{n}: w_{n} g_{n}\right\} \quad$ Ports (or gates)
$w::=\mathrm{w} \quad$ Wired port
$g::=$ end
Unwired port
rel
External end port
Internal port
External relay port
$P::=\left\{b_{1}: o_{1} m_{1}, \ldots, b_{n}: o_{n} m_{n}\right\} \quad$ Sub-capsule parts
$\begin{aligned} o::= & \text { fix } \\ & \text { opt } \\ & \text { plug }\end{aligned}$
Fixed role
Optional role
Plugin role
$K::=\left\{k_{1}, \ldots, k_{n^{\prime}}\right\}$
Local connectors
$A::=\left\{a_{1}, \ldots, a_{n^{\prime \prime}}\right\}$
Attribute names

Fig. 15 Syntax of UML-RT capsule diagrams.

- $s \in \mathbf{S M} \cup\{\perp\}$ is a state machine term (or $\perp$ if the capsule has no state machine), (see Definition 3)

- $P$ is the set of sub-capsule parts of $n$, more precisely a set of triples $b_{i}: o_{i} m_{i}$ where $b_{i} \in \mathcal{N}_{\text {parts }}$ is a part name, $o_{i}$ is the part's role and $m_{i} \in \mathcal{N}_{\text {cap }}$ is a capsule name,

- $K \subseteq$ Conn is a set of connectors subject to the conditions stated below,

- and $A$ is a set of attribute names.

We first define the following useful functions to extract the elements of a given capsule $c=[m, G, s, P, K, A]$ :

$$
\begin{array}{ll}
\text { name }(c) \stackrel{\text { def }}{=} m & \text { The name of the capsule } \\
\operatorname{ports}(c) \stackrel{\text { def }}{=} G & \text { The set of ports of the capsule } \\
\operatorname{capsm}(c) \stackrel{\text { def }}{=} s & \text { The capsule's state machine } \\
\operatorname{parts}(c) \stackrel{\text { def }}{=} P & \text { The set of sub-capsules } \\
\operatorname{conn}(c) \stackrel{\text { def }}{=} K & \text { The set of port connectors } \\
\operatorname{attrs}(c) \stackrel{\text { def }}{=} A & \text { The set of attribute names }
\end{array}
$$

Furthermore, we also have some functions to extract particular types of ports:

$$
\begin{gathered}
\operatorname{endports}(c) \stackrel{\text { def }}{=}\{p \mid p: w \text { end } \in G\} \\
\operatorname{intports}(c) \stackrel{\text { def }}{=}\{p \mid p: w \text { int } \in G\} \\
\operatorname{relports}(c) \stackrel{\text { def }}{=}\{p \mid p: w \text { rel } \in G\} \\
\text { extports }(c) \stackrel{\text { def }}{=} \text { endports }(c) \cup \text { relports }(c) \\
\text { wiredports }(c) \stackrel{\text { def }}{=}\{p \mid p: \mathrm{w} g \in G\} \\
\text { unwiredports }(c) \stackrel{\text { def }}{=}\{p \mid p: \mathrm{u} g \in G\}
\end{gathered}
$$


We generalize these functions over sets in the natural way. For example, if $C=\left\{c_{1}, c_{2}, \ldots, c_{n}\right\}$ is a set of capsules, then name $(C) \stackrel{\text { def }}{=}\left\{\right.$ name $\left.\left(c_{i}\right) \mid c_{i} \in C\right\}$ is the set of names of all capsules in the set. Similarly for the rest of these functions.

We assume that fix is the default role, so $b: m$ is the same as $b:$ fix $m$. We use the notation $m . b$ to refer to the part $b$ of capsule $m$, i.e., , $m . b=m^{\prime}$ iff $b: o m^{\prime} \in$ parts $(c)$. We write pcallroleb $=o$ if $b: o m \in \operatorname{parts}(c)$.

$$
\begin{aligned}
& \text { fixedcaps }(c) \stackrel{\text { def }}{=}\{b: \text { fix } m \in P\} \\
& \operatorname{optcaps}(c) \stackrel{\text { def }}{=}\{b: \text { opt } m \in P\} \\
& \text { plugincaps }(c) \stackrel{\text { def }}{=}\{b: \text { plug } m \in P\}
\end{aligned}
$$

We also write $l: F_{1} \leftrightarrow F_{2} \in K$ to mean that either $l: F_{1} \rightarrow F_{2} \in K$ or $l: F_{2} \rightarrow F_{1} \in K$. All connectors in $K$ must satisfy the following conditions:

1. if $l: p \leftrightarrow F \in K$ where $p$ is an unqualified port reference, then $p \in \operatorname{relports}(c) \cup \operatorname{intports}(c)$

2. if $l: m . p \leftrightarrow F \in K$ where $m . p$ is a qualified port reference, then there is a sub-capsule $c_{i} \in C$ such that name $\left(c_{i}\right)=m$ and $p \in \operatorname{extports}\left(c_{i}\right)$

3. every port $p \in \operatorname{relports}(c) \cup \operatorname{intports}(c)$ is linked to at most one connector $k \in K$ (possibly none).

4. no port $p \in$ endports $(c)$ is linked to any connector inside $c$.

5. for all connectors $l: m_{1} \cdot p_{1} \leftrightarrow m_{2} \cdot p_{2},\left\{p_{1}, p_{2}\right\} \subseteq$ wiredports $(c)$

6. for every port $p \in$ wiredports $(c)$ there is a $l: m . p \leftrightarrow$ $F \in K$

7. for every port $p \in$ unwiredports $(c)$ there is no $l$ : $m . p \leftrightarrow F \in K$.

If any component of a capsule diagram is not specified, we write it as - . This is useful for describing partially specified models, or abstracted models.

Note that this definition does not include any reference to protocols. This is by design. Our goal is to provide a behavioural semantics of UML-RT models, but protocols play a static, syntactic role in UML-RT. Protocols can be understood as port types. As future work we will formalize such a type system, but we leave it out of our present formalization as it would distract us from the behavioural aspects.

Definition 11 (Capsule models) The set UMLRT capsule models is defined by the following BNF:

$U::=\left[c_{0}, c_{1}, \ldots, c_{n}\right]$ UML-RT model

where $c_{0}$ is designated the model's top-capsule, and such that for each capsule $c_{i}$, for each part $b: o m_{j} \in$ $\operatorname{parts}\left(c_{i}\right)$, there is a capsule $c_{j}$ such that $m_{j}=\operatorname{name}\left(c_{j}\right)$, this is, all sub-capsules must be defined in the model.
Example 5 Consider the capsule diagram from Figure 1. Suppose that in that diagram, capsule $B$ is fixed, capsule $C$ is optional and capsule $D$ is a plug-in part. Furthermore, suppose that $s_{1}$ is the term representing the state machine of the capsule according to the syntax from Definition 3. Then the model is represented by

$U \stackrel{\text { def }}{=}\left[c_{1}, c_{2}, c_{3}, c_{4}\right]$

where each $c_{i}$ is a term representing the capsules as follows:

$$
\begin{gathered}
c_{1} \stackrel{\text { def }}{=}\left[A,\left\{p_{1}: \text { w rel, } p_{2}: \text { u end, } p_{3}: \text { u rel, } p_{4}: \text { wint }\right\}\right. \\
s_{1}, \\
\left\{b_{1}: \text { fix } B, b_{2}: \text { opt } C, b_{3}: \text { plug } D\right\}, \\
\left\{l_{1}: p_{1} \rightarrow B \cdot p_{5}, l_{2}: p_{4} \rightarrow C \cdot p_{8},\right. \\
l_{3}: p_{3} \rightarrow D \cdot p_{11}, l_{4}: B \cdot p_{6} \rightarrow C \cdot p_{9}, \\
\left.\left.l_{5}: B \cdot p_{7} \rightarrow D \cdot p_{10}\right\}, \emptyset\right] \\
c_{2} \stackrel{\text { def }}{=}\left[B,\left\{p_{5}: \mathrm{w}-, p_{6}: \mathrm{w}-, p_{7}: \mathrm{w}-\right\},-,-,-, \emptyset\right] \\
c_{3} \stackrel{\text { def }}{=}\left[C,\left\{p_{8}: \mathrm{w}-, p_{9}: \mathrm{w}-, p_{13}: \mathrm{u}-\right\},-,-,-, \emptyset\right] \\
c_{4} \stackrel{\operatorname{def}}{=}\left[D,\left\{p_{10}: \mathrm{w}-, p_{11}: \mathrm{w}-, p_{12}: \mathrm{u}-\right\},-,-,-\emptyset\right]
\end{gathered}
$$

We note the following. First, in the set of connectors, we can use either the capsule name or the name of the part $\left(b_{i}\right)$ in qualified port references, as per Definition 9 , so for example $l_{1}: p_{1} \rightarrow B . p_{5}$ could have been written $l_{1}: p_{1} \rightarrow b_{1} . p_{5}$. Also, the direction of the arrow is not relevant, as messages can flow in either direction, so we could have written $l_{1}: B . p_{5} \rightarrow p_{1}$. We also allow a bidirectional arrow as well: $l_{1}: p_{1} \leftrightarrow B . p_{5}$, however the designer may intend a specific direction for information flow, so the arrow can be used as a suggestion. This does not have an impact on the translation. Finally, a particular wiring of the capsules ports has been assumed.

Also note that in capsules $c_{2}, c_{3}$ and $c_{4}$ we have left the kinds of ports, state machines, parts and connectors unspecified. We could interpret this as a partially specified model, or an abstracted model. However, to obtain the actual semantics with our translation, the model must be fully specified.

\subsubsection{An action language}

In UML-RT actions are used in state machines, but most significant actions perform operations related to capsules, such as sending messages, or creating new capsules. For this reason, we introduce a syntax for actions in this section.

The syntax presented here includes only a subset of all possible operations in UML-RT. Nevertheless, these seem to form a core subset of actions. 


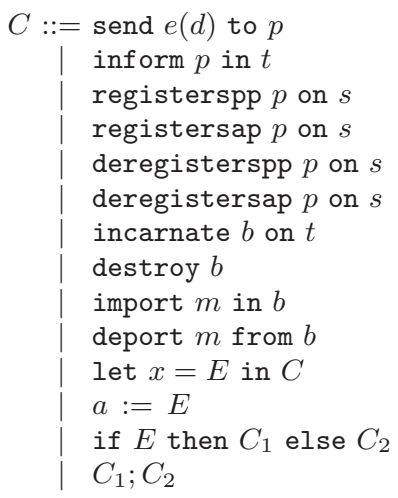

Fig. 16 Syntax for the action language.

Definition 12 (Actions) The set Acts of all possible actions from Subsection 4.1 is defined according to the BNF shown in Figure 16, (SVP 3) where $C$ ranges over Acts, and where $p \in \mathcal{N}_{\text {ports }}, e \in \mathcal{N}_{\text {evt }}, d \in$ Vals, $b \in \mathcal{N}_{\text {parts }}$, and $t \in \mathcal{N}_{\text {lthr }}$. Expressions $E$ can include attribute access.

Informally, these actions do the following:

- send $e(d)$ to $p$ sends event $e$ with data $d$ through port $p$.

- inform $p$ in $t$ sets up a timeout event on port $p$ after $t$ seconds.

- registerspp $p$ on $s$ registers the unwired port $p$ as an SPP with unique service name $s$.

- registersap $p$ on $s$ registers the unwired port $p$ as an SAP with unique service name $s$.

- deregisterspp $p$ on $s$ deregisters the unwired port $p$ as an SPP with unique service name $s$.

- deregistersap $p$ on $s$ deregisters the unwired port $p$ as an SAP with unique service name $s$.

- incarnate $b$ on $t$ incarnates optional capsule part $b$ on logical thread $t$.

- destroy $b$ destroys optional capsule part $b$.

- import $m$ in $b$ imports capsule instance $m$ in plugin capsule role $b$.

- deport $m$ from $b$ removes capsule instance $m$ from plug-in capsule role $b$.

- let $x=E$ in $C$ declares a local variable $x$ initialized to the value of $E$ with scope $C$, and executes $C$.

$-a:=E$ assigns the value of expression $E$ to the capsule's instance attribute $a$.

- if $E$ then $C_{1}$ else $C_{2}$ executes $C_{1}$ if the value of $E$ is true, otherwise, executes $C_{2}$.

- $C_{1} ; C_{2}$ executes $C_{1}$ and then $C_{2}$.

\subsection{Translating capsule diagrams}

Mapping UML-RT models to $\pi_{k l t}$ involves the following:

- Mapping state machine diagrams to process definitions

- Mapping capsule diagrams to process definitions

- Representing UML-RT "controllers", which guide the execution of the system

- Representing the association of capsules to threads

Each of these issues is largely independent of the others, and thus the combined map has a modular structure. We describe each of these in the following subsections.

We begin by defining the association of capsules to threads in Subsection 5.2.1, then describe how to represent controllers in Subsection 5.2.2 followed by the encoding of capsules in Subsection 5.2.3. In Subsection 5.2.4 we detail the behaviour of ports and services. In Subsection 5.2.5 we deal with optional and plug-in parts. In Subsection 5.2.6 we translate the action language into $\pi_{k l t}$ terms. In Subsection 5.2.7 we define the timing mechanism. Finally, in Subsection 5.2.8 we define the full translation, integrating all of the above.

\subsubsection{Mapping capsules to threads}

In order to support some deployment requirements, in UML-RT it is possible to associate each capsule to a logical thread. Each logical thread can in turn be assigned to a physical thread. Each physical thread corresponds to exactly one controller, and each controller corresponds to exactly one physical thread. Hence, in addition to the UML-RT model, we must take into account:

- the map from capsules to logical threads, and

- the map from logical threads to physical threads

Since the assignment to physical threads determines the controller of a capsule, this assignment is semantically meaningful, as capsules associated to different controllers will be able to execute simultaneously and use separate event pools. On the other hand, multiple logical threads on the same physical thread behave just as one logical thread. Therefore what we are interested in is the composition of these two maps.

Let us assume that $\mathcal{N}_{\text {lthr }}$ denotes the set of possible logical thread names, and $\mathcal{N}_{\text {pthr }}$ denotes the set of physical thread names.

Definition 13 (Capsule-to-thread assignment) Let $N_{C} \subseteq \mathcal{N}_{\text {cap }}$ be a set of capsule names, $N_{L} \subseteq \mathcal{N}_{\text {lthr }}$ be a set of logical thread names, and $N_{P} \subseteq \mathcal{N}_{\text {pthr }}$ a set of physical thread names. A capsule-to-logical-thread 
assignment over $N_{C}$ and $N_{L}$ is a function $\theta_{L}: N_{C} \rightarrow$ $N_{L}$, i.e., a map from capsule names to logical-thread names. A logical-to-physical-thread assignment over $N_{L}$ and $N_{P}$ is a function $\theta_{P}: N_{L} \rightarrow N_{P}$, mapping logical thread names to physical threads. The capsule-to-thread assignment is the composition of these two maps: $\theta \stackrel{\text { def }}{=}$ $\theta_{P} \circ \theta_{L}: N_{C} \rightarrow N_{P}$. For convenience we also define CAP2TH as the set of all possible capsule-to-thread assignments.

This assignment is used in the translation by creating a new instance of a controller for each physical thread, and linking a capsule $m$ to the controller for the thread $\theta(m)$ (Subsection 5.2.8).

\subsubsection{Controllers}

Each capsule is associated with a controller. Controllers are objects which guide the execution of a capsule or set of capsules. Controllers are responsible for implementing the run-to-completion semantics. A controller contains an event pool, and thus, all capsules associated to it share the same event pool. Hence at any point in time, amongst all capsules associated to a controller, there will be only one capsule, or more precisely one state machine active, i.e., executing an event.

During execution, capsules send each other messages or events. An event is sent to a specific port in the target capsule, and may have additional data associated with it which is transmitted as part of the message.

In our mapping, capsule ports and connectors are represented as kiltera events or channels, so sending an event $e$ with data $d$ to a port $p$ will be represented as triggering the event $p$ with the pair $\langle e, d\rangle$ as parameter. On reception, if the port is a relay port, the message will go directly to the final receiver. If the port is an end port, the capsule must have a state machine. In this case, the receiving capsule forwards this information, the tuple $\langle p, e, d\rangle$ to its controller, to be queued so that it is processed when the controller decides. The capsule must also forward to the controller, the state machine's input port $s m i$, and the event smc where the machine will signal it has completed the execution of an event. The $s m c$ port is called ctrl in the definition of states (see Definition 6, Definition 7 and Definition 8). Thus, capsules send each other messages of the form $\langle e, d\rangle$, controllers queue messages of the form $\langle s m i, s m c, p, e, d\rangle$ and state machines expect messages of the form $\langle p, e, d\rangle$.

Definition 14 (Controller events and event pools) An inter-capsule message is a pair $\langle e, d\rangle$ where

$-e$ is the name of a UML-RT event $-d$ is a reference to some data object, carried by the event

A state machine input message is a triple of the form $\langle p, e, d\rangle$ where

$-p$ is the name of the target port in the receiving capsule,

$-e$ is the name of a UML-RT event,

$-d$ is a reference to some data object, carried by the event

A controller message is a tuple of the form:

$\langle s m i, s m c, p, e, d\rangle$

where:

- smi is the input port of the state machine that must deal with the event,

- $s m c$ is the state machine's event which signals completion,

$-p$ is the name of the target port in the receiving capsule,

$-e$ is is the name of a UML-RT event,

$-d$ is a reference to some data object, carried by the event

Controllers consist of two components: an event pool, and a Dispatcher.

An event pool process is a $\pi_{k l t}$ process which has the following interface:

proc EventPool(put, get)

where put is a port where events are received by the queue, get is a port to get and remove the first item in the queue. The event pool process in a controller expects, on port put a message of the form of controller messages described above. We do not provide a specific implementation of such process in order to leave open the particular queuing policy desired. (SVP 6)

A Dispatcher is a process that takes the first event available in the queue and forwards it to the appropriate capsule, more specifically to the target capsule's state machine. Since the event pool holds tuples of the form $\langle s m i, s m c, p, e, d\rangle$ which come with the channel $s m i$ to the target state machine, all the Dispatcher has to do is to forward the tuple $\langle p, e, d\rangle$ to that channel and wait for the event $s m c$ which signals that the state machine has finished processing the event. Once this $s m c$ event is received, the controller can process the next event in the queue. Since a new event is taken from the queue and dispatched only when the completion event has been received, the controller guarantees the run-to-completion semantics. 
Definition 15 (Controllers) A controller is an instance of the following process:

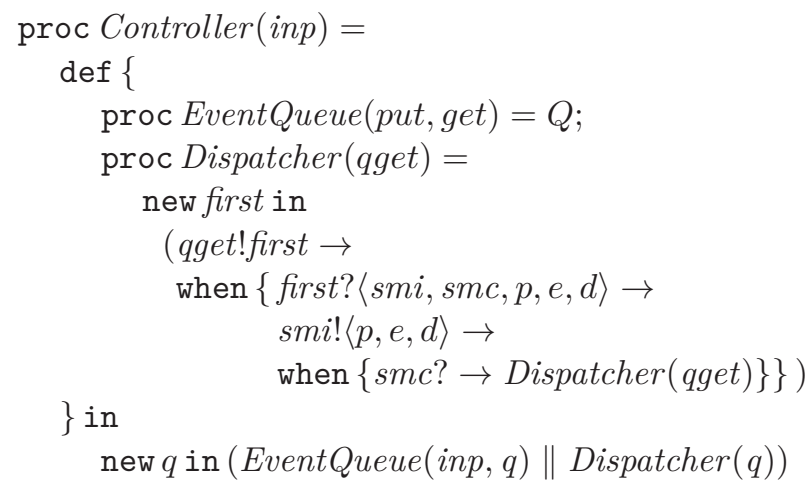

where $Q$ is the implementation of the event pool.

\subsubsection{Translating capsules}

Each capsule is represented as a single process definition which, when instantiated, contains:

- An instance of the capsule's state machine (called StateMachine)

- An instance of each sub-capsule (called $\mathrm{Cm}_{i}$ for each fixed capsule named $m_{i}$, Opt for each optional part and Plugin for each plug-in part.)

- An instance, for each port, of a port-handling process (called WiredEIPort and UnwiredEIPort depending on its type), and

- An instance of a process CapsuleHandler which handles operations on the capsule itself, such as those performed by actions in the action language,

Every UML-RT connector is represented by a pair of channels: one for sending messages in each direction. For each port/channel $p$ where input is expected, there is a port/channel $p^{\prime}$ which is used for output.

The interface of the capsule's process definitions contains a pair of ports for each end-port $\left(e_{p}, e_{p}^{\prime}\right)$ and relay-port $\left(r_{p}, r_{p}^{\prime}\right)$ of the capsule ${ }^{6}$, a ctrl port to link the capsule to its controller, and a hook channel where the capsule may receive certain instructions and queries. The hook channel is unique for each capsule, and thus can be thought of as the capsule's address or identifier.

Internally the definition includes the process definition corresponding to the state machine (this is called $D_{\text {StateMachine }}$ below), and a definition of the capsule handler $\left(D_{\text {CapsuleHandler }}\right)$. Additionally, there is a pair of local events/channels for each internal-port $\left(i_{p}, i_{p}^{\prime}\right)$

\footnotetext{
${ }^{6}$ In kiltera, channels are bidirectional, allowing both input and output on the same port. Nevertheless, we represent each UML-RT port (resp. connector) by a pair of kiltera ports (resp. channels) to differentiate between input and output on a port.
}

and and for each port connector $\left(l_{p}, l_{p}^{\prime}\right)$. There is also a $h o o k_{i}$ channel for each sub-capsule instance (fixed, optional or plug-in), a port handle $h_{p}$ linking each port $p$ to its port handler process, and a local variable $a_{i}$ for each attribute. Furthermore, there are local events/channels $s m i, s m c$, and $s m k$ representing, respectively, the statemachine's input and completion, this is, smi is where the state machine receives events, smc is where the state-machine signals that an event has been fully processed, smk used to kill the state machine when destroying the capsule.

The translation of a capsule $c$ is parametrized by an assignment $\theta \in \mathbf{C A P 2 T H}$ of capsule names to controllers, or more precisely to the input channel of the capsule's controller. We also assume a global event name sink, used as a receptor for unconnected ports.

We will use the following conventions for naming ports and channels:

- End ports will be written as $e, e^{\prime}, e_{1}, e_{1}^{\prime}, \ldots$

- Relay ports will be written as $r, r^{\prime}, r_{1}, r_{1}^{\prime}, \ldots$

- Internal ports will be written as $i, i^{\prime}, i_{1}, i_{1}^{\prime}, \ldots$

- Local connectors will be written as $l, l^{\prime}, l_{1}, l_{1}^{\prime}, \ldots$

- Capsule attributes will be written as $a, a_{1}, \ldots$

Definition 16 (Capsules to processes) Given some UML-RT model $U=\left[c_{0}, c_{1}, \ldots, c_{n}\right]$ and a capsule $c=$ $[m, G, s, P, K, A]$ in $U$, with endports $(c)=\left\{e_{1}, \ldots, e_{n_{E}}\right\}$, relports $(c)=\left\{r_{1}, \ldots, r_{n_{R}}\right\}, \operatorname{intports}(c)=\left\{i_{1}, \ldots, i_{n_{I}}\right\}$, parts $P=\left\{b_{1}: o_{1} m_{1}, \ldots, b_{n_{P}}: o_{n_{P}} m_{n_{P}}\right\}$, with each $m_{i} \stackrel{\text { def }}{=}$ name $\left(c_{i}\right)$ for some capsule $c_{i} \in U$, connectors $K=\left\{k_{1}, \ldots, k_{n_{K}}\right\}$, and attributes $A=\left\{a_{1}, \ldots, a_{n}\right\}$, we define $c$ 's translation into $\pi_{k l t}$ by the function $\mathcal{T}_{C} \llbracket \cdot \rrbracket$ : $\mathbf{C A P} \rightarrow \mathbf{C A P 2 T H} \rightarrow \mathbf{K L T}$, as shown in Figure 17, where:

$-L \stackrel{\text { def }}{=}\left\{\right.$ name $\left.\left(k_{i}\right) \mid k_{i} \in K\right\}=\left\{l_{1}, \ldots, l_{n_{K}}\right\}$

- D StateMachine is the translation of the capsule's state machine, if $s \neq \perp$, more precisely $D_{\text {StateMachine is }}$ $\mathcal{T}_{S M} \llbracket s \rrbracket$ according to Definition 8 (The $e_{p}^{\prime}$ and $i_{p}^{\prime}$ ports of the state machine are used by the state machine only to send events to sub-capsules $\left(i_{p}^{\prime}\right)$ or to other capsules $\left(e_{p}^{\prime}\right)$. All inputs to the state machine, including those from internal ports are received through the inp port of the state machine, as, according to the run-to-completion semantics, a state machine must handle one and only one input event at a time.)

- D CapsuleHandler is the definition of CapsuleHandler given in Definition 17,

- each $C m_{j}$ is the name of the process definition for the (sub)capsule named $m_{j}$, subject to the requirements described below, 


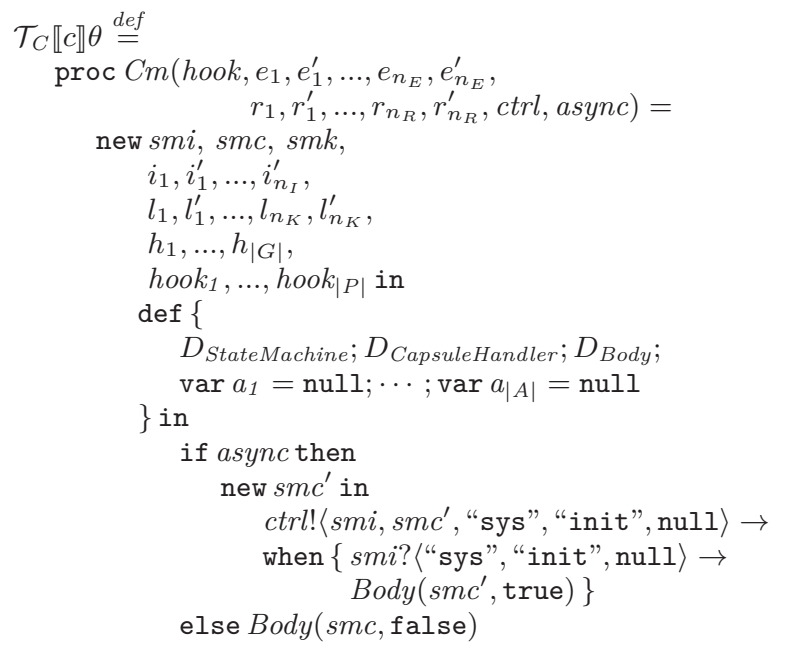

where $D_{\text {Body }}$ is

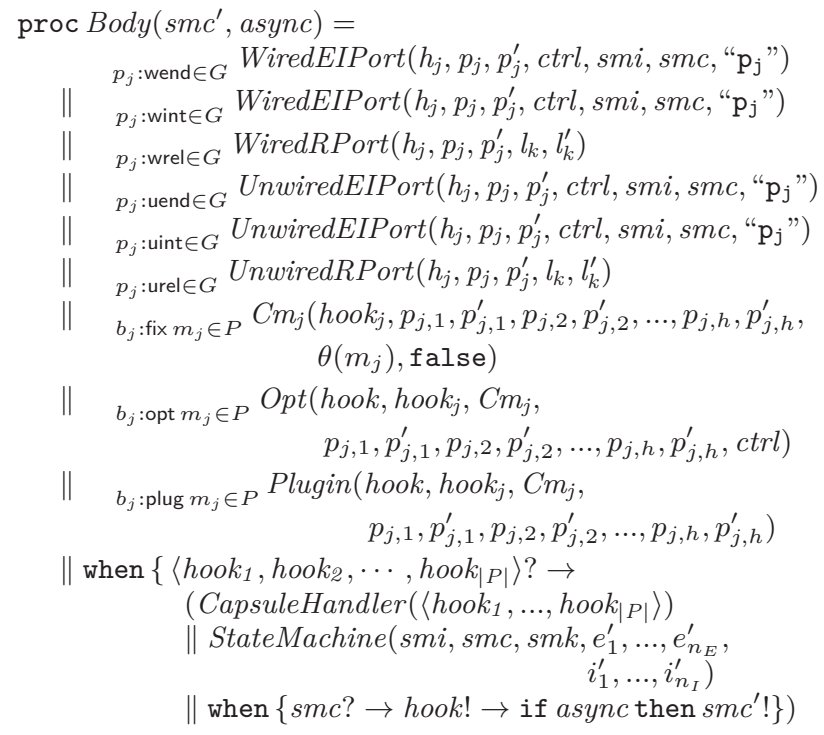

Fig. 17 Translation of capsules.

- the port handling process definitions WiredEIPort, WiredRPort, UnwiredEIPort and UnwiredRPort are given in Definition 18,

- the process definitions Opt and Plugin are given in Definition 20 and Definition 21 (Subsection 5.2.5). These definitions are not nested inside the capsule's definition as they are generic, independent of the capsule's specific and so they can be defined globally, as is done in Definition 25.

- Each $C m_{j}$ is the name of the process definition for capsule $c_{i}$ such that $m_{j}=\operatorname{name}\left(c_{i}\right)$. This definition is $\mathcal{T}_{C} \llbracket c_{i} \rrbracket \rho$ and is of the form:

$\operatorname{proc} C m_{j}\left(h o o k, p_{1}, p_{1}^{\prime}, p_{2}, p_{2}^{\prime}, \ldots, p_{h}, p_{h}^{\prime}\right.$, ctrl, async $)=\ldots$

In the invocation of $\mathrm{Cm}_{j}$, the actual port sequence arguments $p_{j, 1}, p_{j, 1}^{\prime}, p_{j, 2}, p_{j, 2}^{\prime}, \ldots, p_{j, h}, p_{j, h}^{\prime}$ is such that: - either $p_{j, j^{\prime}} \in R \cup I$ and $l: p_{j, j^{\prime}} \leftrightarrow m_{j} \cdot p_{j^{\prime}} \in K$
- or $p_{j, j^{\prime}}=l \in L$ and $l: m_{j} \cdot p_{j^{\prime}} \leftrightarrow m_{k} \cdot p_{k} \in K$ for some port reference $m_{k} \cdot p_{k}$

- or $p_{j, j^{\prime}}=\operatorname{sink}$ and there is no connector $l$ : $m_{j} \cdot p_{j^{\prime}} \leftrightarrow F \in K$

\section{Explanation}

The Body in the definition of a capsule creates all its parts: 1) an instance of the CapsuleHandler process, 2) an instance of the StateMachine of the capsule, 3) an instance of the WiredEIPort process for each wired end or internal port, 4) an instance of the WiredRPort process for each wired relay port, 5) an instance of the UnwiredEIPort process for each unwired end or internal port, 6) an instance of UnwiredRPort for each unwired relay port, 7) an instance of $C m_{i}$ for each fixed subcapsule $\left.m_{i}, 8\right)$ an instance of $O p t$ for each optional part, and 9) an instance of Plugin for each plug-in part. The arguments of fixed, optional and plugin sub-capsules are such that they correspond to the connectors in the model. Figure 18 shows an overview of the structure of this process.

The Body of the capsule is initialized depending on the value of the parameter async. This boolean parameter specifies whether the capsule is in the same physical thread of its parent (i.e., connected to the same controller) or not. If async is false, the capsule is in the same thread, and Body is executed right away. If not, the capsule is initialized by sending a special "system initialization" event ("sys") to the controller, thus treating capsule initialization as any other event, and passing a dummy completion event $s m c^{\prime}$ to the controller. The capsule will start executing its Body (creating its state machine and sub-capsules) when the controller tells it that it can go ahead and do that (on reception of the message ("sys", "init", null〉).

The last part of the definition of Body holds the instantiation of the CapsuleHandler and StateMachine processes until all sub-capsules have triggered their hook event. This ensures that all sub-capsules are initialized in a bottom-up fashion. (SVP 7) Once all subcapsules have triggered their hook, we instantiate the StateMachine and CapsuleHandler processes and we wait for the state machine to signal on its completion channel smc when it is ready. The smc event is triggered whenever the state machine reaches a stable state, namely when entering a basic state. When this event is received, the capsule can trigger its hook to indicate its readiness to its parent. Furthermore, if it is an asynchronous instantiation, we also trigger the dummy completion event $s m c^{\prime}$ to tell the controller that the capsule has been created.

Definition 17 (Capsule handler) The definition 


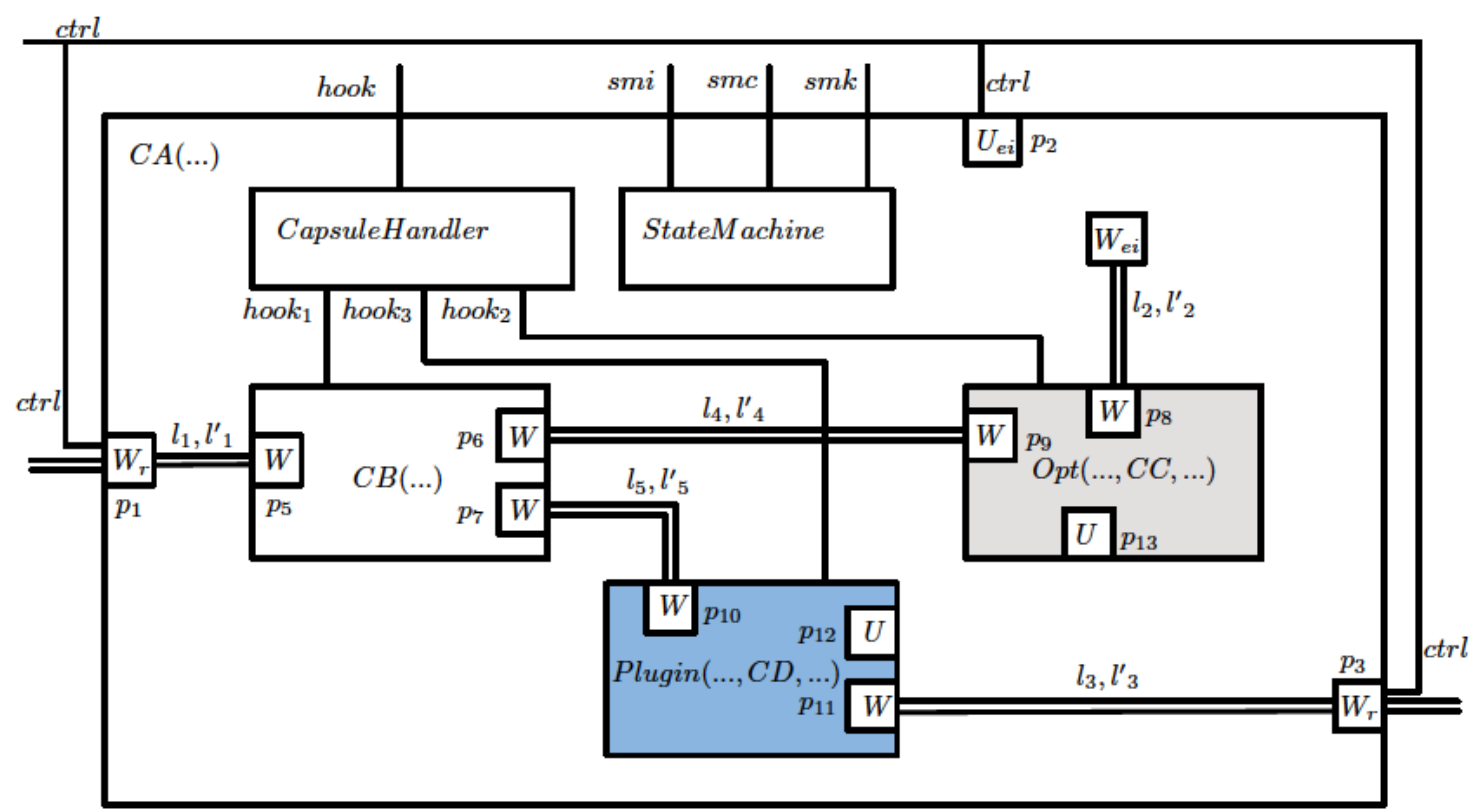

Fig. 18 Overview of the structure of the $\pi_{k l t}$ process of the capsule diagram in Figure 1. Note that connectors are represented as a pair of channels (e.g., $l_{4}, l_{4}^{\prime}$ ). Wired end or internal ports are labelled $W_{e i}$, wired relay ports are labelled $W_{r}$, unwired end or internal ports are labelled $U_{e i}$ and unwired relay ports are labelled $U_{r}$. For sub-capsules $B, C$ and $D$, we do not mark the subscripts $e i$ or $r$, as this is specified internally in those capsules. To keep the diagram simple we have left out several details, such as the port-handler channel $h_{i}$ for each port or the $c t r l$ channel linking each sub-capsule port to their controller.

$D_{\text {CapsuleHandler }}$ of the capsule handler is as follows:

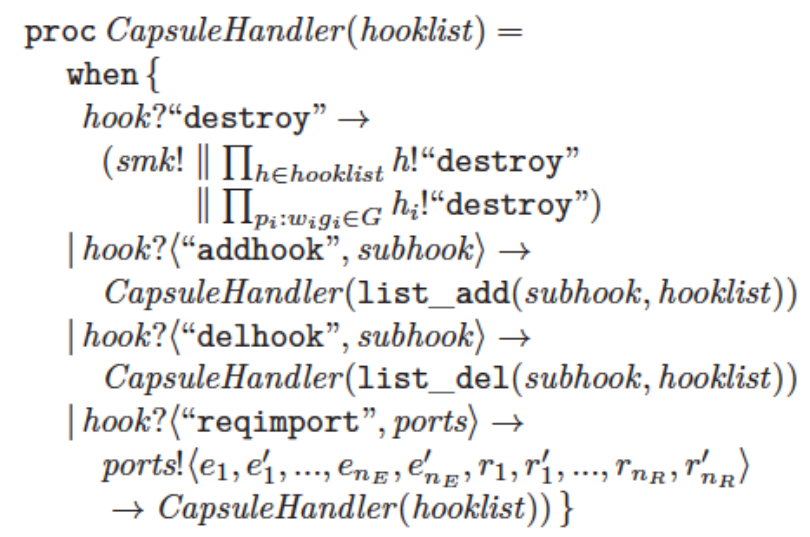

The capsule Handler accepts requests to:

- be destroyed ("destroy"), in which case it sends a kill signal to the state machine $(s m k)$, a "destroy" message to the hook of every sub-capsule and every port,

- add new sub-capsule hooks ("addhook") from an optional part, (see Definition 20 below)

- remove sub-capsule hooks ("delhook"),

- be imported as a plug-in elsewhere ("reqimport"), in which case it answers by providing the links to the capsules external interface.

\subsubsection{Ports and services}

Ports can be wired or unwired. Each port has a "handle" channel $h$ where it receives requests to send messages, or to bind/unbind. It also has a pair of channels $p^{i n}$ and $p^{\text {out }}$ where actual communication of messages happens. In addition, if the port is an end port or an internal port it has a link ctrl to the capsule's controller, $s m i$ and $s m c$ to the capsule's state machine, and the symbolic name of the port pname. If it is a relay port, it a pair of links $q^{\text {in }}, q^{\text {out }}$ to the sub-capsule connected to it.

For a wired end port or internal port, the behaviour is as follows:

- When a message $\langle e, d\rangle$ arrives on the port's $p^{i n}$, a message of the form $\langle s m i, s m c$, pname, $e, d\rangle$ is forwarded to the controller, so that the controller decides when to pass the message to the state machine. This message includes the input port of the state machine $s m i$ and the state machine completion event $s m c$ so that the controller can know where to send the actual message, and wait for the event to be processed fully by the state machine.

- When a "send" request arrives on the handle channel $h$, the message is simply sent through the output port $p^{\text {out }}$.

- When an "unbind" request is received, the process switches to the unwired mode. 


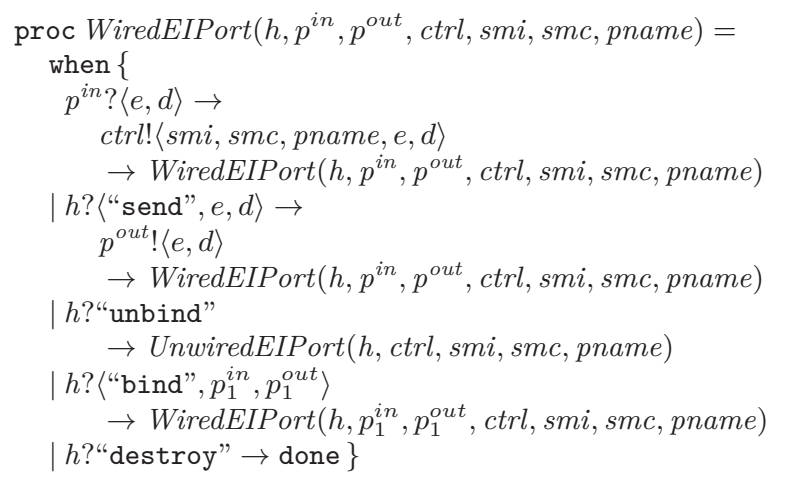

Fig. 19 Wired end or internal ports.

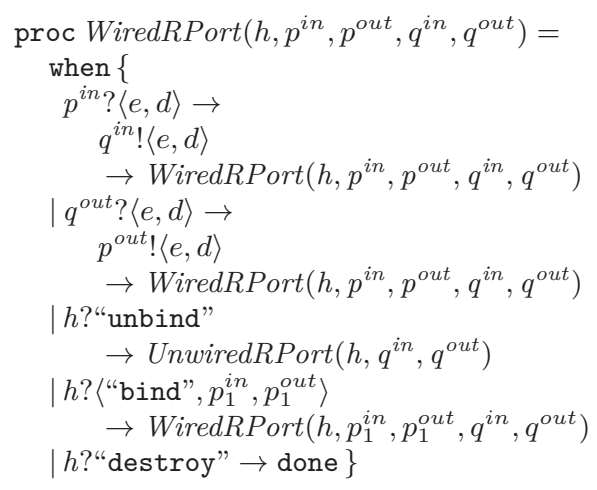

Fig. 20 Wired relay ports.

- When a "bind" message arrives with new channels $p_{1}^{\text {in }}, p_{1}^{\text {out }}$, the process simply replaces the old links with the new ones.

- When a "destroy" message arrives, the process stops.

For a wired relay port, the behaviour is as follows:

- When a message $\langle e, d\rangle$ arrives on the port's $p^{i n}$, it is resent to the sub-capsule though $q^{i n}$.

- When a message arrives on port $q^{\text {out }}$ from a subcapsule, it is resent to the outside through the port $p^{\text {out }}$.

- Messages "bind", "unbind" and "destroy" are handled in the same way as end and internal ports.

For an unwired port, the behaviour is:

- When a "bind" message arrives with new channels $p_{1}^{\text {in }}, p_{1}^{\text {out }}$, the process switches to the wired mode with these new channels as parameters.

- When a "destroy" message arrives, the process stops.

Formally we define these processes below.

Definition 18 (Ports) The definition $D_{\text {WiredEIPort }}$ for wired end or internal ports is given in Figure 19. The definition $D_{\text {WiredRPort }}$ for wired relay ports is given in Figure 20. The definition $D_{\text {UnwiredEIPort }}$ for unwired end or internal ports is given in Figure 21. The definition $D_{\text {UnwiredRPort }}$ for unwired relay ports is given in Figure 22.

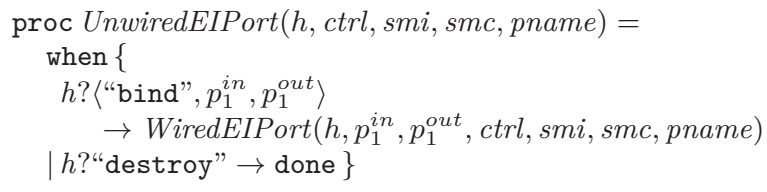

Fig. 21 Unwired end or internal ports.

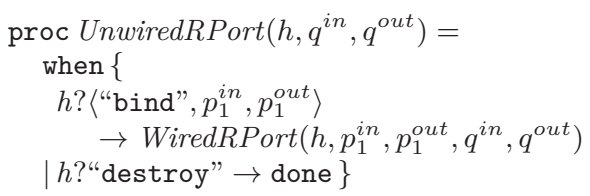

Fig. 22 Unwired relay ports.

The request to send a message may come directly from an action (see Definition 22), and the request to be destroyed may come from the capsule's handler (see Definition 17) but the request to bind or unbind a port always comes from a global "service handler". This is because to bind wired ports a globally unique service name must be provided for ports to be linked: ports can be registered as either service provision points (SPPs) or service access points (SAPs). Connections are established only between SPPs and SAPs: when an SPP is registered under the same service name as some SAP, the two become bound (connected). When an action registers an unwired port, it sends the registration request to the service handler, which keeps track of all registered ports, and links them whenever two ports match the same service name. (SVP 8)

Definition 19 (Service handler) The definition of the service handler process $D_{\text {ServiceHandler }}$ is as shown in Figure 23.

The service handler receives requests in the req port. It keeps two dictionaries spps and saps. These dictionaries are indexed by the service name, and the values are (channels to) port handlers. When a request to register a port as an SPP arrives for a service name sname, the service handler looks up the service name in the saps dictionary. If there was an SAP already there under the service name, it creates a new pair of channels $l, l^{\prime}$ and sends a "bind" message to the port handler of both the SPP and SAP, linking them. After this, the new port handler is added to the spps dictionary. If there was no matching SAP, we only add the new port handler spps. A request to register a port as an SAP is symmetric. Deregistering is achieved in a similar fashion, sending "unbind" messages to the corresponding ports.

\subsubsection{Optional and plug-in parts}

Definition 20 (Optional Parts) The definition $D_{O p t}$ of the optional part handler process is defined as shown in Figure 24. 


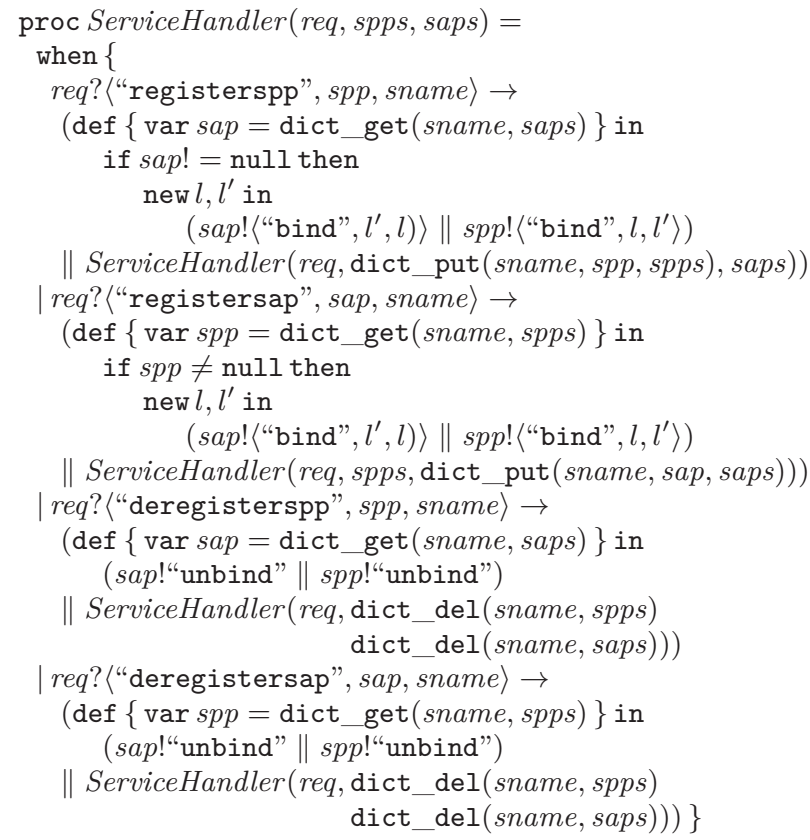

Fig. 23 Service handler

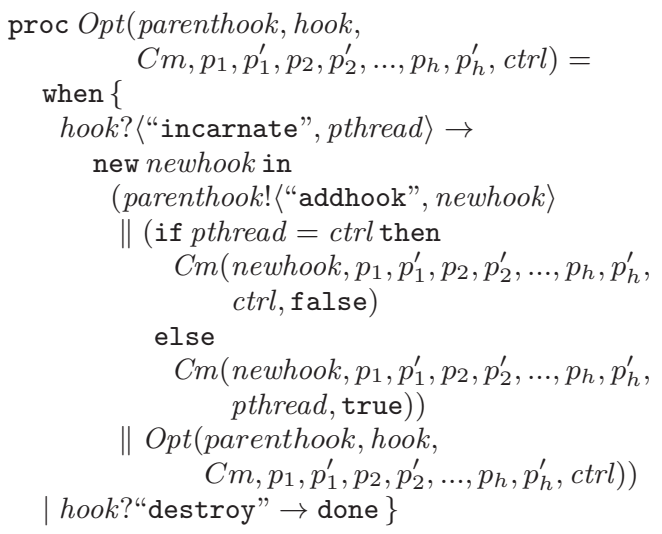

Fig. 24 Optional parts.

In this definition, an optional part acts as a placeholder, which can receive requests to incarnate a capsule $\mathrm{Cm}$ in some thread $t$ (or in the same thread as its parent, if pthread is $c t r l$ ). When a request to incarnate a new instance arrives, a message is sent to the containing capsule's handler to add the new $C m$ is instantiated with the proper connections. When a request to be destroyed arrives, the part simply stops. In this case, the parent's capsule handler takes care of destroying all created instances. This destroys only the part, not the capsules incarnated in it. Their destruction is addressed by the capsule handler itself.

Definition 21 (Plug-in Parts) The following auxiliary process is used to connect ports:

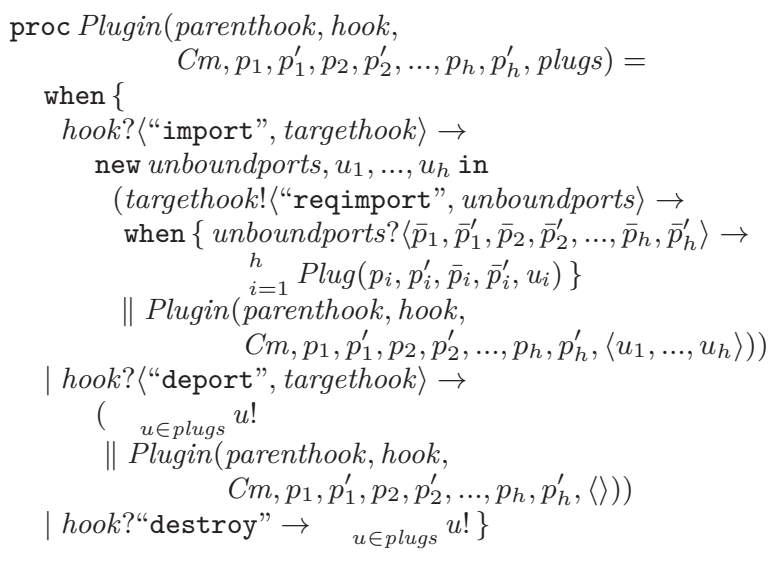

Fig. 25 Plugin parts.

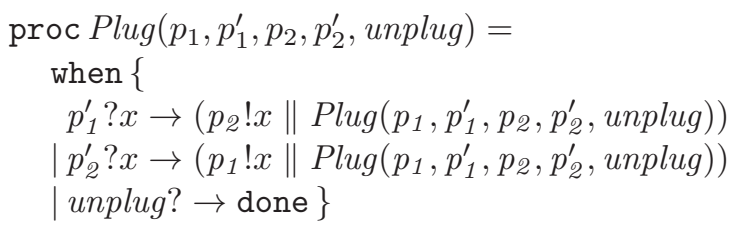

The definition $D_{\text {Plugin }}$ of the plug-in part handler process is defined as shown in Figure 25.

The Plug process simply binds two port pairs, by acting as a message forwarder, connecting the output $p_{1}^{\prime}$ to the input $p_{2}$ and the output $p_{2}^{\prime}$ to the input $p_{2}$. The Plugin waits for import requests which come with the imported (targethook). The plug-in part asks that capsule to provide a list of its (unbounded) ports to be bound. When the answer arrives, these ports are bound by the Plug instances. The plugs parameter keeps a list of the unplug port for each plug. When a "deport" or "destroy" message arrives a signal is sent to unplug all plugs.

Example 6 Let us revisit the capsule from Figure 1 and Example 5. Figure 26 shows the (top-level) translation of this capsule. See also Figure 18.

Note that the order of ports in the definition is such that end-ports $\left(p_{2}\right)$ go first and they are followed by relay ports $\left(p_{1}\right.$ and $\left.p_{3}\right)$. We list the connectors for internal ports $\left(l_{2}, l_{2}^{\prime}\right)$ before the rest. The process $C B$ is the process defined for capsule $B$. Similarly for capsules $C$ and $D$. Their definitions will be of the form:

$$
\begin{aligned}
& \operatorname{proc} C B\left(\text { hook }, p_{5}, p_{5}^{\prime}, p_{6}, p_{6}^{\prime}, p_{7}, p_{7}^{\prime}, \text { ctrl }\right)=\ldots \\
& \text { proc } C C\left(\text { hook }, p_{8}, p_{8}^{\prime}, p_{9}, p_{9}^{\prime}, p_{13}, p_{13}^{\prime} \text { ctrl }\right)=\ldots \\
& \operatorname{proc} C D\left(\text { hook }, p_{10}, p_{10}^{\prime}, p_{11}, p_{11}^{\prime}, p_{12}, p_{12}^{\prime}, \text { ctrl }\right)=\ldots
\end{aligned}
$$

Note how the ports and links passed to the process invocation $C B$ (in Body) correspond to the (positional) parameters of its definition according to the connections in the diagram. So for example, parameter $p_{5}$ of $C B$ receives as argument $p_{1}$ since there is a relay link $l_{1}$ 


$$
\begin{aligned}
& \mathcal{T}_{C} \llbracket c_{1} \rrbracket \theta \stackrel{\text { def }}{=} \\
& \text { proc } C A\left(h o o k, p_{2}, p_{2}^{\prime}, p_{1}, p_{1}^{\prime}, p_{3}, p_{3}^{\prime}, \text { ctrl }\right)= \\
& \text { new } s m i, s m c, s m k \text {, } \\
& l_{2}, l_{2}^{\prime}, \\
& l_{1}, l_{1}^{\prime}, l_{3}, l_{3}^{\prime}, l_{4}, l_{4}^{\prime}, l_{5}, l_{5}^{\prime} \text {, } \\
& h_{1}, h_{2}, h_{3}, h_{4} \text {, } \\
& \text { hook }_{1} \text {, hook } 2 \text {, hook } 3 \text { in } \\
& \operatorname{def}\left\{D_{\text {StateMachine }} ; D_{\text {CapsuleHandler }} ; D_{\text {Body }}\right\} \text { in } \\
& \text { if async then } \\
& \text { new } s m c^{\prime} \text { in } \\
& c t r l !\langle s m i, s m c \text {, "sys", "init", null }\rangle \rightarrow \\
& \text { when }\{\text { smi? }\langle\text { "sys", "init", null〉 } \rightarrow \\
& \text { else Body (smc, false) } \\
& \left.\operatorname{Body}\left(s m c^{\prime}, \text { true }\right)\right\}
\end{aligned}
$$

where Body is

$$
\begin{aligned}
& \operatorname{proc} \operatorname{Body}\left(s m c^{\prime}, \text { async }\right)= \\
& \text { WiredRPort }\left(h_{1}, p_{1}, p_{1}^{\prime}, l_{1}, l_{1}^{\prime}\right) \\
& \text { \|WiredEIPort }\left(h_{4}, l_{2}, l_{2}^{\prime}, c t r l, \text { smi, smc, " } \mathrm{p}_{4}\right. \text { ") } \\
& \text { \| UnwiredEIPort ( } h_{2}, p_{2}, p_{2}^{\prime}, \text { ctrl, smi, smc, " } \mathrm{p}_{2} \text { ") } \\
& \text { \|UnwiredRPort }\left(h_{3}, p_{3}, p_{3}^{\prime}, l_{3}, l_{3}^{\prime}\right) \\
& \| C B\left(h_{o o k_{1}}, l_{1}, l_{1}^{\prime}, l_{4}, l_{4}^{\prime}, l_{5}, l_{5}^{\prime}, \theta(B), \mathrm{false}\right) \\
& \| \operatorname{Opt}\left(\text { hook, hook }, C C, l_{2}, l_{2}^{\prime}, l_{4}, l_{4}^{\prime}, \text { ctrl }\right) \\
& \text { \| Plugin (hook, hook } \left.{ }_{3}, C D, l_{5}, l_{5}^{\prime}, p_{3}, p_{3}^{\prime},\langle\rangle\right) \\
& \| \text { when }\left\{\left\langle\text { hook }_{1}, \text { hook }_{2}, \text { hook }_{3}\right\rangle \text { ? } \rightarrow\right. \\
& \text { (CapsuleHandler }\left(\left\langle\text { hook }_{1}, \text { hook }_{2}, \text { hook }_{3}\right\rangle\right) \\
& \text { \| StateMachine (smi, smc, smk, } \left.p_{2}^{\prime}, l_{2}^{\prime}\right) \\
& \left.\| \text { when }\left\{s m c \text { ? } \rightarrow \text { hook! } \rightarrow \text { if async then } s m c^{\prime} !\right\}\right)
\end{aligned}
$$

Fig. 26 Translation of capsule $c_{1}$ from Example 5 .

between them, and parameter $p_{6}$ receives $l_{4}$, the local channel that represents the connector with the same name. The same applies to the optional and plug-in parts.

The hook ${ }_{1}$ links $C A$ 's capsule handler and state machine with sub-capsule $B$. Similarly, hook $k_{2}$ is used to interact with part $C$ and $h_{0 o k_{3}}$ to interact with part D.

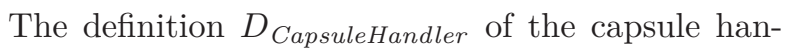
dler is as follows:

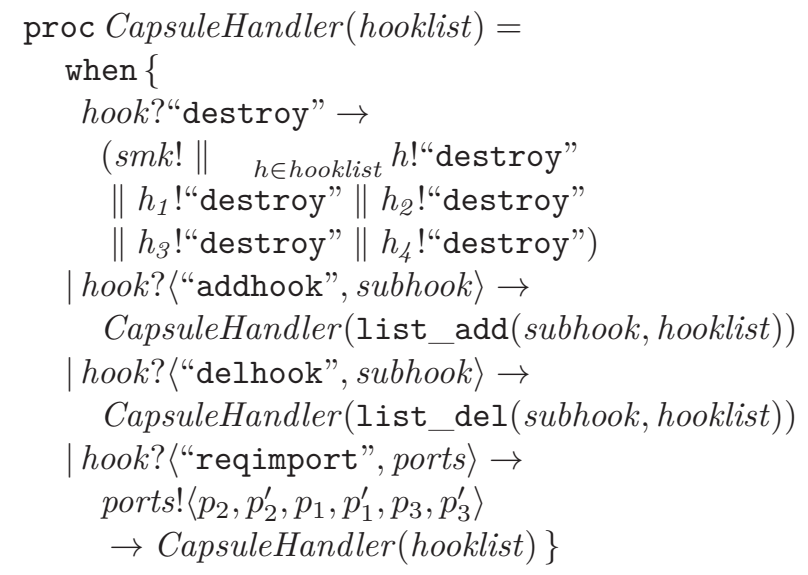

\subsubsection{Translating actions}

We now present the translation $\alpha$ for the action language from Definition 12.

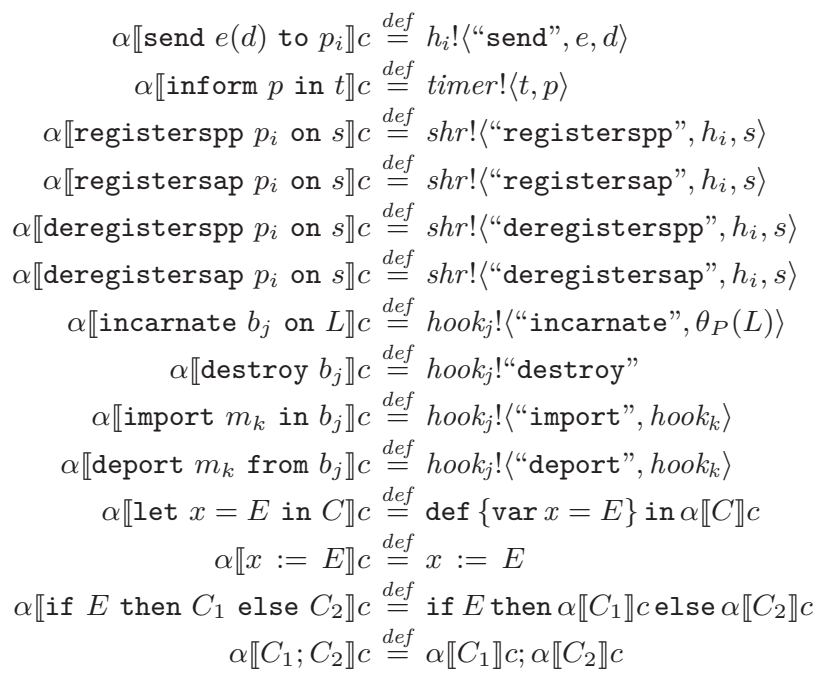

Fig. 27 Mapping actions

Definition 22 (Actions to processes) We define the map $\alpha:$ Acts $\rightarrow \mathcal{C} \rightarrow$ KLT, where Acts is the action language from Definition 12, with context set $\mathcal{C}$ whose elements are triples $\langle\langle p, e, d\rangle$, ports, $\theta\rangle$ of incoming events, lists of ports and thread assignment as shown in Figure 27. In this definition:

- in the case of inform, the channel timer is the global channel to request a timeout event from the Timer process (Definition 23) declared in Definition 25,

- in the cases for send and inform as well as for the sap/spp (de)registering operations, $h_{i}$ is the name of the handle channel for port $p_{i}$, and $s h r$ is the (global) request channel for the ServiceHandler (Definition 19), declared at the top level (Definition 25).

- in the case for incarnate, $h o o k_{j}$ is the name of the channel corresponding to part $b_{j}$ and

- in the cases for import and deport, hook $k_{k}$ is the $m_{k}$ and $h o o k_{j}$ is the name of the channel corresponding to part $b_{j}$. Note that we assume that $t$ is the name of a channel which corresponds to a physical thread (see Definition 25 below).

The actions corresponding to local variables, assignment, conditionals and sequential composition are translated directly into their corresponding constructs in kiltera. Note however, that these constructs can themselves be expressed purely in terms of the other constructs. Here we do not elaborate on such encoding, as it falls beyond the scope of this paper.

\subsubsection{The timer}

The timer process accepts requests to schedule timeout signals on a given port. When it receives a request 
$(t, p)$, it will schedule an event trigger on $p$ after a delay $t$. This is done asynchronously so that multiple capsules/threads can make such scheduling requests without blocking or delaying each other.

Definition 23 (Timer) The definition $D_{\text {Timer }}$ of the timer process is as follows:

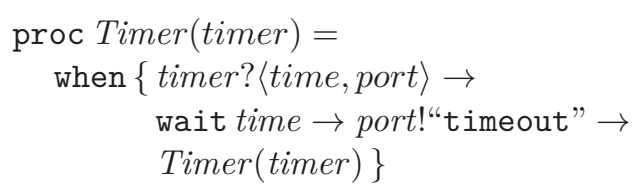

\subsubsection{The full system}

The meaning and behaviour of a UML-RT model depends on the assignment of capsules to threads (and therefore to controllers). Thus the input of the translator must include:

- The UML-RT model (the top-level capsule, including the definitions of all capsules and state machines): a CAP element.

- The maps from capsules to logical-threads and to physical threads: the pair of maps $\theta_{L}$ and $\theta_{P}$.

The kiltera process simulates the entire model by collecting all capsule definitions, and instantiating the top capsule and the controllers, with one controller for each thread.

Definition 24 (UML-RT configuration) A $U M L$ $R T$ configuration is a tuple $\left(U, N_{L}, N_{P}, \theta_{L}, \theta_{P}\right)$ where

- $U \in$ UMLRT, i.e., $U=\left[c_{0}, c_{1}, \ldots, c_{n}\right]$

- $c_{0} \in \mathbf{C A P}$ is $U$ 's top-level capsule term,

- $N_{L} \subseteq \mathcal{N}_{\text {lthr }}$ is a set of logical-thread names,

$-N_{P} \subseteq \mathcal{N}_{p t h r}$ is a set of physical-thread names,

$-\theta_{L}: N_{C} \rightarrow N_{L}$ is a capsule-to-logical-thread assignment where $N_{C} \stackrel{\text { def }}{=}\{$ name $(c) \mid c \in U\}$ is the set of names of all capsules in the model,

- and $\theta_{P}: N_{L} \rightarrow N_{P}$ is a logical-to-physical-thread assignment.

We call UMLRTC the set of all possible UML-RT configurations.

Now we can provide the translation of a full input model. We create a sink to serve as sink for state machine events, an event $s h r$ where the service handler will receive requests, an event timer where timer will receive requests, a channel tophook to serve as the hook channel for the top-level capsule, and an event/channel $T_{i}$ for each thread, which will be specific to each controller. The main construction simply creates an instance of the service handler, of the timer, the controllers (one for each thread) and the top-level capsule, which in turn will instantiate its sub-capsules.
Definition 25 (Translation of a full configuration) Given a UML-RT configuration $M=\left(U, N_{L}, N_{P}, \theta_{L}, \theta_{P}\right)$ with a model $U=\left[c_{0}, \ldots, c_{k}\right] \in$ UMLRT, and $N_{P}=$ $\left\{T_{1}, T_{2}, \ldots, T_{n}\right\}$ the set of physical thread names, the translation of $M$ is $\mathcal{M} \llbracket M \rrbracket$ where the function $\mathcal{M} \llbracket \cdot \rrbracket$ : UMLRTC $\rightarrow$ KLT is defined as follows:

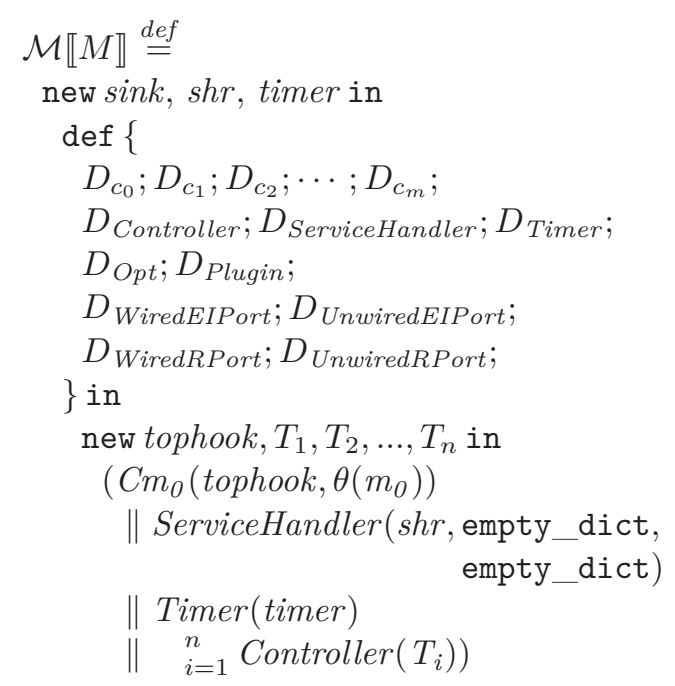

where $m_{0}=\operatorname{name}\left(c_{0}\right)$ is the top-capsule's name, $\theta \stackrel{\text { def }}{=}$ $\theta_{P} \circ \theta_{L}$ is the capsule-to-thread assignment,

$D_{c_{i}} \stackrel{\text { def }}{=} \mathcal{T}_{C} \llbracket c_{i} \rrbracket \theta$

is the translation of capsule $c_{i}$ (see Definition 16) and where $D_{\text {Controller }}$ is the definition of Controller (see Definition 15), $D_{\text {ServiceHandler }}$ is the definition of the ServiceHandler process from Definition $19, D_{\text {Timer }}$ is the definition of Timer from Definition 23, $D_{O p t}$ is the definition of $O p t$ (see Definition 20), $D_{\text {Plugin }}$ is the definition of Plugin (see Definition 21), and $D_{\text {WiredEIPort }}$ and $D_{\text {UnwiredEIPort }}$ are the definitions of the processes for port-handling given in Definition 18. .

Example 7 Let us finish by revisiting Example 1. Assume that $c_{0}, c_{1}, c_{2}$ are the representations of capsules $A, B$ and $C$, and $L_{i}$ and $T_{i}$ are the names of logical and physical threads. The model, or more precisely, the configuration with all capsules mapped to the same physical thread is

$$
\begin{aligned}
& M_{0}=\left(\left\{c_{0}, c_{1}, c_{2}\right\},\left\{L_{0}, L_{1}\right\},\left\{T_{0}, T_{1}\right\},\right. \\
& \left.\quad\left\{A \mapsto L_{0}, B \mapsto L_{0}, C \mapsto L_{1}\right\},\left\{L_{0} \mapsto T_{0}, L_{1} \mapsto T_{0}\right\}\right)
\end{aligned}
$$

and the configuration with capsule $C$ mapped to a different physical thread is

$$
\begin{aligned}
& M_{1}=\left(\left\{c_{0}, c_{1}, c_{2}\right\},\left\{L_{0}, L_{1}\right\},\left\{T_{0}, T_{1}\right\},\right. \\
& \left.\quad\left\{A \mapsto L_{0}, B \mapsto L_{0}, C \mapsto L_{1}\right\},\left\{L_{0} \mapsto T_{0}, L_{1} \mapsto T_{1}\right\}\right)
\end{aligned}
$$




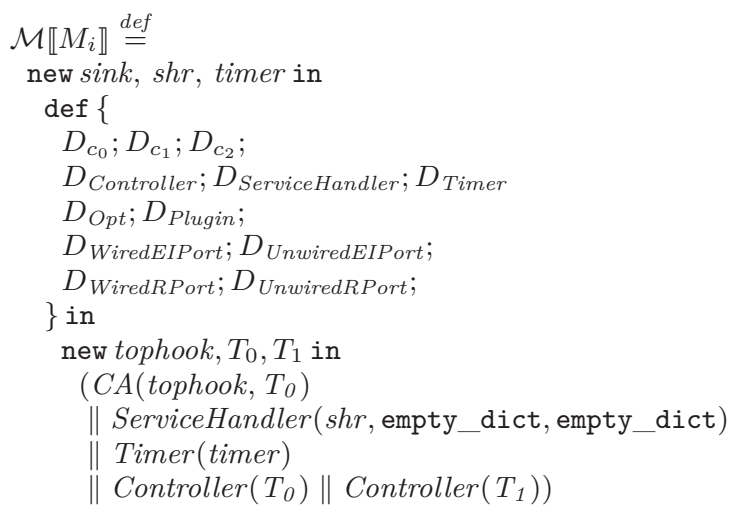

Fig. 28 Generated $\pi_{k l t}$ model for $M_{i}$ (Example 1 with two physical threads).

In both cases $C$ is associated with logical thread $L_{1}$ but in the first, $L_{1}$ is assigned to physical thread $T_{0}$ (the same as $A$ and $B$ ) whereas in the second it is assigned to $T_{1}$. Then, the resulting $\pi_{k l t}$ according to Definition 25, is shown in Figure 28. So while in both cases we have two physical threads (and two controllers), the second controller is used only in $M_{1}$. More precisely, the incarnate action in state $n_{2}$ of $A$ (incarnate $C$ on $L_{1}$ ) is translated according to Definition 22 into the term

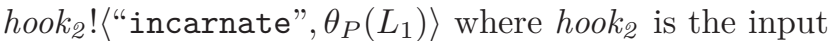
channel for $C C$ 's control handler. This means that for $M_{0}$ this action is hook ! "incarnate", $T_{0}$ ) and for $M_{1}$ it is hook ${ }_{2}$ ! "incarnate", $\left.T_{1}\right\rangle$. Hence, according to Definition 20, when the Opt process inside the top-level capsule $C A$ receives this incarnation message, it will create a newhook link to the new capsule instance and send an 〈"addhook", newhook〉 message to $C A$ 's capsule handler. Then it will invoke the process $C C$ to instantiate the new capsule, but in the first case the invocation will be $C C$ (newhook, $p_{4}, p_{4}^{\prime}, T_{0}$, false) whereas in the second case it will be $C C$ (newhook, $p_{4}, p_{4}^{\prime}, T_{1}$, true). As a result, in the first case the instance of $C C$ will communicate with the controller on $T_{0}$ thus sharing the same event pool with $C A$ and $C B$, and in the second, with the controller on $T_{1}$ with its own separate event pool. We could modify the example to have only one physical thread and one or more logical threads with the same effects.

\section{Related work}

There have been many approaches proposed in the literature, aiming to formalize different aspects of UML. For example, [13] proposes a semantics of activity diagrams using labelled transition systems, while [59] presents a semantics of activity diagrams using Petri Nets. In [32] a semantics of sequence diagrams is proposed in terms of certain kind of transition system, while [8] uses a custom temporal logic for defining the semantics of sequence diagrams. Aspects of UML state machines have been formalized, amongst others, in [33] using Generalized Stochastic Petri Nets, in [45] using term rewriting systems, in [39] and in [62] using CSP, or in [37] using LOTOS. A semantics for a kernel action language for UML has been proposed in [14] using labelled transition systems.

Other related work includes [9] where a subset of the UML for real-time systems called krtUML is proposed and its semantics formalized with symbolic transition systems. In [58], a semantics is presented for a subset of UML consisting of flat state machines and sequence diagrams with no hierarchical structure diagrams using linear temporal logic. [36] studies a real-time extension of UML state machines providing a semantics in terms of timed-automata. In [65] a semantics for a real-time variant of standard UML state machines is presented in terms of transition systems.

The book UML 2 Semantics and Applications [30] includes several articles proposing formal semantics for fragments of UML 2, including non-flattening semantics for state machines. Nevertheless, UML-RT itself is not the same as UML or real-time UML, and work formalizing it is less common.

A number of papers have presented formal semantics for small subsets of UML-RT using either CSP or some timed variant of CSP $[16,12,15,7,1]$.

In [16], only capsule diagrams are translated into CSP processes, assuming synchronous communication (the default in CSP), no state machines, and no support for dynamic features such as optional or plug-in capsules, dynamic wiring or thread assignments.

In [12], a translation to CSP is also provided with the aim of studying the preservation of consistency in model evolution. This translation deals only with flat state machines and flat structure diagrams and no dynamic structure features or thread assignments.

The translation in [15] actually goes in the opposite direction, from CSP processes to UML-RT.

In [7], and later in [1], a transformation of UML-RT models into a timed variant of CSP called CSP $+\mathrm{T}$ is proposed. It addresses hierarchical state machines but without group transitions or history, and like the previous papers, it relies on CSP's synchronous communication and has no support for dynamic features or thread assignment.

A similar approach has been proposed in [52] where the target language is Circus, a combination of CSP and Z. This translation suffers from the same limitations of the previous ones. 
It should be noted that all existing approaches to the semantics of UML-RT state machines, unlike ours, flatten the state machine. This forgets the hierarchical structure, which in turn means that there is no obvious way to encode the priority of inner transitions over outer transitions. Furthermore it complicates traceability between model elements and the generated artifact.

A very different approach is presented in [18] where the semantics of a very small subset of UML-RT is described as an algebra of flow-graphs. This is an interesting approach, but not only is it limited in its scope and coverage of UML-RT but it is also unclear how it could be leveraged for analysis.

The work most closely related to our own is that of [61], [31] and [5,4]. Table 1 summarizes the main differences between these and our semantics.

In [61] a formal semantics for a sub-set of UMLRT is presented using Structural Operational Semantics (SOS) to define a labelled-transition system (LTS) as the semantic domain. This has the advantage that the meta-theory for SOS over LTSs is well developed. On the other hand it does not deal with many essential aspects of UML-RT such as optional and plug-in capsules, dynamic wiring or capsule-to-thread assignments, and no action language is given. Furthermore that paper also distinguishes between basic capsules (without subcapsules), non-behavioural capsules (without a state machine) and behavioural capsules, whereas we do not make such a distinction and the three cases are treated uniformly.

In $[5,4]$ a semantics for UML-RT is proposed using the $\pi$-calculus. However this considers only a very small subset of UML-RT, without hierarchical state machines, no group transitions or history, a very limited form of rewiring, no optional or plug-in capsules, no threads or controllers, and no attributes. Furthermore, the presentation of this semantic mapping is by example only, without an actual formal mapping or other systematic way of translating models into $\pi$-calculus terms.

The work in [31] is much more elaborate with respect to UML-RT than any other attempt. The authors propose a semantics for UML-RT in terms of AsmL, an object-oriented language based on Abstract State Machines (ASMs). They propose an architecture to support alternative semantics for UML-RT by relying on object-oriented polymorphism in AsmL. Different UML-RT concepts are represented as AsmL classes. Unlike all previous papers they support optional and plug-in classes as well as multiple controllers. However they only support flat state machines and no dynamic wiring of SPPs and SAPs. Furthermore they do not provide an automatic translation of UML-RT models so the modeller must manually represent the model as an AsmL data-structure.

Finally we cite our previous work [10] where we introduced early version of the mapping of state machines without history or enabled-transition selection policy, and without support for capsules.

\section{Concluding remarks}

We have proposed a formal syntax and semantics for the UML-RT language in terms of a process algebra called kiltera. We believe this is the most comprehensive formalization of the semantics of UML-RT to date. Unlike existing attempts, our formalization deals with both fully hierarchical state machines and structure diagrams. On both aspects it supports features not available in other approaches, such as history or group transitions in state machines, or optional capsules and dynamic wiring in capsule diagrams. It is the only semantics with explicit support for thread assignment.

In addition to these contributions, it should also be noted that unlike much of the existing approaches, we provide an actual mapping specifying the translation, whereas some papers simply propose their semantics in an ad hoc by-example manner, without providing an actual translation. Furthermore, our mapping has been implemented using IBM RSA's transformation tool, providing a realization of the semantics. The outcome of this translation can be used by the implementation of kiltera for simulation. The development of this implementation itself helped validating the translation. We are currently working towards a kiltera modelchecker which will provide analysis capabilities. The modular nature of the translation, including mapping of states and capsules to processes can be leveraged by the model-checker for the purpose of traceability, by providing a simple way to link the results of analysis on the generated kiltera code to the corresponding model elements.

Semantic variation points are an issue whenever we attempt to formalize a language for which the semantics has been only partially given. This is the case with the UML in general and with UML-RT in particular. We have attempted to define our mapping as precise as possible while marking semantic variation points explicitly. Nevertheless it is important to keep in mind that there are different kinds of variation points, some of which can be easily addressed and some which would require major changes in the mapping. For example, the action language can be changed or extended with relative ease simply by providing an alternative mapping $\alpha$ to be invoked by the translation of state machines in Definition 6 and Definition 7. The transla- 


\begin{tabular}{|l|l|l|l|l|l|}
\hline & Features & {$[61]$} & {$[31]$} & {$[5,4]$} & Ours \\
\hline \hline Underlying semantics & & LTS & AsmL & $\pi$-calculus & $\pi_{k l t}$-calculus \\
\hline Semantics definition & & SOS & Hard coded & By example & By translation \\
\hline Executable & & No & Yes & No & Yes \\
\hline State Machines & Hierarchy & Yes & No & No & Yes \\
\hline & Group transitions & Yes & No & No & Yes \\
\hline & Deep history & Yes & No & No & Yes \\
\hline & Enabled transition selection & Yes & No & No & Yes \\
\hline Action language & & No & Subset & Subset & Subset \\
\hline Timer service & & No & No & No & Yes \\
\hline Capsules & Fixed capsules & Yes & Yes & Yes & Yes \\
\hline & Optional capsules & No & Yes & No & Yes \\
\hline & Plugin capsules & No & Yes & No & Yes \\
\hline & Attributes & No & Yes & No & Yes \\
\hline & Services & No & No & No & Yes \\
\hline & Dynamic wiring & No & Yes & No & Yes \\
\hline & Capsule-thread assignment & No & Yes & No & Yes \\
\hline & Multiple controllers & No & Yes & No & Yes \\
\hline
\end{tabular}

Table 1 Comparison of formal semantics for UML-RT.

tion of state machines themselves can be replaced in its entirety by providing an alternative definition of $\mathcal{T}_{S M}$ (Definition 8) which is invoked by the translation of capsules in Definition 16. However, some semantic variation points require more delicate "surgery". For example changing the enabled-transition selection policy from inside-out to outside-in would require replacing the process Handler in the definition for composite states Definition 7. Changing it to full non-determinism would imply an even more radical change, even eliminating the need for the accept/reject protocol. Similarly in the mapping of capsules (Definition 16), details of the difference between incarnation in the same thread or in a different thread, or the bottom-up initialization of capsules could be changed by an alternative definition of $\mathcal{T}_{C}$.

One aspect that we did not touch was the encoding of protocols. This is because our mapping encodes the dynamic behaviour of UML-RT, while protocols (in UML-RT) contain only static type information, specifically the type of events allowed in a given port. In other words, our mapping assumes that the input model is well-typed, and under such assumption, it will give the model's behaviour.

We have proposed a semantics of UML-RT by means of translation to another language with a well defined formal semantics. In the introduction we motivated our choice of kiltera as the target language on the basis of its conceptual similarities to UML-RT. Nevertheless, in spite of these similarities, the mapping is not trivial. This observation is important because most of the existing work on formalizing rich, expressive and realistic languages tends to oversimplify them, overlooking many aspects that are often deemed "irrelevant" or "implementation-specific" but turn out to be seman- tically meaningful, as is the case with thread assignments in UML-RT. A common mistake is to assume that similar concepts are mapped in a one-to-one fashion between the source and the target language. But the thread assignment issue illustrates the problem. For example, in $[5,4]$, capsules are mapped onto $\pi$-calculus processes without regard for their thread assignment. This would be fine but only under the assumption that each capsule executes on a separate physical thread. Otherwise, analysis of the resulting $\pi$-calculus processes would fail to detect possibilities for deadlock, or behaviours depending on message ordering. In short, it would lead to incorrect analysis results. This highlights the perils of using minimalistic and pure languages or formalisms to define semantics of realistic languages.

Artifacts Our kiltera simulator prototype is available at http://www.kiltera.org. Some sample UML-RT models (for IBM's RSA-RTE) together with their generated kiltera code and their meta-models are available at the Repository for Model-Driven Development (ReMoDD: http://www.cs.colostate.edu/remodd/v1/). The actual transformation is available directly from the authors.

Acknowledgements We are indebted to Bran Selic for his support and his insights into the semantics of UML-RT. We also thank Malina Software, IBM Canada and the Natural Sciences and Engineering Research Council of Canada (NSERC) and the Ontario Centres for Excellence (OCE) which provided financial support. We would also like to thank Eyrak Paen for his implementation of the transformation which was instrumental in the proper definition of the mapping. Finally, Karolina Zurowska and Eric Rapos also provided very useful input on RoseRT and RSA-RTE. 


\section{References}

1. K. Benghazi Akhlaki, M.I. Capel Tuñón, J.A. Holgado Terriza, and L.E. Mendoza Morales. A methodological approach to the formal specification of real-time systems by transformation of UML-RT design models. Science of Computer Programming, (65):41-56, 2007.

2. J. A. Bergstra and J. W. Klop. Process algebra for synchronous communication. Information and Control, 60(13):109-137, 1984.

3. A. Bertolino, G. De Angelis, C. Bartolini, and G. Lipari. A UML profile and a methodology for real-time systems design. Technical report, Istituto di Scienza e Tecnologie dell'Informazione "A. Faedo", 2005.

4. J. de M. Bezerra and C. M. Hirata. A Semantics for UMLRT using $\pi$-calculus. In Proc. of Int. Workshop on Rapid System Prototyping (RSP'07), 2007.

5. J. de M. Bezerra and C. M. Hirata. A polyadic pi-calculus approach for the formal specification of UML-RT. Adv. Software Engineering, 2009, 2009.

6. G. Boudol. Asynchrony and the $\pi$-calculus (Note). Technical Report 1702, INRIA-Sophia Antipolis, 1992.

7. M. I. Capel, L. E. Mendoza, K. B. Akhlaki, and J. A. Holgado. A semantic formalization of UML-RT models with $\mathrm{CSP}+\mathrm{T}$ processes applicable to real-time systems verification. In Proc. of Jornadas de Ingeniería del Software y Bases de Datos (JISBD'06), pages 283-292, 2006.

8. S. M. Cho, H-H. Kim, S.D. Cha, and D-H. Bae. A semantics of sequence diagrams. Information Processing Letters, 84(3):125-130, 2002.

9. W. Damm, B. Josko, A. Pnueli, and A. Votintseva. Understanding UML: A Formal Semantics of Concurrency and Communication in Real-Time UML. In Proc. of FMCO'02, LNCS, pages 71-98. Springer Verlag, 2002.

10. J. Dingel, E. Paen, E. Posse, R. Rahman, and K. Zurowska. Definition and implementation of a semantic mapping for UML-RT using a timed pi-calculus. In Proc. of the Second International Workshop on Behaviour Modelling: Foundation and Applications, BM-FA '10, pages 1:1-1:8, New York, NY, USA, 2010. ACM.

11. B. P. Douglass. Real-Time UML. In Formal Techniques in Real-Time and Fault-Tolerant Systems, volume 2469 of $L N C S$, pages 53-70. Springer, 2002.

12. G. Engels, R. Heckel, J. M. Küster, and L. Groenewegen. Consistency-preserving model evolution through transformations. In Proc. Fifth International Conference on the Unified Modeling Language - The Language and its Applications, pages 212-227. Springer, 2002.

13. R. Eshuis and R. Wieringa. A Formal Semantics for UML Activity Diagrams - Formalising Workflow Models. Technical report, University of Twente, 2001.

14. H. Fecher, M. Kyas, W-P. De Roever, and F. S. De Boer. Compositional Operational Semantics of a UML-KernelModel Language. Electronic Notes in Theoretical Computer Science, (156):79 - 96, 2006.

15. P. Ferreira, A. Sampaio, and A. Mota. Viewing CSP Specifications with UML-RT Diagrams. Electronic Notes in Theoretical Computer Science, 195(0):57-74, 2008. Proc. of the Brazilian Symposium on Formal Methods (SBMF 2006).

16. C. Fischer, E.-R. Olderog, and H. Wehrheim. A CSP view on UML-RT structure diagrams. In Proc. Fundamental Approaches to Software Engineering (FASE'01), volume 2029 of $L N C S$, pages 91-108. Springer, 2001.

17. D. Garlan, R. T. Monroe, and D. Wile. Acme: Architectural Description of Component-Based Systems. In Gary T. Leavens and Murali Sitaraman, editors, Foundations of
Component-Based Systems, chapter 3, pages 47-67. Cambridge University Press, New York, NY, 2000.

18. R. Grosu, M. Broy, B. Selic, and G. Stefanescu. Behavioral Specifications of Businesses and Systems, chapter 6: What is behind UML-RT?, pages 73-88. Kluwer Academic Publishers, 1999.

19. C. A. R. Hoare. Communicating Sequential Processes. Comm. of the ACM, 21(8):666-677, August 1978.

20. K. Honda and M. Tokoro. An object calculus for asynchronous communication. In Proc. of ECOOP '91, volume 512 of $L N C S$, pages 133 - 147. Springer, 1991.

21. IBM. General Description Language. IBM, 9 March 2005.

22. IBM. IBM Rational Rose Technical Developer, Version 7.0. IBM, 2010. http://www-01.ibm.com/software/ awdtools/developer/technical.

23. IBM. IBM Rational Software Architect, RealTime Edition, Version 7.5.2. IBM, 2010. http://publib.boulder.ibm. com/infocenter/rsarthlp/v7r5m1/index.jsp.

24. IEEE Computer Society. IEEE Standard Verilog ${ }^{\circledR}$ Hardware Description Language, IEEE Standard $1364^{\text {TM }}-2001$, 28 September 2001.

25. IEEE Computer Society. IEEE Standard VHDL Language Reference Manual, IEEE Standard $1076^{\mathrm{TM}}-2008,26$ January 2009.

26. IEEE Computer Society. IEEE Standard for the SystemC Language, IEEE Standard $1666^{\mathrm{TM}}-2011$, January 2012.

27. IEEE Computer Society. IEEE Standard for SystemVerilog - Unified Hardware Design, Specification, and Verification Language, IEEE Standard 1800 ${ }^{\mathrm{TM}}-2012,21$ February 2013.

28. International Telecommunications Union. Specification and description language (SDL). ITU-T Recommendation Z.100., November 1999.

29. D. R. Jefferson. Virtual Time. ACM-TOPLAS, 7(3):404425, July 1985.

30. K. Lano and D. Clark. UML 2 Semantics and Applications, chapter Ch. 8 - Axiomatic Semantics of State Machines, pages 179-204. Wiley, 2009.

31. S. Leue, A. Stefanescu, and W. Wei. An AsmL semantics for dynamic structures and run time schedulability in UML-RT. In Richard F. Paige and Bertrand Meyer, editors, Proc. of Objects, Components, Models and Patterns (TOOLS EUROPE 2008), volume 11 of Lecture Notes in Business Information Processing, pages 238-257. Springer, 2008.

32. X. Li, Z. Liu, and H. Jifeng. A formal semantics of UML sequence diagrams. In Proc. of the 2004 Australian Software Engineering Conference, pages 168 - 177, 2004.

33. J. Merseguer, S. Bernardi, J. Campos, and S. Donatelli. A Compositional Semantics for UML State Machines Aimed at Performance Evaluation. In Proceedings of the 6th International Workshop on Discrete Event Systems, pages 295 302. IEEE Computer Society Press, 2002.

34. R. Milner. A Calculus of Communicating Systems. Springer, 1980.

35. R. Milner, J. Parrow, and D. Walker. A calculus of mobile processes, parts I and II. Reports ECS-LFCS-89-85 and ECS-LFCS-89-86 86, Computer Science Dept., University of Edinburgh, March 1989.

36. M.O. Möller, A. David, and W. Yi. Verification of UML statechart with real-time extensions. In Fundamental Approaches to Software Engineering (FASE'2002), volume 2306 of LNCS, pages 218-232. Springer-Verlag, 2003.

37. R. Mrowka and T. Szmuc. UML Statecharts Compositional Semantics in LOTOS. In 2008 International Symposium on Parallel and Distributed Computing, pages 459-463. IEEE Computer Society Press, 2008. 
38. D. Muthiayen. Real-time reactive system development : a formal approach based on UML and PVS. PhD thesis, Concordia University, 2000.

39. M. Y. Ng and M. Butler. Towards Formalizing UML State Diagrams in CSP. In Proc. of SEFM'03, pages 138-147. IEEE Computer Society, 2003.

40. Object Management Group. UML Profile For Schedulability, Performance, And Time v1.1. http://www.omg.org/spec/SPTP/, January 2005.

41. Object Management Group. UML Profile For MARTE: Modeling And Analysis Of Real-Time Embedded Systems v1.1. http://www.omg.org/spec/MARTE/, June 2011.

42. Object Management Group. UML Superstructure Specification v2.4.1. http://www.omg.org/spec/UML/2.4.1/, August 2011.

43. Object Management Group. OMG Systems Modeling Language (OMG SysML ${ }^{\mathrm{TM}}$ ). http://www.omg.org/spec/SysML/1.3/, June 2012.

44. Object Management Group. UML Superstructure Specification v2.5. http://www.omg.org/spec/UML/2.5/, September 2012.

45. I. Paltor. The Semantics of UML State Machines. Technical report, 1999.

46. E. Posse. Modelling and Simulation of dynamic structure, discrete-event systems. Ph.D. Thesis, School of Computer Science. McGill University, August 2008.

47. E. Posse. A real-time extension to the $\pi$-calculus. Tech. Report 2009-557, School of Computing - Queen's University, http://www.cs.queensu.ca, 2009.

48. E. Posse. The $\pi_{k l t}$-calculus: formal definition. Tech. Report 2012-591, School of Computing - Queen's University, http://www.cs.queensu.ca, July 2012.

49. E. Posse and J. Dingel. kiltera: a language for timed, event-driven, mobile and distributed simulation. In Proc. of the 14th IEEE/ACM International Symposium on Distributed Simulation and Real Time Applications (DS-RT 2010), 2010.

50. E. Posse and J. Dingel. Theory and implementation of a real-time extension to the $\pi$-calculus. In Proc. Int. Conf. on Formal Techniques for Distributed Systems (FMOODSEFORTE'10), LNCS, 2010.

51. E. Posse and H. Vangheluwe. kiltera: A simulation language for timed, dynamic structure systems. In Proc. of the 40th Annual Simulation Symposium (ANSS'07), 2007.

52. R. Ramos, A. Sampaio, and A. Mota. A semantics for UML-RT active classes via mapping into Circus. In Proc. Int. Conf. on Formal Methods for Open Object-Based Distributed Systems FMOODS'05, volume 3535 of LNCS, pages 99-114. Springer, 2005.

53. SAE International. Architecture Analysis \& Design Language (AADL). SAE Standard AS5506b, 10 September 2012.

54. B. Selic. Using UML for modeling complex real-time systems. In Frank Mueller and Azer Bestavros, editors, Languages, Compilers, and Tools for Embedded Systems (LCTES'98), volume 1474 of LNCS, pages 250-260. Springer, 1998.

55. B. Selic. Personal Communication, 1 February 2012.

56. B. Selic, G. Gullekson, and P. T. Ward. Real-Time Object Oriented Modeling. Wiley \& Sons, 1994.

57. B. Selic and J. Rumbaugh. Using UML for modeling complex real-time systems. Whitepaper, Rational Software Corp., 1998.

58. S. Shankar and S. Asa. Formal semantics of UML with real-time constructs. In $U M L$, volume 2863 of $L N C S$, pages 60-75. Springer, 2003.
59. H. Störrle and J. H. Hausmann. Towards a formal semantics of UML 2.0 activities. In In Proceedings German Software Engineering Conference, volume 65 of LNI, pages 117-128, 2005.

60. M. von der Beeck. A structured operational semantics for UML-statecharts. SoSyM, 1(2):130-141, 2002.

61. M. von der Beeck. A formal semantics of UML-RT. In Proc. of MoDELS'06, pages 768-782, 2006.

62. W. L. Yeung, K R.P.H. Leung, J. Wang, and W. Dong. Improvements Towards Formalizing UML State Diagrams in CSP. In Proc. of APSEC-'05, pages 176 - 184. IEEE Computer Society, 2005.

63. B. P. Zeigler, H. Praehofer, and T. G. Kim. Theory of Modeling and Simulation. Academic Press, first edition, 1976.

64. B. P. Zeigler, H. Praehofer, and T. G. Kim. Theory of Modeling and Simulation. Academic Press, second edition, 2000 .

65. T. Zhang, S. Huang, and H. Huang. An Operational Semantics for UML RT-Statechart in Model Checking Context. In Proc. of the 4th Int. Conf. on Internet Computing for Science and Engineering (ICICSE), 2009, pages 12-18, 2009.

\section{Semantic Variation Points}

1. Alternative semantics could include giving priority to the states higher in the hierarchy, or to leave the choice as nondeterministic.

2. In UML 2, alternative semantics include 1) shallow history, remembering only the immediate sub-state; 2) allowing both deep and shallow history; 3) no history.

3. The action language is a major semantic variation point, but it should include at least an action to send messages. Other common actions concern operations on capsules such as accessing/modifying attributes, incarnating/destroying optional sub-capsules, or rewiring ports. IBM RSA-RTE supports three action languages: $\mathrm{C}++$, Java and UAL (UML Action Language), a Java-like language closely related to the OMG ALF standard.

4. This may be treated in a different way, and handle the event in the same way regardless of whether the state was previously visited.

5. The forwarding of events down to the active sub-state is done in order to account for the priority of inner enabled transitions over outer transitions. A different priority scheme would be changed here. For example, giving outer transitions priority would attempt the Choice process first and if no alternative was there, the Forward process would be tried instead. Allowing non-deterministic choice between transitions at different levels of nesting would require a different approach with no forwarding involved.

6. There are many possible implementations of the event pool, of which the most natural would be a priority queue, where the priority is an attribute of the event itself.

7. Alternatively this could be changed to initializing the top first and then the sub-capsules, or a more general approach allowing initialization in any order.

8. In this definition we allow only binary connection, i.e., each connector links only two ports, and ports have multiplicity 1 . To support $n$-ary multiplicity the definition of the service handler should be adapted accordingly. 